\title{
Orbit Functions
}

Anatoliy KLIMYK ${ }^{\dagger}$ and Jiri PATERA $\ddagger$

${ }^{\dagger}$ Bogolyubov Institute for Theoretical Physics, 14-b Metrologichna Str., Kyiv, 03143 Ukraine

E-mail: aklimyk@bitp.kiev.ua

$¥$ Centre de Recherches Mathématiques, Université de Montréal, C.P.6128-Centre ville, Montréal, H3C 3J\%, Québec, Canada

E-mail: patera@crm.umontreal.ca

Received January 04, 2006; Published online January 19, 2006

Original article is available at http://www.emis.de/journals/SIGMA/2006/Paper006/

\begin{abstract}
In the paper, properties of orbit functions are reviewed and further developed. Orbit functions on the Euclidean space $E_{n}$ are symmetrized exponential functions. The symmetrization is fulfilled by a Weyl group corresponding to a Coxeter-Dynkin diagram. Properties of such functions will be described. An orbit function is the contribution to an irreducible character of a compact semisimple Lie group $G$ of rank $n$ from one of its Weyl group orbits. It is shown that values of orbit functions are repeated on copies of the fundamental domain $F$ of the affine Weyl group (determined by the initial Weyl group) in the entire Euclidean space $E_{n}$. Orbit functions are solutions of the corresponding Laplace equation in $E_{n}$, satisfying the Neumann condition on the boundary of $F$. Orbit functions determine a symmetrized Fourier transform and a transform on a finite set of points.
\end{abstract}

Key words: orbit functions; Coxeter-Dynkin diagram; Weyl group; orbits; products of orbits; orbit function transform; finite orbit function transform; Neumann boundary problem; symmetric polynomials

2000 Mathematics Subject Classification: 33-02; 33E99; 42C15; 58C40

\section{Introduction}

In this paper we consider orbit functions - multivariable functions having many beautiful and useful properties and applicable both in mathematics and engineering. For this reason, they certainly can be treated as special functions [1], although there is no generally accepted definition of special functions $[2,3,4]$.

Orbit functions are closely related to finite groups $W$ of geometric symmetries generated by reflection transformations $r_{i}$ (that is, such that $r_{i}^{2}=1$ ), $i=1,2, \ldots, n$, of an $n$-dimensional Euclidean space $E_{n}$ with respect to $(n-1)$-dimensional subspaces containing the origin. Namely, we take a point $\lambda \in E_{n}$ (in a certain coordinate system) and act upon $\lambda$ using all elements of the group $W$. If $O(\lambda)$ is the orbit of the point $\lambda$, that is the set of all different points of the form $w \lambda, w \in W$, then the orbit function, determined by the point $\lambda$, coincides with

$$
\phi_{\lambda}(x)=\sum_{\mu \in O(\lambda)} e^{2 \pi \mathrm{i}\langle\mu, x\rangle}
$$

where $\langle\mu, x\rangle$ is the scalar product on $E_{n}$. Clearly, these functions are invariant with respect to the group $W: \phi_{\lambda}(w x)=\phi_{\lambda}(x), w \in W$. This is the main property of orbit functions, a property which contributes in great part to the usefulness of orbit functions in applications. An orbit function $\phi_{\lambda}(x)$ can be understood as a symmetrized (by means of the group $W$ ) exponential function. 
In this paper, we consider only orbit functions related to the symmetry groups $W$, which are Weyl groups of semisimple Lie groups (semisimple Lie algebras). Such orbit functions are closely related to irreducible representations of the corresponding semisimple compact Lie groups $G$. Namely, for $\lambda$ with integral coordinates, they are constituents of traces (characters) of finite dimensional representations of $G$. Although characters contain all (or almost all) information about the corresponding irreducible representations, they are seldom used as special functions due to the fact that a construction of characters is rather complicated, whereas orbit functions have a much simpler structure.

Orbit functions can be considered as a certain modification of monomial symmetric (Laurent) polynomials $m_{\lambda}(y)$ which are determined by the formula $m_{\lambda}(y)=\sum_{\mu \in O(\lambda)} y^{\mu}$, where $y=\left(y_{1}, y_{2}\right.$, $\left.\ldots, y_{n}\right), \mu=\left(\mu_{1}, \mu_{2}, \ldots, \mu_{n}\right)$ and $y^{\mu}=y_{1}^{\mu_{1}} \cdots y_{n}^{\mu_{n}}$. Namely, replacing $e^{2 \pi \mathrm{i} x_{i}}$ by $y_{i}, i=1,2, \ldots, n$, in the definition of an orbit function gives a corresponding monomial symmetric polynomial. It is generally accepted that monomial symmetric polynomials play a very important role in the theory of symmetric polynomials [5, 6, 7, 8] (Schur polynomials, Macdonald symmetric polynomials, Jacobi polynomials of many variables, etc.).

If we were to expand on the practical applicability of orbit functions, it would be necessary to reference works relevant to our present topic, and to consider some 'abstract' and rather challenging problems $[9,10,11,12,13,14]$.

The motivation for this article is the recent recognition of the fact that orbit functions are indispensable to the vast generalization of the discrete cosine transform [15], which has proven to be very useful in recent years [16]. In addition, it appears that it is difficult to overestimate the future role of orbit functions in some applications requiring data compression [15].

A generalization of the discrete cosine transform based on orbit functions (discrete orbit function transform) can be useful in models of quantum field theory on discrete lattices (especially when such models admit a discrete symmetry).

Our present goal is to bring together, in full generality, diverse facts about orbit functions, many of which are not found in the literature, although they often are straightforward consequences of known facts.

In general, for a given transformation group $W$ of the Euclidean space $E_{n}$, most of the properties of orbit functions described in this paper are implications of properties either of orbits of the group $W$ (for description of reflection groups see, for example, [17] and [18]), or of the irreducible characters of the corresponding compact semisimple Lie group $G$ of rank $n$ (for an exposition of the theory of finite dimensional representations of semisimple Lie groups and their characters see, for example, [19]).

The title of the article refers to the problem which was solved in [20] for an equilateral triangle, which is the fundamental domain of the affine Weyl group $W^{\text {aff }}$, corresponding to the simple Lie algebra $A_{2}$ and to the compact group $S U(3)$. Through the use of an entirely different method [21], orbit functions provide a solution to this problem for any compact simple Lie group. It was shown that orbit functions are eigenfunctions of the $n$-dimensional Laplace operator on the simplexes, which are fundamental domains of compact simple Lie groups, with the Neumann boundary value requirement (normal derivatives are zero). The eigenvalues are given explicitly.

For each transformation group $W$, the orbit functions $\phi_{\lambda}$ with integral $\lambda$ form a complete orthogonal basis in the space of symmetric (with respect to $W$ ) polynomials in $e^{2 \pi \mathrm{i} x_{j}}$ or in the Hilbert space obtained by closing this space with respect to an appropriate scalar product.

In the case where the group $W$ is a direct product of its subgroups, say $W=W_{1} \times W_{2}$, the fundamental domain is the Cartesian product of fundamental domains for $W_{1}$ and $W_{2}$. Similarly, the orbit functions of $W$ are products of orbit functions of $W_{1}$ and $W_{2}$. Hence it suffices to carry out our considerations for groups $W$ which cannot be represented as a product of its subgroups (that is, for such $W$ for which a Coxeter-Dynkin diagram is connected). 
It is generally accepted that the $n$-dimensional exponential function $e^{2 \pi \mathrm{i}\left(m_{1} x_{1}+\cdots+m_{n} x_{n}\right)}$ can be considered for integral values of $m_{1}, m_{2}, \ldots, m_{n}$. Then it is a function on a torus, and determines a decomposition into Fourier series. When the function is considered for any real values of $m_{1}, m_{2}, \ldots, m_{n}$, then it is a function on the whole space $\mathbb{R}$ and determines a decomposition into Fourier integrals of exponential functions. Orbit functions have similar properties. For integral $\lambda$ (that is, when coordinates of $\lambda$ in a certain coordinate system are integers), orbit functions are functions on a torus admitting a symmetry with respect to the Weyl group $W$. They determine a decomposition into a sum of orbit functions (symmetrized Fourier series). If we admit any real values for coordinates of $\lambda$, then they are functions on $\mathbb{R}$ (admitting a symmetry with respect to $W$ ). We then have to replace decompositions into series by decompositions into integrals of orbit functions. The first case is more interesting for applications. Nevertheless, we consider in our exposition a general case of orbit functions, with emphasis on an integral $\lambda$.

The symmetrized Fourier transform (the orbit function transform) is of importance in different branches of pure and applied mathematics in the context of theories which admit discrete symmetries. In particular, such symmetries are often encountered in theoretical and mathematical physics.

In this paper we deal only with orbit functions symmetric with respect to Weyl groups. There exist antisymmetric orbit functions [22]. They will be reviewed in a separate paper.

In this article, many examples of dimensions 2 and 3 are shown because they are likely to be used more often. Hopefully, they can be understood without the general arguments presented in this paper by directly verifying their properties through explicit calculation.

However, since our conclusions are to be general as to type of the transformation group $W$, it is imperative to profit from the uniformity of the pertinent parts of the theory of geometric symmetry groups: many general facts of the theory are recalled without explanation and with only a few references to the literature.

\section{$2 \quad$ Weyl group orbits}

\subsection{Coxeter-Dynkin diagrams and Cartan matrices}

As indicated in the Introduction, the sets of orbit functions on the $n$-dimensional Euclidean space $E_{n}$ are determined by finite transformation groups $W$, generated by reflections $r_{i}, i=1,2, \ldots, n$ (a characteristic property of reflections is the equality $r_{i}^{2}=1$ ). We are interested in groups $W$ which are Weyl groups of semisimple Lie groups (semisimple Lie algebras). Such Weyl groups, together with the corresponding systems of reflections $r_{i}, i=1,2, \ldots, n$, are determined by Coxeter-Dynkin diagrams (for the theory of such groups, see [17] and [18]). There are 4 series and 5 separate simple Lie algebras, each with a uniquely determined Weyl groups $W$. They are denoted as

$$
A_{n}(n \geq 1), \quad B_{n}(n \geq 3), \quad C_{n}(n \geq 2), \quad D_{n}(n \geq 4), \quad E_{6}, \quad E_{7}, \quad E_{8}, \quad F_{4}, \quad G_{2} .
$$

These Lie algebras correspond to connected Coxeter-Dynkin diagrams. The index below denotes a number of simple roots. This number is called a rank of the corresponding simple Lie algebra.

Semisimple Lie algebras (direct sums of simple Lie subalgebras) correspond to CoxeterDynkin diagrams, which in turn consist of connected parts corresponding to simple Lie subalgebras; these parts are not connected with each other (for a description of the correspondence between simple Lie algebras and Coxeter-Dynkin diagrams, see, for example, [19]). Thus, it is sufficient to describe the Coxeter-Dynkin diagrams that correspond to simple Lie algebras. They have the form 

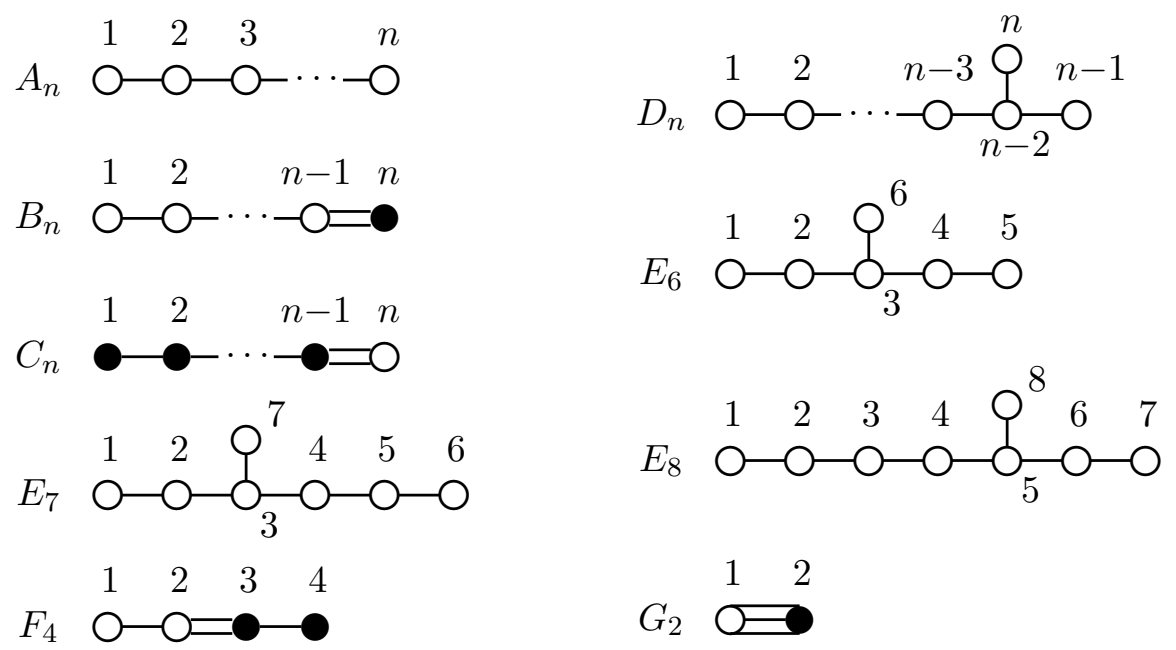

A diagram gives a certain non-orthogonal basis $\left\{\alpha_{1}, \ldots, \alpha_{n}\right\}$ in the Euclidean space $E_{n}$. Each node is associated with a basis vector $\alpha_{k}$, called a simple root. A direct link between two nodes indicates that the corresponding basis vectors are not orthogonal. Conversely, the absence of a direct link between nodes implies orthogonality of the corresponding vectors. Single, double, and triple links indicate that relative angles between the corresponding two simple roots are $2 \pi / 3,3 \pi / 4,5 \pi / 6$, respectively. There may be only two cases: all simple roots are of the same length or there are only two different lengths of simple roots. In the first case all simple roots are denoted by white nodes. In the case of two lengths, shorter roots are denoted by black nodes and longer ones by white nodes. Lengths of roots are determined uniquely up to a common constant. For the cases $B_{n}, C_{n}$, and $F_{4}$, the squared longer root length is double the squared shorter root length. For $G_{2}$, the squared longer root length is triple the squared shorter root length.

It is necessary to fix an order of simple roots. For this reason, roots on Coxeter-Dynkin diagrams are numbered.

To each Coxeter-Dynkin diagram there corresponds a Cartan matrix $M$, consisting of the entries

$$
M_{j k}=\frac{2\left\langle\alpha_{j}, \alpha_{k}\right\rangle}{\left\langle\alpha_{k}, \alpha_{k}\right\rangle}, \quad j, k \in\{1,2, \ldots, n\},
$$

where $\langle x, y\rangle$ denotes a scalar product of $x, y \in E_{n}$. All numbers $M_{i j}$ are integers. Cartan matrices of simple Lie algebras are given in many books (see, for example, [23]). We give them for the cases of ranks 2 and 3 because of their frequent usage later on:

$$
\begin{aligned}
& A_{2}:\left(\begin{array}{cc}
2 & -1 \\
-1 & 2
\end{array}\right), \quad C_{2}:\left(\begin{array}{cc}
2 & -1 \\
-2 & 2
\end{array}\right), \quad G_{2}:\left(\begin{array}{cc}
2 & -3 \\
-1 & 2
\end{array}\right), \\
& A_{3}:\left(\begin{array}{ccc}
2 & -1 & 0 \\
-1 & 2 & -1 \\
0 & -1 & 2
\end{array}\right), \quad B_{3}:\left(\begin{array}{ccc}
2 & -1 & 0 \\
-1 & 2 & -2 \\
0 & -1 & 2
\end{array}\right), \quad C_{3}:\left(\begin{array}{ccc}
2 & -1 & 0 \\
-1 & 2 & -1 \\
0 & -2 & 2
\end{array}\right) \text {. }
\end{aligned}
$$

The lengths of the basis vectors $\alpha_{i}$ are fixed by the corresponding Coxeter-Dynkin diagram up to a constant. We adopt the standard choice of Lie theory, namely

$$
\langle\alpha, \alpha\rangle=2
$$

for the longer (whenever there are two lengths) of the basis vectors. The Coxeter-Dynkin diagram differentiates long and short simple roots by node color. 


\subsection{Weyl group}

The Coxeter-Dynkin diagram uniquely determines the corresponding transformation group of $E_{n}$, generated by the reflections $r_{i}, i=1,2, \ldots, n$. Namely, the transformation $r_{i}$, corresponding to a simple root $\alpha_{i}$, is a reflection with respect to $(n-1)$-dimensional linear subspace (hyperplane) of $E_{n}$ (containing the origin) orthogonal to $\alpha_{i}$. It is well-known that such reflections are given by the formula

$$
r_{i} x=x-\frac{2\left\langle x, \alpha_{i}\right\rangle}{\left\langle\alpha_{i}, \alpha_{i}\right\rangle} \alpha_{i}, \quad i=1,2, \ldots, n, \quad x \in E_{n} .
$$

Each reflection $r_{i}$ can be thought as attached to the $i$-th node of the corresponding CoxeterDynkin diagram.

The finite group $W$, generated by the reflections $r_{i}, i=1,2, \ldots, n$, is called a Weyl group corresponding to a given Coxeter-Dynkin diagram. If a Weyl group $W$ corresponds to a CoxeterDynkin diagram of a simple Lie algebra $L$, then this Weyl group is denoted by $W(L)$. The properties of Weyl groups are well known. The orders (numbers of elements) of Weyl groups are given by the formulas

$$
\begin{aligned}
& \left|W\left(A_{n}\right)\right|=(n+1) !, \quad\left|W\left(B_{n}\right)\right|=\left|W\left(C_{n}\right)\right|=2^{n} n !, \quad\left|W\left(D_{n}\right)\right|=2^{n-1} n !, \\
& \left|W\left(E_{6}\right)\right|=51840, \quad\left|W\left(E_{7}\right)\right|=2903040, \quad\left|W\left(E_{8}\right)\right|=696729600, \\
& \left|W\left(F_{4}\right)\right|=1152, \quad\left|W\left(G_{2}\right)\right|=12 .
\end{aligned}
$$

In particular,

$$
\left|W\left(A_{2}\right)\right|=6, \quad\left|W\left(C_{2}\right)\right|=8, \quad\left|W\left(A_{3}\right)\right|=24, \quad\left|W\left(C_{3}\right)\right|=48 .
$$

\section{$2.3 \quad$ Roots and weights}

A Coxeter-Dynkin diagram determines a system of simple roots in the Euclidean space $E_{n}$. An action of elements of the Weyl group $W$ upon simple roots leads to a finite system of vectors, which is invariant with respect to $W$. A set of all these vectors is called a system of roots associated with a given Coxeter-Dynkin diagram. It is denoted by $R$. As we see, a system of roots $R$ is calculated from the simple roots by a straightforward algorithm. Root systems $R$ which correspond to Coxeter-Dynkin diagrams in 2-dimensional Euclidean spaces are shown below:

Root system of $A_{2}$ :

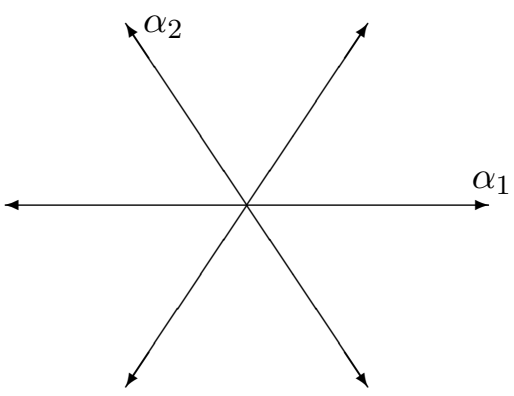


Root system of $C_{2}$ :

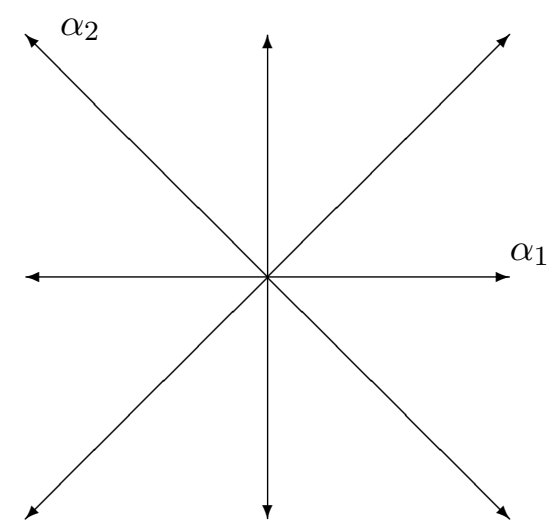

Root system of $G_{2}$ :

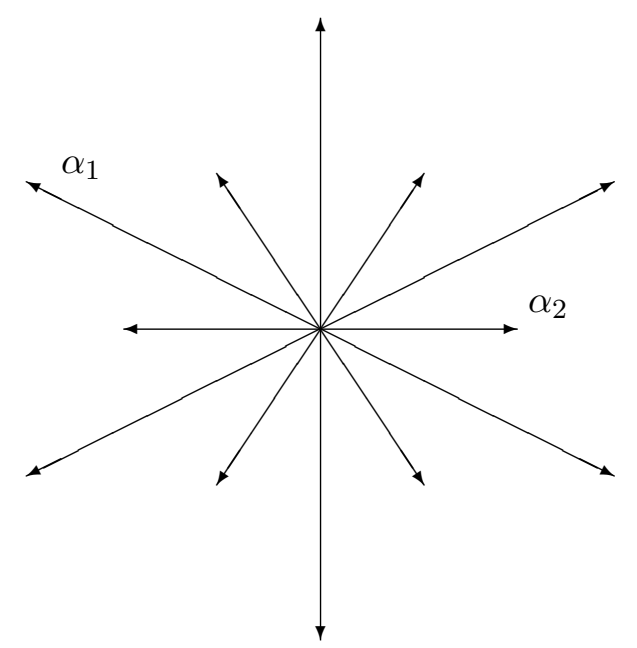

It is proven (see, for example, [19]) that roots of $R$ are linear combinations of simple roots with integral coefficients. Moreover, there exist no roots which are linear combinations of simple roots $\alpha_{i}, i=1,2, \ldots, n$, both with positive and negative coefficients. Therefore, the set of roots $R$ can be represented as a union $R=R_{+} \cup R_{-}$, where $R_{+}$(respectively $R_{-}$) is the set of roots which are linear combinations of simple roots with positive (negative) coefficients. The set $R_{+}$ (the set $R_{-}$) is called a set of positive (negative) roots. The root systems of simple Lie algebras of rank $\leq 12$ are shown, for example, in [23].

The set of all linear combinations

$$
Q=\left\{\sum_{i=1}^{n} a_{i} \alpha_{i} \mid a_{i} \in \mathbb{Z}\right\} \equiv \bigoplus_{i} \mathbb{Z} \alpha_{i}
$$

is called a root lattice corresponding to a given Coxeter-Dynkin diagram. Its subset

$$
Q_{+}=\left\{\sum_{i=1}^{n} a_{i} \alpha_{i} \mid a_{i}=0,1,2, \ldots\right\}
$$

is called a positive root lattice.

To each root $\alpha \in R$ there corresponds a coroot $\alpha^{\vee}$ defined by the formula

$$
\alpha^{\vee}=\frac{2 \alpha}{\langle\alpha, \alpha\rangle} \text {. }
$$


It is easy to see that $\alpha^{\vee \vee}=\alpha$. The set of all linear combinations

$$
Q^{\vee}=\left\{\sum_{i=1}^{n} a_{i} \alpha_{i}^{\vee} \mid a_{i} \in \mathbb{Z}\right\} \equiv \bigoplus_{i} \mathbb{Z} \alpha_{i}^{\vee}
$$

is called a coroot lattice corresponding to a given Coxeter-Dynkin diagram. The subset

$$
Q_{+}^{\vee}=\left\{\sum_{i=1}^{n} a_{i} \alpha_{i}^{\vee} \mid a_{i}=0,1,2, \ldots\right\}
$$

is called a positive coroot lattice.

As noted above, the set of simple roots $\alpha_{i}, i=1,2, \ldots, n$, is a basis of the space $E_{n}$. In addition to the $\alpha$-basis, it is convenient to introduce the $\omega$-basis, $\omega_{1}, \omega_{2}, \ldots, \omega_{n}$ (also called the basis of fundamental weights). The two bases are dual to each other in the following sense:

$$
\frac{2\left\langle\alpha_{j}, \omega_{k}\right\rangle}{\left\langle\alpha_{j}, \alpha_{j}\right\rangle} \equiv\left\langle\alpha_{j}^{\vee}, \omega_{k}\right\rangle=\delta_{j k}, \quad j, k \in\{1, \ldots, n\} .
$$

The $\omega$-basis (as the $\alpha$-basis) is not orthogonal.

Note that the factor $2 /\left\langle\alpha_{j}, \alpha_{j}\right\rangle$ can take only three values. Indeed, with the standard normalization of root lengths, we have

$$
\begin{array}{ll}
\frac{2}{\left\langle\alpha_{k}, \alpha_{k}\right\rangle}=1 \quad \text { for roots of } A_{n}, D_{n}, E_{6}, E_{7}, E_{8}, \\
\frac{2}{\left\langle\alpha_{k}, \alpha_{k}\right\rangle}=1 \quad \text { for long roots of } B_{n}, C_{n}, F_{4}, G_{2}, \\
\frac{2}{\left\langle\alpha_{k}, \alpha_{k}\right\rangle}=2 \quad \text { for short roots of } B_{n}, C_{n}, F_{4}, \\
\frac{2}{\left\langle\alpha_{k}, \alpha_{k}\right\rangle}=3 \quad \text { for short root of } G_{2} .
\end{array}
$$

The $\alpha$ - and $\omega$-bases are related by the Cartan matrix (2.1) and by its inverse:

$$
\alpha_{j}=\sum_{k=1}^{n} M_{j k} \omega_{k}, \quad \omega_{j}=\sum_{k=1}^{n}\left(M^{-1}\right)_{j k} \alpha_{k} .
$$

For ranks 2 and 3 the inverse Cartan matrices are of the form

$$
\begin{aligned}
& A_{2}: \frac{1}{3}\left(\begin{array}{cc}
2 & 1 \\
1 & 2
\end{array}\right), \quad C_{2}:\left(\begin{array}{cc}
1 & 1 / 2 \\
1 & 1
\end{array}\right), \quad G_{2}:\left(\begin{array}{cc}
2 & 3 \\
1 & 2
\end{array}\right), \\
& A_{3}: \frac{1}{4}\left(\begin{array}{lll}
3 & 2 & 1 \\
2 & 4 & 2 \\
1 & 2 & 3
\end{array}\right), \quad B_{3}: \frac{1}{2}\left(\begin{array}{lll}
2 & 2 & 2 \\
2 & 4 & 4 \\
1 & 2 & 3
\end{array}\right), \quad C_{3}: \frac{1}{2}\left(\begin{array}{lll}
2 & 2 & 1 \\
2 & 4 & 2 \\
2 & 4 & 3
\end{array}\right) .
\end{aligned}
$$

Later on we will need to calculate the scalar product $\langle x, y\rangle$ where $x$ and $y$ are given by coordinates $x_{i}$ and $y_{i}$ relative to the $\omega$-basis. It is given by the formula

$$
\langle x, y\rangle=\frac{1}{2} \sum_{j, k=1}^{n} x_{j} y_{k}\left(M^{-1}\right)_{j k}\left\langle\alpha_{k} \mid \alpha_{k}\right\rangle=x M^{-1} D y^{T} \equiv x S y^{T},
$$

where $D$ is the diagonal matrix $\operatorname{diag}\left(\frac{1}{2}\left\langle\alpha_{1} \mid \alpha_{1}\right\rangle, \ldots, \frac{1}{2}\left\langle\alpha_{n} \mid \alpha_{n}\right\rangle\right)$. Matrices $S$, called 'quadratic form matrices', are shown in [23] for all connected Coxeter-Dynkin diagrams. 
The sets $P$ and $P_{+}$, defined as

$$
P=\mathbb{Z} \omega_{1}+\cdots+\mathbb{Z} \omega_{n} \supset P_{+}=\mathbb{Z}^{\geq 0} \omega_{1}+\cdots+\mathbb{Z}^{\geq 0} \omega_{n},
$$

are respectively called the weight lattice and the cone of dominant weights. The set $P$ can be characterized as a set of all $\lambda \in E_{n}$ such that $2\left\langle\lambda, \alpha_{j}\right\rangle /\left\langle\alpha_{j}, \alpha_{j}\right\rangle \equiv\left\langle\lambda, \alpha_{j}^{\vee}\right\rangle \in \mathbb{Z}$ for all simple roots $\alpha_{j}$. Clearly, $Q \subset P$. Moreover, the additive group $P / Q$ is finite.

The smallest non-vanishing dominant weights $\omega_{k}, k=1,2, \ldots, n$, defined by (2.4), are called fundamental weights. Throughout this article we will often use the following notation for weights in the $\omega$-basis:

$$
\lambda=\sum_{j=1}^{n} a_{j} \omega_{j}=\left(\begin{array}{llll}
a_{1} & a_{2} & \cdots & a_{n}
\end{array}\right) .
$$

If $x=\sum_{j=1}^{n} b_{j} \alpha_{j}^{\vee}$, then

$$
\langle\lambda, x\rangle=\sum_{j=1}^{n} a_{j} b_{j} .
$$

There is a unique highest (long) root $\xi$ and a unique highest short root $\xi_{s}$. A highest root can be written in the form

$$
\xi=\sum_{i=1}^{n} m_{i} \alpha_{i}=\sum_{i=1}^{n} m_{i} \frac{\left\langle\alpha_{i}, \alpha_{i}\right\rangle}{2} \alpha_{i}^{\vee} \equiv \sum_{i=1}^{n} q_{i} \alpha_{i}^{\vee} .
$$

Coefficients $m_{i}$ and $q_{i}$ can be considered as attached to the $i$-th node of the Coxeter-Dynkin diagram. They are called marks and comarks. They are often listed in the literature (see, for example, [23] and [24]). In root systems with two lengths of roots, namely in $B_{n}, C_{n}, F_{4}$ and $G_{2}$, the highest (long) root $\xi$ is the following:

$$
\begin{aligned}
& B_{n}: \xi=(010 \cdots 0)=\alpha_{1}+2 \alpha_{2}+2 \alpha_{3}+\cdots+2 \alpha_{n}, \\
& C_{n}: \xi=(20 \cdots 0)=2 \alpha_{1}+2 \alpha_{2}+\cdots+2 \alpha_{n-1}+\alpha_{n} \text {, } \\
& F_{4}: \xi=(1000)=2 \alpha_{1}+3 \alpha_{2}+4 \alpha_{3}+2 \alpha_{4} \text {, } \\
& G_{2}: \xi=\quad(10)=2 \alpha_{1}+3 \alpha_{2} \text {. }
\end{aligned}
$$

In case of $A_{n}, D_{n}$ and $E_{n}$, all roots are of the same length and we have

$$
\begin{aligned}
& A_{n}: \xi=(10 \cdots 01)=\alpha_{1}+\cdots+\alpha_{n}, \\
& D_{n}: \xi=(010 \cdots 0)=\alpha_{1}+2 \alpha_{2}+\cdots+2 \alpha_{n-2}+\alpha_{n-1}+\alpha_{n}, \\
& E_{6}: \xi=(00 \cdots 01)=\alpha_{1}+2 \alpha_{2}+3 \alpha_{3}+2 \alpha_{4}+\alpha_{5}+2 \alpha_{6} \text {, } \\
& E_{7}: \xi=(100 \cdots 0)=2 \alpha_{1}+3 \alpha_{2}+4 \alpha_{3}+3 \alpha_{4}+2 \alpha_{5}+\alpha_{6}+2 \alpha_{7} \text {, } \\
& E_{8}: \xi=(100 \cdots 0)=2 \alpha_{1}+3 \alpha_{2}+4 \alpha_{3}+5 \alpha_{4}+6 \alpha_{5}+4 \alpha_{6}+2 \alpha_{7}+3 \alpha_{8} \text {. }
\end{aligned}
$$

Note that for highest root $\xi$ we have

$$
\xi^{\vee}=\xi .
$$

Moreover, if all simple roots are of the same length, then

$$
\alpha_{i}^{\vee}=\alpha_{i} .
$$


For this reason,

$$
\left(q_{1}, q_{2}, \ldots, q_{n}\right)=\left(m_{1}, m_{2}, \ldots, m_{n}\right)
$$

for $A_{n}, D_{n}$ and $E_{n}$. Formulas (2.13)-(2.18) determine these numbers. For short roots $\alpha_{i}$ of $B_{n}$, $C_{n}$ and $F_{4}$ we have $\alpha_{i}^{\vee}=2 \alpha_{i}$. For short root $\alpha_{2}$ of $G_{2}$ we have $\alpha_{2}^{\vee}=3 \alpha_{2}$. For this reason,

$$
\begin{array}{ll}
\left(q_{1}, q_{2}, \ldots, q_{n}\right)=(1,2, \ldots, 2,1) \quad \text { for } & B_{n}, \\
\left(q_{1}, q_{2}, \ldots, q_{n}\right)=(1,1, \ldots, 1,1) \quad \text { for } \quad C_{n} \\
\left(q_{1}, q_{2}, q_{3}, q_{4}\right)=(2,3,2,1) \quad \text { for } & F_{4}, \\
\left(q_{1}, q_{2}\right)=(2,1) \quad \text { for } G_{2} . &
\end{array}
$$

With the help of the highest root $\xi$, it is possible to construct an extended root system (which is also called an affine root system). Namely, if $\alpha_{1}, \alpha_{2}, \ldots, \alpha_{n}$ is a set of all simple roots, then the roots

$$
\alpha_{0}:=-\xi, \alpha_{1}, \alpha_{2}, \ldots, \alpha_{n}
$$

constitute a set of simple roots of the corresponding extended root system. Taking into account the orthogonality (non-orthogonality) of the root $\alpha_{0}$ to other simple roots, the diagram of an extended root system can be constructed (which is an extension of the corresponding CoxeterDynkin diagram; see, for example, [24]). Note that for all simple Lie algebras (except for $A_{n}$ ) only one simple root is orthogonal to the root $\alpha_{0}$. In the case of $A_{n}$, the two simple roots $\alpha_{1}$ and $\alpha_{n}$ are not orthogonal to $\alpha_{0}$.

\subsection{Weyl group orbits}

The $(n-1)$-dimensional linear subspaces of $E_{n}$, orthogonal to positive roots and containing the origin, divide the space $E_{n}$ into connected parts, which are called Weyl chambers. The number of such chambers is equal to the order of the corresponding Weyl group $W$. Elements of the Weyl group $W$ permute these chambers. There exists a single chamber $D_{+}$such that

$$
\left\langle\alpha_{i}, x\right\rangle \geq 0, \quad x \in D_{+}, \quad i=1,2, \ldots, n .
$$

It is called the dominant Weyl chamber.

Clearly, the cone of dominant weights $P_{+}$belongs to the dominant Weyl chamber $D_{+}$. (Note that it is not the case for the set $Q_{+}$.) Moreover, $P \cap D_{+}=P_{+}$.

Let $y$ be an arbitrary element of the Euclidean space $E_{n}$. We act upon $y$ by all elements of the Weyl group $W$, thus creating the set of elements $w y, w \in W$. There may exist coinciding elements in this set. We extract from this set all distinct points (we denote them by $\left.y_{1}, y_{2}, \ldots, y_{m}\right)$. The set of these points is called an orbit of $y$ with respect to the Weyl group $W$ (or a Weyl group orbit that contains $y$ ). Since $W y_{i}$ is the same as $W y_{j}$ for all $i, j=1,2, \ldots, m$, the orbit of $y$ is thus the same as the orbit of any other point $y_{i}$.

The orbit of a point $y \in E_{n}$ is denoted by $O(y)$ or $O_{W}(y)$. The number of elements contained in an orbit $O(y)$ is referred to as its size, and is denoted $|O(y)|$. One has two extremal values of the orbit size

$$
|O(0)|=1, \quad|O(y)|=|W|,
$$

where 0 is the origin of $E_{n}$ and $y$ is an intrinsic point of any Weyl chamber (that is, it is invariant only by the trivial transformation from the Weyl group $W$ ). 
Each Weyl chamber contains only one point from any given orbit $Q(y)$. In particular, in an orbit there exists only one point in the dominant Weyl chamber $D_{+}$. An orbit is usually denoted by this particular point in $D_{+}$, that is, the notation $O(y)$ means that $y \in D_{+}$.

Let $W_{y}$ be a subgroup of $W$, consisting of all elements $w \in W$, such that $w y=y$. It is called the stabilizer of the point $y$. We have

$$
|O(y)|=\frac{|W|}{\left|W_{y}\right|} .
$$

If $y \in D_{+}$, then $W_{y}$ is the subgroup generated by all reflections $r_{i}$, which correspond to the simple roots $\alpha_{i}$, such that $r_{i} y=y$.

\subsection{Orbits of $A_{1}, A_{1} \times A_{1}, A_{2}, B_{2}, G_{2}$}

There is always an orbit of size 1 , consisting of the single weight zero. We consider all the other orbits. Assuming $(a, b) \neq(0,0), a \geq 0, b \geq 0$, here is a list of the remaining orbits and their contents in the $\omega$-basis:

$$
\begin{aligned}
& A_{1}: \quad O(a) \ni(a),(-a) \\
& A_{1} \times A_{1}: \quad O(a 0) \ni(a 0),(-a 0), \quad O\left(\begin{array}{ll}
0 & b
\end{array}\right) \ni\left(\begin{array}{ll}
0 & b
\end{array}\right),(0-b), \\
& O(a b) \ni(a b),(-a b),(a-b),(-a-b) \\
& A_{2}: \quad O(a 0) \ni\left(\begin{array}{ll}
a & 0
\end{array}\right),(-a a),(0-a) \text {, } \\
& O\left(\begin{array}{ll}
0 & b
\end{array}\right) \ni\left(\begin{array}{ll}
0 & b
\end{array}\right),(b-b),(-b 0), \\
& O(a b) \ni(a b),(-a a+b),(a+b-b) \text {, } \\
& (-b-a),(-a-b a),(b-a-b) .
\end{aligned}
$$

In the cases of $C_{2}$ and $G_{2}$ (where the second simple root is the longer one for $C_{2}$ and the shorter one for $G_{2}$ ) we have

$$
\begin{aligned}
C_{2}: & O(a \quad 0) \ni \pm(a 0), \pm(-a a), \quad O(0 b) \ni \pm(0 b), \pm(2 b-b) \\
& O(a b) \ni \pm(a b), \pm(-a a+b), \pm(a+2 b-b), \pm(a+2 b-a-b), \\
G_{2}: & O\left(\begin{array}{ll}
a & 0
\end{array}\right) \ni \pm(a 0), \pm(-a 3 a), \pm(2 a-3 a), \\
& O(0 b) \ni \pm(0 b), \pm(b-b), \pm(-b 2 b), \\
& O(a b) \ni \pm(a b), \pm(-a 3 a+b), \pm(a+b-b), \\
& \pm(2 a+b-3 a-b), \pm(-a-b 3 a+2 b), \pm(-2 a-b 3 a+2 b) .
\end{aligned}
$$

\subsection{Geometric interpretation of orbits}

The elements of $O(y)$ can be interpreted as vertices of a polytope generated by reflections, starting from a single point, in $n$ mirrors intersecting at the origin. For a general method of describing such polytopes and their faces of all dimensions in $\mathbb{R}^{n}$ see [25]. For example, orbits in 2 dimensions can be interpreted as planar polygons.

In particular, the non-zero roots of Lie algebra $A_{2}$ form the orbit $O(11)$, which corresponds to the vertices of the regular hexagon. Roots of all other rank 2 Lie algebras belong to the union of two different orbits. Namely, $O(20) \cup O(02)$ in the case of $A_{1} \times A_{1}$; for $C_{2}$ it is the orbit of the long roots $O(20)$ and the orbit of the short roots $O(01)$. The long roots of $G_{2}$ form the hexagon $O(10)$, while the short roots form the hexagon $O(01)$.

The $W$-orbits in 3 dimensions include many common polytopes ([25] and [26]) with the notable exceptions of the icosahedral polytopes: icosahedron, dodecahedron, buckyball ('soccerball'), and others. 


\section{Lattices, Weyl groups and orbits for $A_{n}, B_{n}, C_{n}, D_{n}$}

In the case of Coxeter-Dynkin diagrams $A_{n-1}, B_{n}, C_{n}, D_{n}$, root and weight lattices, Weyl groups and orbits are simply described using an orthogonal coordinate system in $E_{n}$. Let us present these coordinate systems.

\subsection{The case of $A_{n}$}

For this case it is convenient to describe root and weight lattices, Weyl group and orbits in the subspace of the Euclidean space $E_{n+1}$, given by the equation

$$
x_{1}+x_{2}+\cdots+x_{n+1}=0,
$$

where $x_{1}, x_{2}, \ldots, x_{n+1}$ are the orthogonal coordinates of a point $x \in E_{n+1}$. The unit vectors in directions of these coordinates are denoted by $\boldsymbol{e}_{j}$, respectively. Clearly, $\boldsymbol{e}_{i} \perp \boldsymbol{e}_{j}, i \neq j$. The set of roots is given by the vectors

$$
\alpha_{i j}=e_{i}-e_{j}, \quad i \neq j
$$

The roots

$$
\alpha_{i j}=\boldsymbol{e}_{i}-\boldsymbol{e}_{j}, \quad i<j,
$$

are positive and the roots

$$
\alpha_{i} \equiv \alpha_{i, i+1}=\boldsymbol{e}_{i}-\boldsymbol{e}_{i+1}, \quad i=1,2, \ldots, n,
$$

constitute the system of simple roots.

If $x=\sum_{i=1}^{n+1} x_{i} \boldsymbol{e}_{i}, x_{1}+x_{2}+\cdots+x_{n+1}=0$, is a point of $E_{n+1}$, then it belongs to the dominant Weyl chamber $D_{+}$if and only if

$$
x_{1} \geq x_{2} \geq \cdots \geq x_{n+1}
$$

Indeed, if this condition is fulfilled, then $\left\langle x, \alpha_{i}\right\rangle=x_{i}-x_{i+1} \geq 0, i=1,2, \ldots, n$, and $x$ is dominant. Conversely, if $x$ is dominant, then $\left\langle x, \alpha_{i}\right\rangle \geq 0$, thus fulfilling the prescribed condition.

If $\lambda=\sum_{i=1}^{n} \lambda_{i} \omega_{i}$, then the coordinates $\lambda_{i}$ in the $\omega$-basis are connected with the orthogonal coordinates $m_{j}$ of $\lambda=\sum_{i=1}^{n+1} m_{i} \mathbf{e}_{i}$ by the formulas

$$
\begin{aligned}
& m_{1}=\frac{n}{n+1} \lambda_{1}+\frac{n-1}{n+1} \lambda_{2}+\frac{n-2}{n+1} \lambda_{3}+\cdots+\frac{2}{n+1} \lambda_{n-1}+\frac{1}{n+1} \lambda_{n}, \\
& m_{2}=-\frac{1}{n+1} \lambda_{1}+\frac{n-1}{n+1} \lambda_{2}+\frac{n-2}{n+1} \lambda_{3}+\cdots+\frac{2}{n+1} \lambda_{n-1}+\frac{1}{n+1} \lambda_{n}, \\
& m_{3}=-\frac{1}{n+1} \lambda_{1}-\frac{2}{n+1} \lambda_{2}+\frac{n-2}{n+1} \lambda_{3}+\cdots+\frac{2}{n+1} \lambda_{n-1}+\frac{1}{n+1} \lambda_{n}, \\
& \begin{array}{lllll}
\ldots & \ldots & \ldots & \ldots & \ldots
\end{array} \\
& m_{n}=-\frac{1}{n+1} \lambda_{1}-\frac{2}{n+1} \lambda_{2}-\frac{3}{n+1} \lambda_{3}-\cdots-\frac{n-1}{n+1} \lambda_{n-1}+\frac{1}{n+1} \lambda_{n}, \\
& m_{n+1}=-\frac{1}{n+1} \lambda_{1}-\frac{2}{n+1} \lambda_{2}-\frac{3}{n+1} \lambda_{3}-\cdots-\frac{n-1}{n+1} \lambda_{n-1}-\frac{n}{n+1} \lambda_{n} .
\end{aligned}
$$


The inverse formulas are

$$
\lambda_{i}=m_{i}-m_{i+1}, \quad i=1,2, \ldots, n .
$$

By means of the formula

$$
r_{\alpha} \lambda=\lambda-\frac{2\langle\lambda, \alpha\rangle}{\langle\alpha, \alpha\rangle} \alpha
$$

for the reflection respective to the hyperplane, orthogonal to a root $\alpha$, we can find that the reflection $r_{\alpha_{i j}}$ acts upon the vector $\lambda=\sum_{i=1}^{n+1} m_{i} \boldsymbol{e}_{i}$, given by orthogonal coordinates, by permuting the coordinates $m_{i}$ and $m_{j}$. Indeed, when $i>j$ we have

$$
\begin{aligned}
\lambda-\frac{2\left\langle\lambda, \alpha_{i j}\right\rangle}{\left\langle\alpha_{i j}, \alpha_{i j}\right\rangle} \alpha_{i j} & =\sum_{s=1}^{n+1} m_{s} \boldsymbol{e}_{s}-\left(m_{i}-m_{j}\right) \boldsymbol{e}_{i}+\left(m_{i}-m_{j}\right) \boldsymbol{e}_{j} \\
& =m_{1} \boldsymbol{e}_{1}+\cdots+m_{j} \boldsymbol{e}_{i}+\cdots+m_{i} \boldsymbol{e}_{j}+\cdots+m_{n+1} \boldsymbol{e}_{n+1} .
\end{aligned}
$$

Thus, $W\left(A_{n}\right)$ consists of all permutations of the orthogonal coordinates $m_{1}, m_{2}, \ldots, m_{n+1}$ of a point $\lambda$, that is, $W\left(A_{n}\right)$ coincides with the symmetric group $S_{n+1}$.

Sometimes (for example, if we wish the coordinates to be integers or non-negative integers), it is convenient to introduce the orthogonal coordinates $x_{1}, x_{2}, \ldots, x_{n+1}$ for $A_{n}$ in such a way that

$$
x_{1}+x_{2}+\cdots+x_{n+1}=m,
$$

where $m$ is some fixed real number. They are obtained from the previous orthogonal coordinates by adding the same number $m /(n+1)$ to each coordinate. Then, as one can see from (3.1), $\omega$-coordinates $\lambda_{i}=x_{i}-x_{i+1}$ and the Weyl group $W$ remain unchanged.

The orbit $O(\lambda), \lambda=\left(m_{1}, m_{2}, \ldots, m_{n+1}\right), m_{1} \geq m_{2} \geq \cdots \geq m_{n+1}$, consists of all different points

$$
\left(m_{i_{1}}, m_{i_{2}}, \ldots, m_{i_{n+1}}\right)
$$

obtained from $\left(m_{1}, m_{2}, \ldots, m_{n+1}\right)$ by permutations. The subgroup $W_{\lambda}$ of $W$ is a product of subgroups, consisting of permutations of collections of coinciding $m$ 's. For example, the orbit $O(m, 0, \ldots, 0)$ (with $n+1$ orthogonal coordinates) consists of $n+1$ points $(0, \ldots, 0, m, 0, \ldots, 0)$, where $m$ is in $j$-th place, $j=1,2, \ldots, n+1$. The subgroup $W_{\lambda}$ in this case is isomorphic to the symmetric group $S_{n}$.

The orbit $O\left(m_{1}, m_{2}, 0, \ldots, 0\right), m_{1} \neq m_{2}$, consists of $n(n+1)$ points of the form

$$
\left(0, \ldots, 0, m^{\prime}, 0, \ldots, 0, m^{\prime \prime}, 0, \ldots, 0\right)
$$

where $\left(m^{\prime}, m^{\prime \prime}\right)=\left(m_{1}, m_{2}\right)$ or $\left(m^{\prime}, m^{\prime \prime}\right)=\left(m_{2}, m_{1}\right)$. The subgroup $W_{\lambda}$ is isomorphic to the symmetric group $S_{n-1}$. If $m_{1}=m_{2}$, then this orbit consists of $n(n+1) / 2$ elements and the subgroup $W_{\lambda}$ is isomorphic to $S_{n-1} \times S_{2}$.

If we have an orbit with points given in orthogonal coordinates, it is easy, by using formula (3.1), to express those points in $\omega$-coordinates. 


\subsection{The case of $B_{n}$}

Orthogonal coordinates of a point $x \in E_{n}$ are denoted by $x_{1}, x_{2}, \ldots, x_{n}$. We denote by $\boldsymbol{e}_{i}$ the corresponding unit vectors in directions of these coordinates. Then the set of roots of $B_{n}$ is given by the vectors

$$
\alpha_{ \pm i, \pm j}= \pm \boldsymbol{e}_{i} \pm \boldsymbol{e}_{j}, \quad i \neq j, \quad \alpha_{ \pm i}= \pm \boldsymbol{e}_{i}, \quad i=1,2, \ldots, n
$$

(all combinations of signs must be taken). The roots

$$
\alpha_{i, \pm j}=\boldsymbol{e}_{i} \pm \boldsymbol{e}_{j}, \quad i<j, \quad \alpha_{i}=\boldsymbol{e}_{i}, \quad i=1,2, \ldots, n,
$$

are positive and the $n$ roots

$$
\alpha_{i}:=\boldsymbol{e}_{i}-\boldsymbol{e}_{i+1}, \quad i=1,2, \ldots, n-1, \quad \alpha_{n}=\boldsymbol{e}_{n}
$$

constitute the system of simple roots.

It is easy to see that if $\lambda=\sum_{i=1}^{n} m_{i} \boldsymbol{e}_{i}$ is a point of $E_{n}$, then this point belongs to the dominant Weyl chamber $D_{+}$if and only if

$$
m_{1} \geq m_{2} \geq \cdots \geq m_{n} \geq 0 \text {. }
$$

If $\lambda=\sum_{i=1}^{n} \lambda_{i} \omega_{i}$, then the coordinates $\lambda_{i}$ in the $\omega$-basis are connected with the orthogonal coordinates $m_{j}$ of $\lambda=\sum_{i=1}^{n} m_{i} \boldsymbol{e}_{i}$ by the formulas

$$
\begin{aligned}
& m_{1}=\lambda_{1}+\lambda_{2}+\cdots+\lambda_{n-1}+\frac{1}{2} \lambda_{n}, \\
& m_{2}=\lambda_{2}+\cdots+\lambda_{n-1}+\frac{1}{2} \lambda_{n}, \\
& \begin{array}{lllllll}
\ldots & \ldots & \ldots & \ldots & \ldots & \ldots
\end{array} \\
& m_{n}=\quad \frac{1}{2} \lambda_{n},
\end{aligned}
$$

The inverse formulas are

$$
\lambda_{i}=m_{i}-m_{i+1}, \quad i=1,2, \ldots, n-1, \quad \lambda_{n}=2 m_{n} .
$$

By means of the formula (3.2) we find that the reflection $r_{\alpha}$ acts upon orthogonal coordinates of the vector $\lambda=\sum_{i=1}^{n} m_{i} \boldsymbol{e}_{i}$ by permuting the $i$-th and $j$-th coordinates if $\alpha= \pm\left(\boldsymbol{e}_{i}-\boldsymbol{e}_{j}\right)$, as the permutation of the $i$-th and $j$-th coordinates and a change of their signs if $\alpha= \pm\left(\boldsymbol{e}_{i}+\boldsymbol{e}_{j}\right)$, and as a change of a sign of $i$-th coordinate if $\alpha= \pm \boldsymbol{e}_{i}$. Thus, the Weyl group $W\left(B_{n}\right)$ consists of all permutations of the orthogonal coordinates $m_{1}, m_{2}, \ldots, m_{n}$ of a point $\lambda$ with possible sign alternations for some of them.

The orbit $O(\lambda), \lambda=\left(m_{1}, m_{2}, \ldots, m_{n}\right), m_{1} \geq m_{2} \geq \cdots \geq m_{n} \geq 0$, consists of all different points

$$
\left( \pm m_{i_{1}}, \pm m_{i_{2}}, \ldots, \pm m_{i_{n+1}}\right)
$$

(each combination of signs is possible) obtained from $\left(m_{1}, m_{2}, \ldots, m_{n}\right)$ by permutations and alternations of signs. The subgroup $W_{\lambda}$ of $W$ is a product of the Weyl group $W\left(B_{r}\right)$, where $r$ is the number of 0 's in the weight $\left(m_{1}, m_{2}, \ldots, m_{n}\right)$, and of the subgroups, consisting of permutations of collections of coinciding non-vanishing $m$ 's. For example, the orbit $O(m, 0, \ldots, 0)$, 
$m \neq 0$, consists of $2 n$ points $(0, \ldots, 0, \pm m, 0, \ldots, 0)$, where $\pm m$ is on $j$-th place, $j=1,2, \ldots, n$. The subgroup $W_{\lambda}$ in this case is isomorphic to the Weyl group $W\left(B_{n-1}\right)$.

The orbit $O\left(m_{1}, m_{2}, 0, \ldots, 0\right), m_{1} \neq m_{2}$, consists of $4 n(n-1)$ points of the form

$$
\left(0, \ldots, 0, \pm m^{\prime}, 0, \ldots, 0, \pm m^{\prime \prime}, 0, \ldots, 0\right),
$$

where $\left(m^{\prime}, m^{\prime \prime}\right)=\left(m_{1}, m_{2}\right)$ or $\left(m^{\prime}, m^{\prime \prime}\right)=\left(m_{2}, m_{1}\right)$. The subgroup $W_{\lambda}$ is isomorphic to the Weyl group $W\left(B_{n-2}\right)$. If $m_{1}=m_{2}$, then this orbit consists of $2 n(n-1)$ elements.

If we have an orbit with points given in orthogonal coordinates, it is easy, by using formula (3.3), to transform it to $\omega$-coordinates.

\subsection{The case of $C_{n}$}

In the orthogonal coordinate system of the Euclidean space $E_{n}$ the set of roots of $C_{n}$ is given by the vectors

$$
\alpha_{ \pm i, \pm j}= \pm \boldsymbol{e}_{i} \pm \boldsymbol{e}_{j}, \quad i \neq j, \quad \alpha_{ \pm i}= \pm 2 \boldsymbol{e}_{i}, \quad i=1,2, \ldots, n,
$$

where $\boldsymbol{e}_{i}$ is the unit vector in the direction of the $i$-th coordinate $x_{i}$ (all combinations of signs must be taken into account). The roots

$$
\alpha_{i, \pm j}=e_{i} \pm e_{j}, \quad i<j, \quad \alpha_{i}=2 e_{i}, \quad i=1,2, \ldots, n,
$$

are positive and the $n$ roots

$$
\alpha_{i}:=\boldsymbol{e}_{i}-\boldsymbol{e}_{i+1}, \quad i=1,2, \ldots, n-1, \quad \alpha_{n}=2 \boldsymbol{e}_{n}
$$

constitute the system of simple roots.

It is easy to see that if $\lambda=\sum_{i=1}^{n} m_{i} \boldsymbol{e}_{i}$ is a point of $E_{n}$, then it belongs to the dominant Weyl chamber $D_{+}$if and only if

$$
m_{1} \geq m_{2} \geq \cdots \geq m_{n} \geq 0 \text {. }
$$

If $\lambda=\sum_{i=1}^{n} \lambda_{i} \omega_{i}$, then the coordinates $\lambda_{i}$ in the $\omega$-basis are connected with the coordinates $m_{j}$ of $\lambda=\sum_{i=1}^{n} m_{i} \boldsymbol{e}_{i}$ by the formulas

$$
\begin{aligned}
& m_{1}=\lambda_{1}+\lambda_{2}+\cdots+\lambda_{n-1}+\lambda_{n}, \\
& m_{2}=\lambda_{2}+\cdots+\lambda_{n-1}+\lambda_{n}, \\
& \begin{array}{llllll} 
& \ldots & \ldots & \ldots & \ldots & \ldots
\end{array} \\
& m_{n}=\quad \lambda_{n} .
\end{aligned}
$$

The inverse formulas are

$$
\lambda_{i}=m_{i}-m_{i+1}, \quad i=1,2, \ldots, n-1, \quad \lambda_{n}=m_{n} .
$$

By means of the formula (3.2) we find that the reflection $r_{\alpha}$ acts upon orthogonal coordinates of the vector $\lambda=\sum_{i=1}^{n} m_{i} \boldsymbol{e}_{i}$ by permuting the $i$-th and $j$-th coordinates if $\alpha= \pm\left(\boldsymbol{e}_{i}-\boldsymbol{e}_{j}\right)$, as the permutation of $i$-th and $j$-th coordinates and a change of their signs if $\alpha= \pm\left(\boldsymbol{e}_{i}+\boldsymbol{e}_{j}\right)$, and as a change of a sign of the $i$-th coordinate if $\alpha= \pm 2 \boldsymbol{e}_{i}$. Thus, the Weyl group $W\left(C_{n}\right)$ consists of all 
permutations of the orthogonal coordinates $m_{1}, m_{2}, \ldots, m_{n}$ of a point $\lambda$ with sign alternations for some of them, that is, this Weyl group is isomorphic to the Weyl group $W\left(B_{n}\right)$.

The orbit $O(\lambda), \lambda=\left(m_{1}, m_{2}, \ldots, m_{n}\right), m_{1} \geq m_{2} \geq \cdots \geq m_{n} \geq 0$, consists of all different points

$$
\left( \pm m_{i_{1}}, \pm m_{i_{2}}, \ldots, \pm m_{i_{n+1}}\right)
$$

(each combination of signs is possible) obtained from $\left(m_{1}, m_{2}, \ldots, m_{n}\right)$ by permutations and alternations of signs. The subgroup $W_{\lambda}$ of $W$ is a product of the Weyl group $W\left(C_{r}\right)$, where $r$ is the number of 0 's in the weight $\left(m_{1}, m_{2}, \ldots, m_{n}\right)$, and of the subgroups consisting of permutations of collections of coinciding non-vanishing $m$ 's. For example, the orbit $O(m, 0, \ldots, 0)$ consists of $2 n$ points $(0, \ldots, 0, \pm m, 0, \ldots, 0)$, where $\pm m$ is in the $j$-th place, $j=1,2, \ldots, n$. The subgroup $W_{\lambda}$ is isomorphic to the Weyl group $W\left(C_{n-1}\right)$.

The orbit $O\left(m_{1}, m_{2}, 0, \ldots, 0\right), m_{1} \neq m_{2}$, consists of $4 n(n-1)$ points of the same form as in the case of $B_{n}$. The subgroup $W_{\lambda}$ is isomorphic to the Weyl group $W\left(C_{n-2}\right)$. If $m_{1}=m_{2}$, then this orbit consists of $2 n(n-1)$ elements.

If we have an orbit with points given in orthogonal coordinates, it is easy, by using formula (3.4), to transform it to $\omega$-coordinates.

\subsection{The case of $D_{n}$}

In the orthogonal coordinate system of the Euclidean space $E_{n}$ the set of roots of $D_{n}$ is given by the vectors

$$
\alpha_{ \pm i, \pm j}= \pm \boldsymbol{e}_{i} \pm \boldsymbol{e}_{j}, \quad i \neq j,
$$

where $\boldsymbol{e}_{i}$ is the unit vector in the direction of the $i$-th coordinate (all combinations of signs must be taken into account). The roots

$$
\alpha_{i, \pm j}=\boldsymbol{e}_{i} \pm \boldsymbol{e}_{j}, \quad i<j,
$$

are positive and the $n$ roots

$$
\alpha_{i}:=e_{i}-e_{i+1}, \quad i=1,2, \ldots, n-1, \quad \alpha_{n}=e_{n-1}+e_{n}
$$

constitute the system of simple roots.

If $\lambda=\sum_{i=1}^{n} m_{i} \boldsymbol{e}_{i}$ is a point of $E_{n}$, then it belongs to the dominant Weyl chamber $D_{+}$if and only if

$$
m_{1} \geq m_{2} \geq \cdots \geq m_{n-1} \geq\left|m_{n}\right| .
$$

If $\lambda=\sum_{i=1}^{n} \lambda_{i} \omega_{i}$, then the coordinates $\lambda_{i}$ in the $\omega$-basis are connected with the coordinates $m_{j}$ of $\lambda=\sum_{i=1}^{n} m_{i} \boldsymbol{e}_{i}$ by the formulas

$$
\begin{aligned}
& m_{1}=\lambda_{1}+\lambda_{2}+\cdots+\lambda_{n-2}+\frac{1}{2}\left(\lambda_{n-1}+\lambda_{n}\right) \text {, } \\
& m_{2}=\lambda_{2}+\cdots+\lambda_{n-2}+\frac{1}{2}\left(\lambda_{n-1}+\lambda_{n}\right) \text {, } \\
& \begin{array}{llllllll}
\ldots & \ldots & \ldots & \ldots & \ldots & \ldots & \ldots
\end{array} \\
& m_{n-1}=\quad \frac{1}{2}\left(\lambda_{n-1}+\lambda_{n}\right), \\
& m_{n}=\quad \frac{1}{2}\left(\lambda_{n-1}-\lambda_{n}\right),
\end{aligned}
$$


The inverse formulas are

$$
\lambda_{i}=m_{i}-m_{i+1}, \quad i=1,2, \ldots, n-2, \quad \lambda_{n-1}=m_{n-1}+m_{n}, \quad \lambda_{n}=m_{n-1}-m_{n} .
$$

By means of the formula (3.2) for the reflection $r_{\alpha}$ we find that $r_{\alpha}$ acts upon orthogonal coordinates of the vector $\lambda=\sum_{i=1}^{n} m_{i} \boldsymbol{e}_{i}$ by permuting the $i$-th and $j$-th coordinates if $\alpha=$ $\pm\left(\boldsymbol{e}_{i}-\boldsymbol{e}_{j}\right)$, as the permutation of $i$-th and $j$-th coordinates and the change of their signs if $\alpha= \pm\left(\boldsymbol{e}_{i}+\boldsymbol{e}_{j}\right)$. Thus, the Weyl group $W\left(D_{n}\right)$ consists of all permutations of the orthogonal coordinates $m_{1}, m_{2}, \ldots, m_{n}$ of $\lambda$ with sign alternations for an even number of coordinates.

The orbit $O(\lambda), \lambda=\left(m_{1}, m_{2}, \ldots, m_{n}\right), m_{1} \geq m_{2} \geq \cdots \geq m_{n-1} \geq\left|m_{n}\right|$, consists of all different points

$$
\left( \pm m_{i_{1}}, \pm m_{i_{2}}, \ldots, \pm m_{i_{n+1}}\right)
$$

obtained from $\left(m_{1}, m_{2}, \ldots, m_{n}\right)$ by permutations and alternations of even number of signs. The subgroup $W_{\lambda}$ of $W$ is a product of the Weyl group $W\left(D_{r}\right)$, where $r$ is the number of 0 's in the weight $\left(m_{1}, m_{2}, \ldots, m_{n}\right)$, and of the subgroups consisting of permutations of collections of coinciding non-vanishing $m$ 's. For example, the orbit $O(m, 0, \ldots, 0)$ consists of $2 n$ points $(0, \ldots, 0, \pm m, 0, \ldots, 0)$. The subgroup $W_{\lambda}$ is isomorphic to the Weyl group $W\left(D_{n-1}\right)$.

The orbit $O\left(m_{1}, m_{2}, 0, \ldots, 0\right), m_{1} \neq m_{2}$, consists of $4 n(n-1)$ points of the same form as in the case of $B_{n}$. The subgroup $W_{\lambda}$ is isomorphic to the Weyl group $W\left(D_{n-2}\right)$. If $m_{1}=m_{2}$, then this orbit consists of $2 n(n-1)$ elements.

If we have an orbit with points given in orthogonal coordinates, it is easy, by using formula (3.5), to transform it to $\omega$-coordinates.

\subsection{Orbits of $A_{3}$}

Orbits for $A_{3}, B_{3}$ and $C_{3}$ can be calculated by means of the description of Weyl groups $W\left(A_{3}\right)$, $W\left(B_{3}\right)$ and $W\left(C_{3}\right)$ in the orthogonal coordinate systems. Below we give results of such calculations. The points $\lambda$ of the orbits are given in the $\omega$-coordinates $(a b c)$, that is, $\lambda=a \omega_{1}+b \omega_{2}+c \omega_{3}$.

The orbit $O(a b c), a>0, b>0, c>0$, of $A_{3}$ contains the points

$$
\begin{aligned}
O(a b c) \ni & (a b c),(a+b-b b+c),(a+b c-b-c),(a b+c-c),(a+b+c-c-b), \\
& (a+b+c-b-c b),(-a a+b c),(-a a+b+c-c),(b-a-b a+b+c), \\
& (b+c-a-b-c a+b),(-a-b a b+c),(-b-a a+b+c)
\end{aligned}
$$

and the points, contragredient to these points, where the contragredient of the point $\left(a^{\prime} b^{\prime} c^{\prime}\right)$ is $\left(-c^{\prime}-b^{\prime}-a^{\prime}\right)$. We also have

$$
\begin{aligned}
& O\left(\begin{array}{lll}
a & b & 0
\end{array}\right) \ni\left(\begin{array}{lll}
a & b & 0
\end{array}\right),(a+b-b \quad b),(a+b 0-b),(-a a+b 0),(-a-b a b), \\
& (b-a-b a+b) \text { and contragredient points; } \\
& O\left(\begin{array}{lll}
a & 0 & c
\end{array}\right) \ni\left(\begin{array}{lll}
a & 0 & c
\end{array}\right),(a c-c),(a+c-c 0),(-a a c),(0-a a+c), \\
& (-a a+c-c) \text { and contragredient points; } \\
& O\left(\begin{array}{lll}
0 & b & c
\end{array}\right) \ni\left(\begin{array}{lll}
0 & b & c
\end{array}\right),(b-b b+c),(0 b+c-c),(b+c-b-c b),(-b 0 b+c), \\
& (b c-b-c) \text { and contragredient points; } \\
& O\left(\begin{array}{lll}
a & 0 & 0
\end{array}\right) \ni\left(\begin{array}{lll}
a & 0 & 0
\end{array}\right),\left(\begin{array}{lll}
-a & a & 0
\end{array}\right),\left(\begin{array}{lll}
0 & 0 & -a
\end{array}\right),\left(\begin{array}{lll}
0 & -a & a
\end{array}\right) \text {; } \\
& O\left(\begin{array}{lll}
0 & b & 0
\end{array}\right) \ni\left(\begin{array}{lll}
0 & b & 0
\end{array}\right),\left(\begin{array}{lll}
b & -b & b
\end{array}\right),\left(\begin{array}{lll}
b & 0 & -b
\end{array}\right),\left(\begin{array}{ll}
-b & b
\end{array}-b\right),\left(\begin{array}{lll}
0 & -b & 0
\end{array}\right),\left(\begin{array}{lll}
-b & 0 & b
\end{array}\right) \text {; } \\
& O\left(\begin{array}{lll}
0 & 0 & c
\end{array}\right) \ni\left(\begin{array}{lll}
0 & 0 & c
\end{array}\right),\left(\begin{array}{lll}
0 & c & -c
\end{array}\right),\left(\begin{array}{cll}
c & -c & 0
\end{array}\right),\left(\begin{array}{lll}
-c & 0 & 0
\end{array}\right) \text {. }
\end{aligned}
$$




\subsection{Orbits of $B_{3}$}

As in the previous case, the points $\lambda$ of the orbits are given by the coordinates $(a b c)$, where $\lambda=a \omega_{1}+b \omega_{2}+c \omega_{3}$. The orbit $O(a b c), a>0, b>0, c>0$, of $B_{3}$ contains the points

$$
\begin{aligned}
O(a b c) \ni & (a b c),(a+b-b 2 b+c),(-a a+b c),(b-a-b 2 a+2 b+c), \\
& (-a-b a 2 b+c),(-b-a 2 a+2 b+c),(a b+c-c),(a+b+c-b-c 2 b+c), \\
& (-a a+b+c-c),(b+c-a-b-c 2 a+2 b+c),(-a-b-c a 2 b+c), \\
& (-b-c-a 2 a+2 b+c),(-a-2 b-c b c),(-a-b-c-b 2 b+c), \\
& (a+2 b+c-a-b-c c),(b a+b+c-2 a-2 b-c),(a+b+c-a-2 b-c 2 b+c), \\
& (-b a+2 b+c-2 a-2 b-c),(-a-2 b-c b+c-c),(-a-b-b-c 2 b+c), \\
& (a+2 b+c-a-b-c),(b+c a+b-2 a-2 b-c),(a+b-a-2 b-c 2 b+c), \\
& (-b-c a+2 b+c-2 a-2 b-c)
\end{aligned}
$$

and also all these points with the minus sign. For the orbits $O(a b 0), O(a 0 c)$ and $O(0 b c)$ we have

$$
\begin{aligned}
O\left(\begin{array}{lll}
a & b & 0
\end{array}\right) \ni & \pm\left(\begin{array}{lll}
a & b & 0
\end{array}\right), \pm(a+b-b 2 b), \pm(-a a+b 0), \pm(b-a-b 2 a+2 b), \\
& \pm(-a-b a 2 b), \pm(-b-a 2 a+2 b), \pm(-a-2 b b 0), \pm(-a-b-b 2 b), \\
& \pm(a+2 b-a-b 0), \pm(b a+b-2 a-2 b), \pm(a+b-a-2 b 2 b), \pm(-b a+2 b-2 a-2 b) \\
O\left(\begin{array}{lll}
a & 0 & c
\end{array}\right) \ni & \pm\left(\begin{array}{lll}
a & 0 & c
\end{array}\right), \pm(-a a c), \pm(0-a 2 a+c), \pm(a c-c), \\
& \pm(a+c-c c), \pm(-a a+c-c), \pm(c-a-c 2 a+c), \pm(-a-c a c), \\
& \pm(-c-a 2 a+c), \pm(-a-c 0 c), \pm(a+c-a-c c), \pm(0 a+c-2 a-c) ; \\
O\left(\begin{array}{lll}
0 & b & c
\end{array}\right) \ni & \pm\left(\begin{array}{lll}
0 & b c
\end{array}\right), \pm(b-b 2 b+c), \pm(-b 02 b+c), \pm(0 b+c-c), \\
& \pm(b+c-b-c 2 b+c), \pm(-b-c 02 b+c), \pm(-2 b-c b c), \pm(-b-c-b 2 b+c), \\
& \pm(2 b+c-b-c c), \pm(b b+c-2 b-c), \pm(b+c-2 b-c 2 b+c), \pm(-b 2 b+c-2 b-c) .
\end{aligned}
$$

The orbits $O\left(\begin{array}{lll}a & 0 & 0\end{array}\right), O\left(\begin{array}{lll}0 & b & 0\end{array}\right)$ and $O\left(\begin{array}{lll}0 & 0 & c\end{array}\right)$ consist of the points

$$
\begin{aligned}
& O\left(\begin{array}{lll}
a & 0 & 0
\end{array}\right) \ni \pm\left(\begin{array}{lll}
a & 0 & 0
\end{array}\right), \pm\left(\begin{array}{lll}
a-a & 0
\end{array}\right), \pm\left(\begin{array}{lll}
0 & a & -2 a
\end{array}\right) \\
& O\left(\begin{array}{lll}
0 & b & 0
\end{array}\right) \ni \pm\left(\begin{array}{lll}
0 & b & 0
\end{array}\right), \pm\left(\begin{array}{lll}
b-b & 2 b
\end{array}\right), \pm\left(\begin{array}{lll}
-b & 0 & 2 b
\end{array}\right), \pm\left(\begin{array}{lll}
-2 b & b & 0
\end{array}\right), \pm\left(\begin{array}{lll}
-b-b & 2 b
\end{array}\right), \pm\left(\begin{array}{lll}
b-2 b & 2 b
\end{array}\right) \\
& O\left(\begin{array}{lll}
0 & 0 & c
\end{array}\right) \ni \pm\left(\begin{array}{lll}
0 & 0 & c
\end{array}\right), \pm\left(\begin{array}{lll}
c-c & c
\end{array}\right), \pm\left(\begin{array}{lll}
0 & c & -c
\end{array}\right), \pm\left(\begin{array}{lll}
-c & 0 & c
\end{array}\right) .
\end{aligned}
$$

\subsection{Orbits of $C_{3}$}

Again, the points $\lambda$ of the orbits are given by the coordinates $(a b c)$, where $\lambda=a \omega_{1}+b \omega_{2}+c \omega_{3}$. The orbit $O(a b c), a>0, b>0, c>0$, of $C_{3}$ contains the points

$$
\begin{aligned}
O(a b c) \ni & (a b c),(a+b-b b+c),(-a a+b c),(b-a-b a+b+c), \\
& (-a-b a b+c),(-b-a a+b+c),(a b+2 c-c),(a+b+2 c-b-2 c b+c), \\
& (-a a+b+2 c-c),(b+2 c-a-b-2 c a+b+c),(-a-b-2 c a b+c), \\
& (-b-2 c-a a+b+c),(-a-2 b-2 c b c),(-a-b-2 c-b b+c), \\
& (a+2 b+2 c-a-b-2 c c),(b a+b+2 c-a-b-c),(a+b+2 c-a-2 b-2 c b+c), \\
& (-b a+2 b+2 c-a-b-c),(-a-2 b-2 c b+2 c-c),(-a-b-b-2 c b+c), \\
& (a+2 b+2 c-a-b-c),(b+2 c a+b-a-b-c),(a+b-a-2 b-2 c b+c), \\
& (-b-2 c a+2 b+2 c-a-b-c)
\end{aligned}
$$


and also all these points with the minus sign. For the orbits $O(a b 0), O(a 0 c)$ and $O(0 b c)$ we have

$$
\begin{aligned}
O\left(\begin{array}{lll}
a & b & 0
\end{array}\right) \ni & \pm\left(\begin{array}{lll}
a & b & 0
\end{array}\right), \pm(a+b-b b), \pm(-a a+b 0), \pm(b-a-b a+b), \\
& \pm(-a-b a b), \pm(-b-a a+b), \pm(-a-2 b b 0), \pm(-a-b-b b), \\
& \pm(a+2 b-a-b 0), \pm(b a+b-a-b), \pm(a+b-a-2 b b), \pm(-b a+2 b-a-b) ; \\
O\left(\begin{array}{lll}
a & 0 & c
\end{array}\right) \ni & \pm\left(\begin{array}{lll}
a & 0 & c
\end{array}\right), \pm(-a a c), \pm(0-a a+c), \pm(a \quad 2 c-c), \\
& \pm(a+2 c-2 c c), \pm(a+2 c-a-2 c c), \pm(0 a+2 c-a-c), \pm(-a a+2 c-c), \\
& \pm(2 c-a-2 c a+c), \pm(-a-2 c a c), \pm(-2 c-a a+c), \pm(-a-2 c 0 c) ; \\
O\left(\begin{array}{lll}
0 & b & c
\end{array}\right) \ni & \pm(0 b c), \pm(b-b b+c), \pm(-b 0 b+c), \pm(0 b+2 c-c), \\
& \pm(b+2 c-b-2 c b+c), \pm(-b-2 c 0 b+c), \pm(-2 b-2 c b c), \pm(-b-2 c-b b+c), \\
& \pm(2 b+2 c-b-2 c c), \pm(b b+2 c-b-c), \pm(b+2 c-2 b-2 c b+c), \\
& \pm(-b 2 b+2 c-b-c) .
\end{aligned}
$$

The orbits $O\left(\begin{array}{lll}a & 0 & 0\end{array}\right), O\left(\begin{array}{lll}0 & b & 0\end{array}\right)$ and $O\left(\begin{array}{lll}0 & 0 & c\end{array}\right)$ consist of the points

$$
\begin{aligned}
& O\left(\begin{array}{lll}
a & 0 & 0
\end{array}\right) \ni \pm\left(\begin{array}{lll}
a & 0 & 0
\end{array}\right), \pm(a-a \quad 0), \pm\left(\begin{array}{lll}
0 & a & -a
\end{array}\right) \text {; }
\end{aligned}
$$

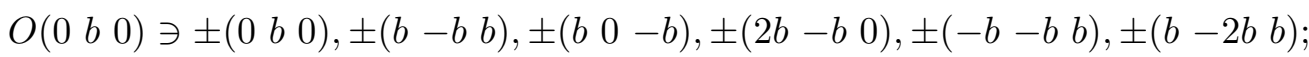

$$
\begin{aligned}
& O\left(\begin{array}{lll}
0 & 0 & c
\end{array}\right) \ni \pm\left(\begin{array}{lll}
0 & 0 & c
\end{array}\right), \pm\left(\begin{array}{lll}
0 & 2 c-c
\end{array}\right), \pm(2 c-2 c c), \pm(2 c 0-c) \text {. }
\end{aligned}
$$

\section{Operations on orbits}

In this section we consider some operations with orbits: decomposition of products of orbits into orbits and decomposition of $W$-orbits into $W^{\prime}$-orbits. These operations are a base for corresponding operations with orbit functions.

\subsection{Decomposition of products of orbits}

The product $O(\lambda) \otimes O\left(\lambda^{\prime}\right)$ of two orbits $O(\lambda)$ and $O\left(\lambda^{\prime}\right)$ is the set of all points of the form $\lambda_{1}+\lambda_{2}$, where $\lambda_{1} \in O(\lambda)$ and $\lambda_{2} \in O\left(\lambda^{\prime}\right)$. Since a set of points $\lambda_{1}+\lambda_{2}, \lambda_{1} \in O(\lambda), \lambda_{2} \in O\left(\lambda^{\prime}\right)$, is invariant with respect to action of the corresponding Weyl group, each product of orbits is decomposable into a sum of orbits. If $\lambda=0$, then $O(\lambda) \otimes O\left(\lambda^{\prime}\right)=O\left(\lambda^{\prime}\right)$. If $\lambda^{\prime}=0$, then $O(\lambda) \otimes O\left(\lambda^{\prime}\right)=O(\lambda)$. In what follows we assume that $\lambda \neq 0$ and $\lambda^{\prime} \neq 0$. Let us consider decompositions of products of orbits.

Example. Orbits of $A_{1}$. If $a \in E_{1}$, then the orbit of this point $O(a)$ consists of two points $a$ and $-a$. It is easy to see that for the product $O(a) \otimes O(b), a \neq b$, we have

$$
\begin{aligned}
O(a) \otimes O(b) & \equiv\{a,-a\} \otimes\{b,-b\}=\{a+b,-a-b\} \cup\{|a-b|,-|a-b|\} \\
& =O(a+b) \cup O(|a-b|) .
\end{aligned}
$$

However, decomposition of products of orbits in higher dimension of Euclidean spaces is not a simple task. We first consider some general results on decomposition.

Let $O(\lambda)=\left\{w \lambda \mid w \in W / W_{\lambda}\right\}$ and $O(\mu)=\left\{w \mu \mid w \in W / W_{\mu}\right\}$ be two orbits. Then

$$
\begin{aligned}
O(\lambda) & \otimes O(\mu)=\left\{w \lambda+w^{\prime} \mu \mid w \in W / W_{\lambda}, w^{\prime} \in W / W_{\mu}\right\} \\
& =\left\{w \lambda+w_{1} \mu \mid w \in W / W_{\lambda}\right\} \cup\left\{w \lambda+w_{2} \mu \mid w \in W / W_{\lambda}\right\} \cup \cdots \cup\left\{w \lambda+w_{s} \mu \mid w \in W / W_{\lambda}\right\},
\end{aligned}
$$

where $w_{1}, w_{2}, \ldots, w_{s}$ is the set of elements of $W / W_{\mu}$. Since a product of orbits is invariant with respect to $W$, for decomposition of the product $O(\lambda) \otimes O(\mu)$ into separate orbits it is necessary 
to take dominant elements from each term of the right hand side of $(4.1)$. That is, $O(\lambda) \otimes O(\mu)$ is a union of the orbits, determined by points from

$$
\begin{gathered}
D\left(\left\{w \lambda+w_{1} \mu \mid w \in W / W_{\lambda}\right\}\right), \quad D\left(\left\{w \lambda+w_{2} \mu \mid w \in W / W_{\lambda}\right\}\right), \quad \ldots, \\
D\left(\left\{w \lambda+w_{s} \mu \mid w \in W / W_{\lambda}\right\}\right),
\end{gathered}
$$

where $D\left(\left\{w \lambda+w_{i} \mu \mid w \in W / W_{\lambda}\right\}\right)$ means the set of dominant elements in the collection $\{w \lambda+$ $\left.w_{i} \mu \mid w \in W / W_{\lambda}\right\}$.

Proposition 1. The product $O(\lambda) \otimes O(\mu)$ consists only of orbits of the form $O(|w \lambda+\mu|)$, $w \in W / W_{\lambda}$, where $|w \lambda+\mu|$ is a dominant weight of the orbit containing $w \lambda+\mu$. Moreover, each such orbit $O(|w \lambda+\mu|), w \in W / W_{\lambda}$, belongs to the product $O(\lambda) \otimes O(\mu)$.

Proof. For each dominant element $w \lambda+w_{i} \mu$ from (4.2) there exists an element $w^{\prime \prime} \in W$ such that $w^{\prime \prime}\left(w \lambda+w_{i} \mu\right)=w^{\prime} \lambda+\mu$. It means that $w \lambda+w_{i} \mu$ is of the form $\left|w^{\prime} \lambda+\mu\right|, w^{\prime} \in W / W_{\lambda}$. Conversely, take any element $w \lambda+\mu, w \in W / W_{\lambda}$. It belongs to the product $O(\lambda) \otimes O(\mu)$. This means that $|w \lambda+\mu|$ also belongs to this product. Therefore, the orbit $O(|w \lambda+\mu|)$ is contained in $O(\lambda) \otimes O(\mu)$. The proposition is proved.

It follows from Proposition 1 that for decomposition of the product $O(\lambda) \otimes O(\mu)$ into separate orbits we have to take all elements $w \lambda+\mu, w \in W / W_{\lambda}$, and to find the corresponding dominant elements $|w \lambda+\mu|, w \in W / W_{\lambda}$. It may seem that

$$
O(\lambda) \otimes O(\mu)=\bigcup_{w \in W / W_{\lambda}} O(|w \lambda+\mu|) .
$$

However, this is not true. The simplest counterexample is when $\mu=0$. Then according to this formula $O(\lambda) \otimes O(\mu)=O(\lambda) \cup O(\lambda) \cup \cdots \cup O(\lambda)$ ( $\left|W / W_{\lambda}\right|$ times). However, as we know, $O(\lambda) \otimes O(\mu)=O(\lambda)$.

Proposition 1 states that instead of (4.3) we have

$$
O(\lambda) \otimes O(\mu) \subseteq \bigcup_{w \in W / W_{\lambda}} O(|w \lambda+\mu|) .
$$

Note that some orbits on the right hand side can coincide. Some orbits can be contained in the decomposition of $O(\lambda) \otimes O(\mu)$ with a multiplicity. The most difficult problem under consideration of products of orbits is to find these multiplicities.

Proposition 2. Let $O(\lambda)$ and $O(\mu)$ be orbits such that $\lambda \neq 0$ and $\mu \neq 0$, and let all elements $w \lambda+\mu, w \in W / W_{\lambda}$, be strictly dominant (that is, they are dominant and do not belong to any wall of Weyl dominant chamber). Then

$$
O(\lambda) \otimes O(\mu)=\bigcup_{w \in W / W_{\lambda}} O(w \lambda+\mu) .
$$

Proof. Under the conditions of the proposition the set of elements $w \lambda+\mu, w \in W / W_{\lambda}$, are contained in the first set of (4.2) if $w_{1}$ coincides with the identical transformation. Moreover, $W_{w \lambda+\mu}=\{1\}$ for all elements $w \in W / W_{\lambda}$. Then $\mu$ is a strictly dominant element, that is, $W_{\mu}=\{1\}$. Let us show that the collection (4.2) contains only one non-empty set $D(\{w \lambda+\mu \mid w \in$ $\left.\left.W / W_{\lambda}\right\}\right)$. Indeed, let $w \lambda+w_{i} \mu, w_{i} \neq 1$, be a dominant element. Then

$$
w_{i}^{-1}\left(w \lambda+w_{i} \mu\right)=w_{i}^{-1} w \lambda+\mu
$$

is not a dominant element since $W_{w_{i}^{-1} w \lambda+\mu}=\{1\}$. This contradicts the conditions of the proposition and, therefore, the collection (4.2) contains only one non-empty set. The set of orbits, corresponding to the points of $D\left(\left\{w \lambda+\mu \mid w \in W / W_{\lambda}\right\}\right)$, coincides with the right hand side of (4.5). The proposition is proved. 
Under the conditions of Proposition 2, elements $w \lambda+w^{\prime} \mu, w \in W / W_{\lambda}, w^{\prime} \in W$, are pairwise different, and all dominant elements of $O(\lambda) \otimes O(\mu)$ are contained in one set of (4.2). In general, some elements $|w \lambda+\mu|$ with fixed $w$ can be contained in several sets of (4.2), that is, some orbits from Proposition 1 can be contained in $O(\lambda) \otimes O(\mu)$ with a multiplicity $m_{|w \lambda+\mu|}>1$. Then there exist dominant elements $w^{\prime} \lambda+w_{i} \mu$ and $w^{\prime \prime} \lambda+w_{j} \mu$ from different sets of (4.2) such that

$$
w^{\prime} \lambda+w_{i} \mu=w^{\prime \prime} \lambda+w_{j} \mu=|w \lambda+\mu|
$$

for some $w \in W / W_{\lambda}$ (one of these elements can coincide with $w \lambda+\mu$ if $w \lambda+\mu$ is dominant).

Proposition 3. Let $O(\lambda)$ and $O(\mu)$ be orbits such that $\lambda \neq 0$ and $\mu \neq 0$, and let all elements $w \lambda+\mu, w \in W / W_{\lambda}$, be dominant. Then

$$
O(\lambda) \otimes O(\mu)=\bigcup_{w \in W / W_{\lambda}} n_{w \lambda+\mu} O(w \lambda+\mu)
$$

where $n_{w \lambda+\mu}=\left|W_{w \lambda+\mu}\right|$.

Proof. Since $\lambda \neq 0$, all elements $w \lambda+\mu, w \in W / W_{\lambda}$, can be dominant if and only if $W_{\mu}=\{1\}$. Then on the right hand side of (4.1) there are $|W|$ terms. If the element $w \lambda+\mu$ is strictly dominant, that is, $W_{w \lambda+\mu}=\{1\}$, then this element is met only in one term. This means that $n_{w \lambda+\mu}=1$. If $w \lambda+\mu$ is placed on some Weyl wall, then it is met in $n_{w \lambda+\mu}=\left|W_{w \lambda+\mu}\right|$ terms. Therefore, there are $n_{w \lambda+\mu}$ orbits $O(w \lambda+\mu)$ in the decomposition of $O(\lambda) \otimes O(\mu)$. The proposition is proved.

The highest and the lowest components of the decomposition of $O(\lambda) \otimes O(\mu)$ can be easily found. The highest one is $O(\lambda+\mu)$. The lowest one is $O(|\lambda+\widetilde{\mu}|)=O(|\widetilde{\lambda}+\mu|)$, where the tilde denotes the lowest weight of the orbit and, as before, $|\lambda+\widetilde{\mu}|$ denotes a dominant element of the orbit containing $\lambda+\widetilde{\mu}$ (it often happens that $\lambda+\widetilde{\mu}$ is not dominant). The highest component $O(\lambda+\mu)$ is always with multiplicity 1 in the decomposition. The lowest one may not be unique.

By Proposition 1, all other orbits in the decomposition of $O(\lambda) \otimes O(\mu)$ are placed between the orbits $O(\lambda+\mu)$ and $O(|\lambda+\widetilde{\mu}|)$. This means that dominant elements $|w \lambda+\mu|$ of other orbits $O(|w \lambda+\mu|), w \in W / W_{\lambda}$, are in the intersection of the sets $\left\{\lambda+\mu-\sum_{i} m_{i} \alpha_{i} \mid m_{i} \in \mathbb{N}\right\}$ and $\left\{|\widetilde{\lambda}+\mu|+\sum_{i} m_{i} \alpha_{i} \mid m_{i} \in \mathbb{N}\right\}$, where $\left\{\alpha_{1}, \alpha_{2}, \ldots, \alpha_{n}\right\}$ is a set of simple roots.

Proposition 4. If $W_{\mu}=\{1\}$ and none of the points $w \lambda+\mu, w \in W$, lie on some Weyl chamber, then

$$
O(\lambda) \otimes O(\mu)=\bigcup_{w \in W / W_{\lambda}} O(|w \lambda+\mu|) .
$$

Proof. For the product $O(\lambda) \times O(\mu)$ the inclusion (4.4) takes place. Each orbit $O(|w \lambda+\mu|)$, $w \in W$, has $|W|$ elements and is contained in $O(\lambda) \times O(\mu)$. Therefore, numbers of elements in both sides of (4.4) coincide. This means that the inclusion (4.4) is in fact an equality. This proves the proposition.

We formulate 2 conjectures concerning decomposition of products of orbits.

Conjecture 1. Let $O(\lambda)$ and $O(\mu), \lambda \neq 0, \mu \neq 0$, be orbits, and let $\mu$ be strictly dominant. If for some $w \in W$ the element $w \lambda+\mu$ is strictly dominant, then the multiplicity of $O(w \lambda+\mu)$ in $O(\lambda) \otimes O(\mu)$ is 1 . 
Conjecture 2. Let $O(\lambda)$ and $O(\mu), \lambda \neq 0, \mu \neq 0$, be orbits, and let a stabilizer subgroup of $\mu$ be generated by the reflections $r_{i_{1}}, \ldots, r_{i_{s}}$, corresponding to simple roots $\alpha_{i_{1}}, \ldots, \alpha_{i_{s}}$. If for each $w \in W / W_{\lambda}$ we have $\left\langle w \lambda+\mu, \alpha_{j}\right\rangle>0, j \notin\left\{i_{1}, i_{2}, \ldots, i_{s}\right\}$, then

$$
O(\lambda) \otimes O(\mu)=\bigcup_{w \in W / W_{\lambda}} O(w \lambda+\mu)
$$

At the end of this subsection we formulate the following method for decomposition of products $O(\lambda) \otimes O(\mu)$, which follows from the statement of Proposition 1. On the first step we shift all points of the orbit $O(\lambda)$ by $\mu$. As a result, we obtain the set of points $w \lambda+\mu, w \in$ $W / W_{\lambda}$. On the second step, we map non-dominant elements of this set by elements of the Weyl group $W$ to the dominant chamber. On this step we obtain the set $|w \lambda+\mu|, w \in W / W_{\lambda}$. Then according to Proposition $1, O(\lambda) \otimes O(\mu)$ consists of the orbits $O(|w \lambda+\mu|)$. On the third step, we determine multiplicities of these orbits, taking into account the above propositions or making direct calculations.

\subsection{Decomposition of products for rank 2}

Here we give examples of decompositions of products of orbits for the cases $A_{2}, B_{2}$ and $G_{2}$. Orbits for these cases are placed on the plane. Therefore, decompositions can be done by geometrical calculations on this plane. The cases of $A_{2}$ and $B_{2}$ can be easily considered by using for points of orbits orthogonal coordinates from Section 3. For these coordinates the corresponding Weyl groups have a simple description and this give the possibility to make calculations in a simple manner.

For the case of $A_{2}$ we have

$$
\begin{aligned}
& A_{2}: O(a 0) \otimes O(b 0)=O(a+b 0) \cup O(-a+b a) \quad(a<b) \\
& O(a 0) \otimes O(a 0)=O(2 a 0) \cup 2 O(0 a) \\
& O(a 0) \otimes O(0 b)=O(a b) \cup O(0-a+b) \quad(a<b) \\
& O(a 0) \otimes O(0 a)=O(a \quad a) \cup 3 O\left(\begin{array}{ll}
0 & 0
\end{array}\right) \\
& O(a 0) \otimes O(b b)=O(a+b b) \cup O(a-2 b b) \cup O(a-b 2 b) \quad(a>2 b) \\
& O(a 0) \otimes O(b b)=O(a+b b) \cup O(2 b-a a-b) \cup O(a-b 2 b) \quad(2 b>a>b) \\
& O(a 0) \otimes O(b b)=O(a+b b) \cup O(b-a a+b) \cup O(b b-a) \quad(b>a) \\
& O\left(\begin{array}{ll}
1 & 1
\end{array}\right) \otimes O\left(\begin{array}{ll}
1 & 1
\end{array}\right)=O(2 \quad 2) \cup 2 O\left(\begin{array}{ll}
0 & 3
\end{array}\right) \cup 2 O\left(\begin{array}{lll}
3 & 0) \cup 2 O(1 & 1
\end{array}\right) \\
& \cup 6 O(00) \text {. }
\end{aligned}
$$

Similar products of $C_{2}$ orbits are of the form

$$
\begin{aligned}
& C_{2}: O(a 0) \otimes O(b 0)=O(a+b 0) \cup O(a-b 0) \cup O(a-b b) \quad(a>b) \\
& O(a 0) \otimes O(a 0)=O(2 a 0) \cup 2 O\left(\begin{array}{ll}
0 & 2 a) \cup 4 O(00)
\end{array}\right. \\
& O(0 a) \otimes O(0 b)=O(0 a+b) \cup O(2 b a-b) \cup O(0 a-b) \quad(a>b) \\
& O\left(\begin{array}{ll}
0 & a
\end{array}\right) \otimes O\left(\begin{array}{ll}
0 & a
\end{array}\right)=O\left(\begin{array}{ll}
0 & 2 a
\end{array}\right) \cup 2 O(2 a \quad 0) \cup 4 O\left(\begin{array}{ll}
0 & 0
\end{array}\right) \\
& O(a 0) \otimes O(0 b)=O(a b) \cup O(a-2 b b) \quad(a>2 b) \\
& O(a 0) \otimes O(0 b)=O(a b) \cup O(2 b-a a-b) \quad(2 b>a>b) \\
& O(a 0) \otimes O(0 b)=O(a b) \cup O(a b-a) \quad(b>a) \\
& O(a 0) \otimes O(0 a)=O(a a) \cup 2 O(a 0) \\
& O(a 0) \otimes O(02 a)=O(a 2 a) \cup O(a a) \\
& O(2 a 0) \otimes O(0 a)=O(2 a a) \cup 2 O(0 a) \text {. }
\end{aligned}
$$


Finally a few products of $G_{2}$ orbits:

$$
\begin{aligned}
& G_{2}: O(a 0) \otimes O(b 0)=O(a+b 0) \cup O(b-a 3 a) \cup O(2 a-b 3 b-3 a) \\
& \cup O(b-a 0) \quad(a<b<2 a) \\
& O(a \quad 0) \otimes O(b 0)=O(a+b 0) \cup O(b-a 3 a) \cup O(b-2 a 3 a) \\
& \cup O(b-a 0) \quad(b>2 a) \\
& O(a 0) \otimes O(a 0)=O(2 a 0) \cup 2 O\left(\begin{array}{lll}
0 & 3 a) \cup 2 O(a 0) \cup 6 O(00)
\end{array}\right. \\
& O(a 0) \otimes O(2 a 0)=O(3 a 0) \cup O(a 0) \cup O(a 3 a) \cup 2 O\left(\begin{array}{ll}
0 & 3 a
\end{array}\right) \\
& O(0 a) \otimes O(0 b)=O(0 a+b) \cup O(a b-a) \cup O(b-a 2 a-b) \\
& \cup O(0 b-a) \quad(a<b<2 a) \\
& O(0 a) \otimes O(0 b)=O(0 a+b) \cup O(0 b-a) \cup O(a b-a) \\
& \cup O(a b-2 a) \quad(b>2 a)
\end{aligned}
$$

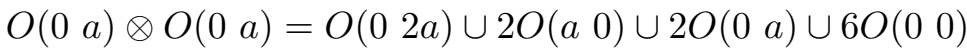

$$
\begin{aligned}
& O(0 a) \otimes O(02 a)=O\left(\begin{array}{ll}
0 & 3 a
\end{array}\right) \cup 2 O(a \quad 0) \cup O(a a) \cup O\left(\begin{array}{ll}
0 & a
\end{array}\right) .
\end{aligned}
$$

Let us note that the most complicated case for decomposition of products of orbits is for $E_{8}$. Products of all 36 pairs of orbits of fundamental weights of $E_{8}$ are fully decomposed in [14], as a sideline to the decomposition of products of fundamental representations of $E_{8}$.

\subsection{Decomposition of $W$-orbits into $W^{\prime}$-orbits}

Let $R$ be a root system with the Weyl group $W$. Let $R^{\prime}$ be another root system such that $R^{\prime}$ is a subset of $R$. Then the Weyl group $W^{\prime}$ of $R^{\prime}$ can be considered as a subgroup of $W$.

Let $O_{W}(\lambda)$ be a $W$-orbit. The set of elements of $O_{W}(\lambda)$ is invariant with respect to $W^{\prime}$, that is, $w^{\prime} O_{W}(\lambda)=O_{W}(\lambda), w^{\prime} \in W^{\prime}$. This means that $O_{W}(\lambda)$ consists of $W^{\prime}$-orbits. It is an important problem to represent $O_{W}(\lambda)$ as a union of $W^{\prime}$-orbits. Properties of such representations depend on the root systems $R$ and $R^{\prime}$ (or on the Weyl groups $W$ and $W^{\prime}$ ). We distinguish two cases:

Case 1: The root systems $R$ and $R^{\prime}$ span vector spaces of the same dimension. In this case the Weyl chambers for $W$ are smaller than the Weyl chambers for $W^{\prime}$. Moreover, each Weyl chamber of $W^{\prime}$ consists of $\left|W / W^{\prime}\right|$ chambers of $W$. Let $D_{+}$be a dominant Weyl chamber of the root system $R$. Then a dominant Weyl chamber of $W^{\prime}$ consists of $W$-chambers $w_{i} D_{+}$, $i=1,2, \ldots, k, k=\left|W / W^{\prime}\right|$, where $w_{i}, i=1,2, \ldots, k$, are representatives of all cosets in $W / W^{\prime}$. If $\lambda$ do not lie on any wall of the dominant Weyl chamber $D_{+}$, then

$$
O_{W}(\lambda)=\bigcup_{i=1}^{k} O_{W^{\prime}}\left(w_{i} \lambda\right),
$$

where $O_{W^{\prime}}$ are $W^{\prime}$-orbits. When representing $\lambda$ by coordinates in the $\omega$-basis it is necessary to take into account that the coordinates of the same point in $\omega$-bases related to the root systems $R$ and $R^{\prime}$ are different. There exist matrices mapping coordinates in these different $\omega$-bases (see Subsection 4.10 below).

Case 2: The root systems $R$ and $R^{\prime}$ span vector spaces of different dimensions. This case is more complicated. In order to represent $O_{W}(\lambda)$ as a union of $W^{\prime}$-orbits, it is necessary to take the projection of the points $\mu$ of $O_{W}(\lambda)$ to the vector subspace $E_{n^{\prime}}$ spanned by $R^{\prime}$ and to select in that set of projected points dominant points with respect to the root system $R^{\prime}$. Note that, under projection, different points of $O_{W}(\lambda)$ can give the same point in $E_{n^{\prime}}$. This leads to coinciding $W^{\prime}$-orbits in the representation of $O_{W}(\lambda)$ as a union of $W^{\prime}$-orbits. 


\subsection{Decomposition of $W\left(A_{n}\right)$-orbits into $W\left(A_{n-1}\right)$-orbits}

For such decomposition it is convenient to represent orbit elements in an orthogonal coordinate system (see Subsection 3.1). Let $O(\lambda) \equiv O\left(m_{1}, \ldots, m_{n+1}\right)$ be a $W\left(A_{n}\right)$-orbit with dominant element $\lambda=\left(m_{1}, m_{2}, \ldots, m_{n+1}\right)$. The orthogonal coordinates $m_{1}, m_{2}, \ldots, m_{n+1}$ satisfy the condition $m_{1}+m_{2}+\cdots+m_{n+1}=0$. However, as we noted in Subsection 3.1, we may add to all coordinates $m_{i}$ the same real number, since under this the $\omega$-coordinates $\lambda_{i}=m_{i}-m_{i+1}$, $i=1,2, \ldots, n$ do not change. This note is important under decomposition of $W\left(A_{n}\right)$-orbits into $W\left(A_{n-1}\right)$-orbits since, under this the condition $x_{1}+x_{2}+\cdots+x_{n}=0$ can be violated. This does not change the result for $\omega$-coordinates.

Let us suppose that the dominant element $\lambda=\left(m_{1}, m_{2}, \ldots, m_{n+1}\right)$ does not lie on a wall of the dominant Weyl chamber, that is,

$$
m_{1}>m_{2}>\cdots>m_{n}>m_{n+1} .
$$

The orbit $O(\lambda)$ consists of all points

$$
w\left(m_{1}, m_{2}, \ldots, m_{n+1}\right)=\left(m_{i_{1}}, m_{i_{2}}, \ldots, m_{i_{n+1}}\right), \quad w \in W\left(A_{n}\right),
$$

where $\left(i_{1}, i_{2}, \ldots, i_{n+1}\right)$ is a permutation of the numbers $1,2, \ldots, n+1$. Points of $O(\lambda)$ belong to the vector space $E_{n+1}$. We restrict these points to the vector subspace $E_{n}$, spanned by the simple roots $\alpha_{1}, \alpha_{2}, \ldots, \alpha_{n-1}$ of $A_{n}$, which form a set of simple roots of $A_{n-1}$. This restriction then reduces to removing the last coordinate $m_{i_{n+1}}$ in points $\left(m_{i_{1}}, m_{i_{2}}, \ldots, m_{i_{n+1}}\right)$ of the orbit $O(\lambda)$ (see (4.6)). As a result, we obtain the set of points

$$
\left(m_{i_{1}}, m_{i_{2}}, \ldots, m_{i_{n}}\right)
$$

from the points (4.6). The point (4.7) is dominant if and only if

$$
m_{i_{1}} \geq m_{i_{2}} \geq \cdots \geq m_{i_{n}}
$$

(in fact, for the points (4.6) equalities here are excluded). It is easy to see that (after restriction to $E_{n}$, that is, if we remove the last coordinate) the set (4.6) contains the following set of dominant elements:

$$
\left(m_{1}, \ldots, m_{i-1}, \hat{m}_{i}, m_{i+1}, \ldots, m_{n+1}\right), \quad i=1,2, \ldots, n+1,
$$

where a hat over $m_{i}$ means that the coordinate $m_{i}$ must be omitted. Thus, the $W\left(A_{n}\right)$-orbit $O\left(m_{1}, m_{2}, \ldots, m_{n+1}\right)$ with $m_{1}>m_{2}>\cdots>m_{n+1}$ consists of the following $W\left(A_{n-1}\right)$-orbits:

$$
O\left(m_{1}, \ldots, m_{i-1}, \hat{m}_{i}, m_{i+1}, \ldots, m_{n+1}\right), \quad i=1,2, \ldots, n+1 .
$$

All these orbits are contained in $O\left(m_{1}, m_{2}, \ldots, m_{n+1}\right)$ with multiplicity 1 . By using formula (3.1) it is easy to represent the dominant elements of (4.8) in $\omega$-coordinates.

\subsection{Decomposition of $W\left(A_{n-1}\right)$-orbits into $W\left(A_{p-1}\right) \times W\left(A_{q-1}\right)$-orbits, $p+q=n$}

Again we use orthogonal coordinates to represent orbit elements. We take in the system of simple roots $\alpha_{1}, \alpha_{2}, \ldots, \alpha_{n-1}$ of $A_{n-1}$ two parts: $\alpha_{1}, \alpha_{2}, \ldots, \alpha_{p-1}$ and $\alpha_{p+1}, \alpha_{p+2}, \ldots, \alpha_{p+q-1} \equiv \alpha_{n-1}$. The first part determines $W\left(A_{p-1}\right)$ and the second part generates $W\left(A_{q-1}\right)$. Again we suppose that the orthogonal coordinates $m_{1}, m_{2}, \ldots, m_{n}$ of $\lambda$, determining the $W\left(A_{n-1}\right)$-orbit $O(\lambda)$, satisfy the condition

$$
m_{1}>m_{2}>\cdots>m_{n}
$$


that is, $\lambda$ does not lie on a wall of the dominant chamber. The orbit $O(\lambda)$ consists of all points

$$
w\left(m_{1}, m_{2}, \ldots, m_{n}\right)=\left(m_{i_{1}}, m_{i_{2}}, \ldots, m_{i_{n}}\right), \quad w \in W\left(A_{n-1}\right),
$$

where $\left(i_{1}, i_{2}, \ldots, i_{n}\right)$ is a permutation of the numbers $1,2, \ldots, n$. We restrict points (4.9) to the vector subspace $E_{p} \times E_{q}$ spanned by the simple roots $\alpha_{1}, \alpha_{2}, \ldots, \alpha_{p-1}$ and $\alpha_{p+1}, \alpha_{p+2}, \ldots, \alpha_{n-1}$, respectively. Under restriction to $E_{p} \times E_{q}$ the point (4.9) becomes the point

$$
\left(m_{i_{1}}, m_{i_{2}}, \ldots, m_{i_{p}}\right)\left(m_{i_{p+1}}, m_{i_{p+2}}, \ldots, m_{i_{n}}\right) .
$$

In order to determine the set of $W\left(A_{p-1}\right) \times W\left(A_{q-1}\right)$-orbits contained in $O(\lambda)$ we have to choose in (4.9) all elements for which

$$
m_{i_{1}}>m_{i_{2}}>\cdots>m_{i_{p}}, \quad m_{i_{p+1}}>m_{i_{p+2}}>\cdots>m_{i_{n}}
$$

To find this set we have to take all possible sets of numbers $m_{i_{1}}, m_{i_{2}}, \ldots, m_{i_{p}}$ in the set $m_{1}, m_{2}, \ldots, m_{n}$, such that $m_{i_{1}}>m_{i_{2}}>\cdots>m_{i_{p}}$. Let $\Sigma$ denotes the collection of such sets. Then $O(\lambda)$ consists of $W\left(A_{p-1}\right) \times W\left(A_{q-1}\right)$-orbits

$$
O\left(\left(m_{i_{1}}, m_{i_{2}}, \ldots, m_{i_{p}}\right)\left(m_{j_{1}}, m_{j_{2}}, \ldots, m_{j_{q}}\right)\right), \quad\left(m_{i_{1}}, m_{i_{2}}, \ldots, m_{i_{p}}\right) \in \Sigma,
$$

where $\left(m_{j_{1}}, m_{j_{2}}, \ldots, m_{j_{q}}\right)$ is a supplement of $\left(m_{i_{1}}, m_{i_{2}}, \ldots, m_{i_{p}}\right)$ in the set $\left(m_{1}, m_{2}, \ldots, m_{n}\right)$, taken in such an order that $m_{j_{1}}>m_{j_{2}}>\cdots>m_{j_{q}}$. Each of these $W\left(A_{p-1}\right) \times W\left(A_{q-1}\right)$-orbits is contained in $O(\lambda)$ only once.

\subsection{Decomposition of $W\left(B_{n}\right)$-orbits into $W\left(B_{n-1}\right)$-orbits and of $W\left(C_{n}\right)$-orbits into $W\left(C_{n-1}\right)$-orbits}

Decomposition of $W\left(B_{n}\right)$-orbits and decomposition of $W\left(C_{n}\right)$-orbits are achieved in the same way. For this reason, we give a proof only in the case of $W\left(C_{n}\right)$-orbits.

We know that $C_{n}$ is defined by simple roots $\alpha_{1}, \alpha_{2}, \ldots, \alpha_{n}$. The roots $\alpha_{2}, \ldots, \alpha_{n}$ is the set of simple roots of $C_{n-1}$. They span the subspace $E_{n-1}$.

In order to determine elements $\lambda$ of $E_{n}$ we use the orthogonal coordinates $m_{1}, m_{2}, \ldots, m_{n}$ (see Subsection 3.3). Then $\lambda$ is dominant if $m_{1} \geq m_{2} \geq \cdots \geq m_{n} \geq 0$. We assume that $\lambda$ satisfies the condition

$$
m_{1}>m_{2}>\cdots>m_{n}>0 \text {. }
$$

Then the orbit $O(\lambda)$ consists of all points

$$
w\left(m_{1}, m_{2}, \ldots, m_{n}\right)=\left( \pm m_{i_{1}}, \pm m_{i_{2}}, \ldots, \pm m_{i_{n}}\right), \quad w \in W\left(C_{n}\right),
$$

where $\left(i_{1}, i_{2}, \ldots, i_{n}\right)$ is a permutation of the numbers $1,2, \ldots, n$ (all combinations of signs are possible).

Restricting the elements (4.10) to the vector subspace $E_{n-1}$, defined above, is reduced to removing the first coordinate $\pm m_{i_{1}}$ in (4.10). As a result, we obtain from the set (4.10) the collection

$$
\left( \pm m_{i_{2}}, \pm m_{i_{3}}, \ldots, \pm m_{i_{n}}\right), \quad w \in W\left(C_{n}\right) .
$$

Only the points $\left(m_{i_{2}}, m_{i_{3}}, \ldots, m_{i_{n-1}}, \pm m_{i_{n}}\right)$ may be dominant. Moreover, such a point is dominant if and only if

$$
m_{i_{2}}>m_{i_{3}}>\cdots>m_{i_{n}}
$$


Therefore, under restriction of points (4.10) to $E_{n-1}$, we obtain the following $W\left(C_{n-1}\right)$-dominant elements:

$$
\left(m_{1}, m_{2}, \ldots, m_{i-1}, \hat{m}_{i}, m_{i+1}, \ldots, m_{n}\right), \quad i=1,2, \ldots, n
$$

where a hat over $m_{i}$ means that the coordinate $m_{i}$ must be omitted. Moreover, the element (4.11) with fixed $i$ can be obtained from two elements in (4.10), namely, from $\left(m_{1}, m_{2}, \ldots, m_{i-1}, \pm m_{i}\right.$, $\left.m_{i+1}, \ldots, m_{n}\right)$.

Thus, the $W\left(C_{n}\right)$-orbits $O\left(m_{1}, m_{2}, \ldots, m_{n}\right)$ with $m_{1}>m_{2}>\cdots>m_{n}>0$ consists of the following $W\left(C_{n-1}\right)$-orbits:

$$
O\left(m_{1}, m_{2}, \ldots, m_{i-1}, \hat{m}_{i}, m_{i+1}, \ldots, m_{n}\right), \quad i=1,2, \ldots, n .
$$

Each such $W\left(C_{n-1}\right)$-orbit is contained in $O\left(m_{1}, m_{2}, \ldots, m_{n}\right)$ with multiplicity 2.

For $W\left(B_{n}\right)$-orbits we have a similar assertion: $A W\left(B_{n}\right)$-orbits $O\left(m_{1}, m_{2}, \ldots, m_{n}\right)$ with $m_{1}>$ $m_{2}>\cdots>m_{n}>0$ consists of $W\left(B_{n-1}\right)$-orbits

$$
O\left(m_{1}, m_{2}, \ldots, m_{i-1}, \hat{m}_{i}, m_{i+1}, \ldots, m_{n}\right), \quad i=1,2, \ldots, n,
$$

and each such orbit is contained in the decomposition with multiplicity 2.

\subsection{Decomposition of $W\left(C_{n}\right)$-orbits into $W\left(A_{p-1}\right) \times W\left(C_{q}\right)$-orbits, $p+q=n$}

If $\alpha_{1}, \alpha_{2}, \ldots, \alpha_{n}$ are simple roots for $C_{n}$, then $\alpha_{1}, \alpha_{2}, \ldots, \alpha_{p-1}$ are simple roots for $A_{p-1}$ (they can be embedded into the linear subspace $\left.E_{p}\right)$ and $\alpha_{p+1}, \alpha_{p+2}, \ldots, \alpha_{n}$ are simple roots for $C_{q}$ (they generate the linear subspace $E_{q}$ ).

We use orthogonal coordinates for elements of $E_{n}$ and consider $W\left(C_{n}\right)$-orbits $O(\lambda)$ with $\lambda=\left(m_{1}, m_{2}, \ldots, m_{n}\right)$ such that $m_{1}>m_{2}>\cdots>m_{n}>0$. The orbit $O(\lambda)$ consists of all points (4.10). Restriction of these points to the vector subspace $E_{p} \times E_{q}$ reduces to splitting the set of coordinates (4.10) into two parts:

$$
\left( \pm m_{i_{1}}, \pm m_{i_{2}}, \ldots, \pm m_{i_{p}}\right)\left( \pm m_{i_{p+1}}, \ldots, \pm m_{i_{n}}\right) .
$$

Due to the condition $m_{1}>m_{2}>\cdots>m_{n}>0$, these elements do not lie on walls of the $W\left(A_{p-1}\right) \times W\left(C_{q}\right)$-chambers. We have to choose dominant elements (with respect to the Weyl group $\left.W\left(A_{p-1}\right) \times W\left(C_{q}\right)\right)$ in the set of points (4.12). The conditions for elements of $E_{p}$ and $E_{q}$ to be dominant implies that only the elements

$$
\left(m_{i_{1}}, \ldots, m_{i_{j}},-m_{i_{j+1}}, \ldots,-m_{i_{p}}\right)\left(m_{i_{p+1}}, \ldots, m_{i_{n}}\right), \quad j=0,1,2, \ldots, p,
$$

satisfying

$$
m_{i_{1}}>m_{i_{2}}>\cdots>m_{i_{j}}, \quad m_{i_{j+1}}<m_{i_{j+2}}<\cdots<m_{i_{p}}, \quad m_{i_{p+1}}>m_{i_{p+2}}>\cdots>m_{i_{n}},
$$

are dominant. Moreover, each such point is contained in the $W\left(C_{n}\right)$-orbit $O(\lambda)$ only once. This assertion completely determines the list of $W\left(A_{p-1}\right) \times W\left(C_{q}\right)$-orbits in $W\left(C_{n}\right)$-orbit $O(\lambda)$. All $W\left(A_{p-1}\right) \times W\left(C_{q}\right)$-orbits are contained in $O(\lambda)$ with multiplicity 1 .

\subsection{Decomposition of $W\left(D_{n}\right)$-orbits into $W\left(D_{n-1}\right)$-orbits}

If $\alpha_{1}, \alpha_{2}, \ldots, \alpha_{n}$ is the set of simple roots of $D_{n}$, then the roots $\alpha_{2}, \ldots, \alpha_{n}$ are simple roots of $D_{n-1}$. The last roots span the subspace $E_{n-1}$. It is assumed that $n>4$. 
For elements $\lambda$ of $E_{n}$ we again use orthogonal coordinates $m_{1}, m_{2}, \ldots, m_{n}$. Then $\lambda$ is dominant if $m_{1} \geq m_{2} \geq \cdots \geq m_{n-1} \geq\left|m_{n}\right|$. We assume that $\lambda$ satisfies the condition

$$
m_{1}>m_{2}>\cdots>m_{n}>0
$$

that is, $\lambda$ does not lie on a wall of the dominant Weyl chamber. Then the orbit $O(\lambda)$ consists of all points

$$
w\left(m_{1}, m_{2}, \ldots, m_{n}\right)=\left( \pm m_{i_{1}}, \pm m_{i_{2}}, \ldots, \pm m_{i_{n}}\right), \quad w \in W\left(D_{n}\right),
$$

where $\left(i_{1}, i_{2}, \ldots, i_{n}\right)$ is a permutation of the numbers $1,2, \ldots, n$ and there is an even number of minus signs. Restricting the elements of (4.13) to the subspace $E_{n-1}$ is reduced to removing the first coordinate $\pm m_{i_{1}}$ in (4.13). As a result, we obtain from the set of points (4.13) the collection

$$
\left( \pm m_{i_{2}}, \pm m_{i_{3}}, \ldots, \pm m_{i_{n}}\right), \quad w \in W\left(D_{n}\right)
$$

where the number of minus signs may either be even or odd, depending on a sign of the removed number $\pm m_{i_{1}}$. Only the points of the form $\left(m_{i_{2}}, m_{i_{3}}, \ldots, m_{i_{n-1}}, \pm m_{i_{n}}\right)$ may be dominant. Moreover, such a point is dominant if and only if

$$
m_{i_{2}}>m_{i_{3}}>\cdots>m_{i_{n}}>0
$$

Thus, if we restrict the points (4.13) to $E_{n-1}$ we obtain the following $W\left(D_{n-1}\right)$-dominant elements:

$$
\left(m_{1}, m_{2}, \ldots, m_{i-1}, \hat{m}_{i}, m_{i+1}, \ldots, \pm m_{n}\right), \quad i=1,2, \ldots, n
$$

where a hat over $m_{i}$ means that the coordinate $m_{i}$ must be omitted (if $i=n$ then, instead of $\pm m_{n}$, we have to take $\pm m_{n-1}$ in (4.14)). Moreover, the element (4.14) with fixed $i$ can be obtained only from one element in (4.13), namely, from element $\left(m_{1}, m_{2}, \ldots, m_{i-1}, \pm m_{i}, m_{i+1}, \ldots\right.$, $\left.\pm m_{n}\right)$, where at $m_{i}$ and $m_{n}$ a sign is the same.

Thus, the $W\left(D_{n}\right)$-orbits $O\left(m_{1}, m_{2}, \ldots, m_{n}\right)$ with $m_{1}>m_{2}>\cdots>m_{n}>0$ consists of the following $W\left(D_{n-1}\right)$-orbits:

$$
O\left(m_{1}, m_{2}, \ldots, m_{i-1}, \hat{m}_{i}, m_{i+1}, \ldots, \pm m_{n}\right), \quad i=1,2, \ldots, n .
$$

Each such $W\left(D_{n-1}\right)$-orbit is contained in $O\left(m_{1}, m_{2}, \ldots, m_{n}\right)$ with multiplicity 1.

It is shown similarly that the $W\left(D_{n}\right)$-orbits

$$
O\left(m_{1}, m_{2}, \ldots, m_{n-1},-m_{n}\right), \quad m_{1}>m_{2}>\cdots>m_{n}>0
$$

consists of the same $W\left(D_{n-1}\right)$-orbits as the $W\left(D_{n}\right)$-orbits $O\left(m_{1}, \ldots, m_{n-1}, m_{n}\right)$ with the same set of numbers $m_{1}, m_{2}, \ldots, m_{n}$.

\subsection{Decomposition of $W\left(D_{n}\right)$-orbits into $W\left(A_{p-1}\right) \times W\left(D_{q}\right)$-orbits, $p+q=n, q \geq 4$}

If $\alpha_{1}, \alpha_{2}, \ldots, \alpha_{n}$ are simple roots of $D_{n}$, then $\alpha_{1}, \alpha_{2}, \ldots, \alpha_{p-1}$ are simple roots of $A_{p-1}$ (they can be embedded into the linear subspace $E_{p}$ ) and $\alpha_{p+1}, \alpha_{p+2}, \ldots, \alpha_{n}$ are simple roots of $D_{q}$ (they generate the linear subspace $E_{q}$ ).

We use orthogonal coordinates in $E_{n}$ and consider $W\left(D_{n}\right)$-orbits $O(\lambda)$ with an element $\lambda=\left(m_{1}, m_{2}, \ldots, m_{n}\right)$ such that $m_{1}>m_{2}>\cdots>m_{n}>0$. The orbit $O(\lambda)$ consists of all 
points (4.13). Restricting these points to the vector subspace $E_{p} \times E_{q}$ is reduced to splitting the set of coordinates (4.13) into two parts:

$$
\left( \pm m_{i_{1}}, \pm m_{i_{2}}, \ldots, \pm m_{i_{p}}\right)\left( \pm m_{i_{p+1}}, \ldots, \pm m_{i_{n}}\right) .
$$

Due to the condition $m_{1}>m_{2}>\cdots>m_{n}>0$, these elements do not lie on walls of the dominant $W\left(A_{p-1}\right) \times W\left(D_{q}\right)$-chamber. We have to choose dominant elements (with respect to the Weyl group $\left.W\left(A_{p-1}\right) \times W\left(D_{q}\right)\right)$ in the set of points (4.15). The conditions for elements of $E_{p}$ and $E_{q}$ to be dominant implies that the elements

$$
\left(m_{i_{1}}, \ldots, m_{i_{j}},-m_{i_{j+1}}, \ldots,-m_{i_{p}}\right)\left(m_{i_{p+1}}, \ldots, \pm m_{i_{n}}\right), \quad j=0,1,2, \ldots, p
$$

satisfying the conditions

$$
m_{i_{1}}>m_{i_{2}}>\cdots>m_{i_{j}}, \quad m_{i_{j+1}}<m_{i_{j+2}}<\cdots<m_{i_{p}}, \quad m_{i_{p+1}}>m_{i_{p+2}}>\cdots>m_{i_{n}},
$$

are dominant if and only if the number of minus signs is even. Moreover, each such point is contained in the $W\left(D_{n}\right)$-orbit $O(\lambda)$ only once. These assertions completely determine the list of $W\left(A_{p-1}\right) \times W\left(D_{q}\right)$-orbits in $W\left(D_{n}\right)$-orbit $O(\lambda)$. All $W\left(A_{p-1}\right) \times W\left(D_{q}\right)$-orbits are contained in $O(\lambda)$ with multiplicity 1.

\subsection{Decomposition by means of a projection matrix}

In above examples of decomposition of $W$-orbits into $W^{\prime}$-orbits, the $W^{\prime}$-system of simple roots was a subsystem of $W$-system of simple roots. The present case is simple and the decomposition is easily fulfilled.

There exist much more complicated cases of the embeddings $W^{\prime} \subset W$ (and of the embeddings $L^{\prime} \subset L$ of the corresponding Lie algebras). In these cases, the collection of the unit vectors $\omega_{1}^{\prime}, \omega_{2}^{\prime}, \ldots, \omega_{m}^{\prime}$ (they are such that $2\left\langle\omega_{i}^{\prime}, \alpha_{j}^{\prime}\right\rangle /\left\langle\alpha_{j}^{\prime}, \alpha_{j}^{\prime}\right\rangle=\delta_{i j}$ for simple roots $\alpha_{1}^{\prime}, \alpha_{2}^{\prime}, \ldots, \alpha_{m}^{\prime}$ corresponding to the Weyl group $W^{\prime}$ ) is not a subsystem of the unit vectors $\omega_{1}, \omega_{2}, \ldots, \omega_{n}$ (for which $2\left\langle\omega_{i}, \alpha_{j}\right\rangle /\left\langle\alpha_{j}, \alpha_{j}\right\rangle=\delta_{i j}$ for simple roots $\alpha_{1}, \alpha_{2}, \ldots, \alpha_{n}$ corresponding to the Weyl group $W)$. This fact make the decomposition procedure complicated.

In these cases a projection matrix, constructed for a fixed pair $W^{\prime} \subset W$ and projecting weights $\lambda=\left(\lambda_{1}, \lambda_{2}, \ldots, \lambda_{n}\right)$ in the $\omega$-coordinates to weights in the $\omega^{\prime}$-coordinates (corresponding to the Weyl subgroup $\left.W^{\prime}\right)$, is used. Such a matrix is of dimension $m \times n$, where $m$ is the number of $\omega^{\prime}$-coordinates. A list of such matrices for a large number of pairs $W^{\prime} \subset W$ is given in [27].

When performing the decomposition of a $W$-orbit $O(\lambda)$ into $W^{\prime}$-orbits we have to project $\omega$-coordinates of all weights of $O(\lambda)$ to $\omega^{\prime}$-coordinates and then we have to split the points obtained under this projection into $W^{\prime}$-orbits.

Let us give an explicit form of the projection matrices for some simple cases:

$$
\begin{aligned}
& C_{2} \rightarrow A_{1}:\left(\begin{array}{ll}
3 & 4
\end{array}\right) ; \quad G_{2} \rightarrow A_{1}:\left(\begin{array}{ll}
10 & 6
\end{array}\right) ; \\
& C_{2} \rightarrow A_{1} \times A_{1}:\left(\begin{array}{cc}
1 & 1 \\
0 & 1
\end{array}\right) ; \quad G_{2} \rightarrow A_{2}:\left(\begin{array}{cc}
1 & 1 \\
1 & 0
\end{array}\right) ; \\
& C_{4} \rightarrow A_{3}:\left(\begin{array}{cccc}
1 & 1 & 0 & 0 \\
0 & 0 & 1 & 2 \\
0 & 1 & 1 & 0
\end{array}\right) ; \quad D_{5} \rightarrow C_{2} \times C_{2}:\left(\begin{array}{ccccc}
0 & 0 & 2 & 1 & 1 \\
1 & 1 & 0 & 0 & 0 \\
0 & 0 & 0 & 1 & 1 \\
0 & 1 & 1 & 0 & 0
\end{array}\right) .
\end{aligned}
$$




\subsection{Generating functions for multiplicities}

Multiplicities of orbits in products of orbits and in the restriction of $W$-orbits into $W^{\prime}$-orbits can be derived by means of generating functions for these multiplicities. A series of such generating functions are derived in [28] and [29]. Let us give some examples in this subsection.

First we consider the restriction $B_{3} \rightarrow B_{2} \times U(1)$, where it is supposed that an orbit of $U(1)$ is given by one integer or half-integer. The generating function for this case is

$$
\begin{aligned}
& \frac{1}{\left(1-a_{1}\right)\left(1-c_{2}\right)\left(1-e_{3}\right)}+\frac{e_{3}^{*}}{\left(1-a_{1}\right)\left(1-c_{2}\right)\left(1-e_{3}^{*}\right)}+\frac{d_{2}}{\left(1-a_{1}\right)\left(1-d_{2}\right)\left(1-e_{3}\right)} \\
& +\frac{b_{2}^{*}}{\left(1-a_{1}\right)\left(1-d_{2}^{*}\right)\left(1-e_{3}^{*}\right)}+\frac{b_{1}^{*}}{\left(1-b_{1}\right)\left(1-d_{2}\right)\left(1-e_{3}\right)}+\frac{\left.d_{1}^{*}\right)\left(1-d_{2}^{*}\right)\left(1-e_{3}^{*}\right)}{\left(1-b_{1}^{*}\right)}
\end{aligned}
$$

where

$$
\begin{aligned}
& a_{1}=A_{1} B_{1}, \quad b_{1}=A_{1} Z, \quad b_{1}^{*}=A_{1} Z^{-1}, \quad c_{2}=A_{2} B_{2}^{2}, \\
& d_{2}=A_{2} B_{1} Z, \quad d_{2}^{*}=A_{2} B_{1} Z^{-1}, \quad e_{3}=A_{3} B_{2} Z^{1 / 2}, \quad e_{3}^{*}=A_{3} B_{2} Z^{-1 / 2} .
\end{aligned}
$$

The multiplicities in this case are 0 or 1 . In order to obtain a multiplicity of a $B_{2} \times U(1)$-orbit $O\left(\mu_{1}, \mu_{2} ; z\right)$ in a $B_{3}$-orbit $O\left(\lambda_{1}, \lambda_{2}, \lambda_{3}\right)$ (the numbers $\lambda_{1}, \lambda_{2}, \lambda_{3}$ and $\mu_{1}, \mu_{2}$ give $\omega$-coordinates of the corresponding elements) we have to expand (4.16) into a power series. The multiplicity is given by the term $A_{1}^{\lambda_{1}} A_{2}^{\lambda_{2}} A_{3}^{\lambda_{3}} B_{1}^{\mu_{1}} B_{2}^{\mu_{2}} Z^{z}$ in the expansion: if this term is absent then the multiplicity is 0 ; if it exists (i.e. a coefficient of it is equal to 1 ) then the multiplicity is 1 . Note that multiplicities in the restriction $B_{3} \rightarrow B_{2} \times U(1)$ trivially determine multiplicities in the restriction $B_{3} \rightarrow B_{2}$.

Multiplicities of $A_{2}$-orbits in $G_{2}$-orbits are given in the same way by the generating function

$$
\frac{1}{1-a_{1}}\left[\frac{1}{1-b_{2}}+\frac{b_{2}^{*}}{1-b_{2}^{*}}\right]
$$

where

$$
a_{1}=A_{1} B_{1} B_{2}, \quad b_{2}=A_{2} B_{1}, \quad b_{2}^{*}=A_{2} B_{2}
$$

and the symbols $A_{1}, A_{2}$ and $B_{1}, B_{2}$ are related to $G_{2}$-orbits and $A_{2}$-orbits, respectively.

Multiplicities of $B_{3} \times A_{1}$-orbits in $F_{4}$-orbits are given by the generating function

$$
\begin{aligned}
\frac{1}{\left(1-a_{1}\right)\left(1-e_{2}\right)\left(1-h_{3}\right)\left(1-k_{4}\right)}+\frac{i_{3}}{\left(1-a_{1}\right)\left(1-e_{2}\right)\left(1-i_{3}\right)\left(1-k_{4}\right)} \\
\quad+\frac{l_{4}}{\left(1-a_{1}\right)\left(1-e_{2}\right)\left(1-i_{3}\right)\left(1-l_{4}\right)}+\frac{b_{1}}{\left(1-b_{1}\right)\left(1-e_{2}\right)\left(1-h_{3}\right)\left(1-k_{4}\right)} \\
\quad+\frac{f_{2}}{\left(1-b_{1}\right)\left(1-d_{2}\right)\left(1-h_{3}\right)\left(1-k_{4}\right)}+\frac{f_{3}}{\left(1-b_{1}\right)\left(1-f_{2}\right)\left(1-i_{3}\right)\left(1-l_{4}\right)} \\
\quad+\frac{g_{2}}{\left(1-b_{1}\right)\left(1-f_{2}\right)\left(1-j_{3}\right)\left(1-l_{4}\right)}+\frac{b_{1} i_{3}}{\left(1-b_{1}\right)\left(1-g_{2}\right)\left(1-j_{3}\right)\left(1-l_{4}\right)} \\
\quad+\frac{b_{1} l_{4}}{\left(1-b_{1}\right)\left(1-e_{2}\right)\left(1-i_{3}\right)\left(1-k_{4}\right)}+\frac{c_{2} k_{4}}{\left(1-b_{1}\right)\left(1-e_{2}\right)\left(1-i_{3}\right)\left(1-l_{4}\right)} \\
\quad+\frac{c_{1}}{\left(1-b_{1}\right)\left(1-f_{2}\right)\left(1-i_{3}\right)\left(1-k_{4}\right)}+\frac{\left.c_{2}\right)\left(1-g_{2}\right)\left(1-j_{3}\right)\left(1-l_{4}\right)}{\left(1-c_{1}\right)}
\end{aligned}
$$

where

$$
a_{1}=A_{1} B_{1}^{2}, \quad b_{1}=A_{1} B_{3}^{2} C, \quad c_{1}=A_{1} C^{2}, \quad d_{2}=A_{2} B_{3}^{4}, \quad e_{2}=A_{2} B_{1}^{2} B_{2}^{2} C,
$$




$$
\begin{array}{ll}
f_{2}=A_{2} B_{2}^{2} C^{2}, & g_{2}=A_{2} B_{3}^{2} C^{3}, \quad h_{3}=A_{3} B_{1} B_{3}^{2}, \quad i_{3}=A_{3} B_{1} B_{2} C, \\
j_{3}=A_{3} B_{2} C^{2}, & k_{4}=A_{4} B_{2}, \quad l_{4}=A_{4} B_{1} C .
\end{array}
$$

and the symbols $A_{1}, A_{2}, A_{3}, A_{4} ; B_{1}, B_{2}, B_{3}$ and $C$ are related to $F_{4^{-}}, B_{3^{-}}$and $A_{1^{-} \text {-orbits, }}$ respectively.

\section{Affine Weyl group and its fundamental domain}

\subsection{Affine Weyl groups}

We are interested in orbit functions which are given on the Euclidean space $E_{n}$. Orbit functions are invariant with respect to action of a Weyl group $W$, which is a transformation group of $E_{n}$. However, $W$ does not describe all symmetries of orbit functions. A whole group of invariances of orbit functions is isomorphic to the so called affine Weyl group $W^{\text {aff }}$ which is an extension of the Weyl group $W$. In this subsection we define affine Weyl groups.

Let $\alpha_{1}, \alpha_{2}, \ldots, \alpha_{n}$ be simple roots in the Euclidean space $E_{n}$ and let $W$ be the corresponding Weyl group. The group $W$ is generated by the reflections $r_{\alpha_{i}}, i=1,2, \ldots, n$, corresponding to simple roots. We consider also the reflection $r_{\xi}$ with respect to the $(n-1)$-dimensional subspace (hyperplane) $X_{n-1}$ containing the origin and orthogonal to the highest (long) root $\xi$, given in (2.8):

$$
r_{\xi} x=x-\frac{2\langle x, \xi\rangle}{\langle\xi, \xi\rangle} \xi
$$

We shift the hyperplane $X_{n-1}$ by the vector ${ }^{1} \xi^{\vee} / 2$, where $\xi^{\vee}=2 \xi /\langle\xi, \xi\rangle$. The reflection with respect to the hyperplane $X_{n-1}+\xi^{\vee} / 2$ will be denoted by $r_{0}$. Then in order to fulfill the transformation $r_{0}$ we have to fulfill the transformation $r_{\xi}$ and then to shift the result by $\xi^{\vee}$, that is,

$$
r_{0} x=r_{\xi} x+\xi^{\vee} .
$$

We have $r_{0} 0=\xi^{\vee}$ and it follows from (5.1) that $r_{0}$ maps $x+\xi^{\vee} / 2$ to

$$
r_{\xi}\left(x+\xi^{\vee} / 2\right)+\xi^{\vee}=x+\xi^{\vee} / 2-\left\langle x, \xi^{\vee}\right\rangle \xi .
$$

Therefore,

$$
\begin{aligned}
r_{0}\left(x+\xi^{\vee} / 2\right) & =x+\xi^{\vee} / 2-\frac{2\langle x, \xi\rangle}{\langle\xi, \xi\rangle} \xi=x+\xi^{\vee} / 2-\frac{2\left\langle x, \xi^{\vee}\right\rangle}{\left\langle\xi^{\vee}, \xi^{\vee}\right\rangle} \xi^{\vee} \\
& =x+\xi^{\vee} / 2-\frac{2\left\langle x+\xi^{\vee} / 2, \xi^{\vee}\right\rangle}{\left\langle\xi^{\vee}, \xi^{\vee}\right\rangle} \xi^{\vee}+\frac{2\left\langle\xi^{\vee} / 2, \xi^{\vee}\right\rangle}{\left\langle\xi^{\vee}, \xi^{\vee}\right\rangle} \xi^{\vee} .
\end{aligned}
$$

Denoting $x+\xi^{\vee} / 2$ by $y$ we obtain that $r_{0}$ is given also by the formula

$$
r_{0} y=y+\left(1-\frac{2\left\langle y, \xi^{\vee}\right\rangle}{\left\langle\xi^{\vee}, \xi^{\vee}\right\rangle}\right) \xi^{\vee}=\xi^{\vee}+r_{\xi} y .
$$

The hyperplane $X_{n-1}+\xi^{\vee} / 2$ coincides with the set of points $y$ such that $r_{0} y=y$. It follows from (5.2) that this hyperplane is given by the equation

$$
1=\frac{2\left\langle y, \xi^{\vee}\right\rangle}{\left\langle\xi^{\vee}, \xi^{\vee}\right\rangle}=\langle y, \xi\rangle=\sum_{k=1}^{n} a_{k} q_{k},
$$

\footnotetext{
${ }^{1}$ Note that under our conditions for lengths of roots in Subsection 2.3 we have $\xi^{\vee}=\xi$.
} 
where

$$
y=\sum_{k=1}^{n} a_{k} \omega_{k}, \quad \xi=\sum_{k=1}^{n} q_{k} \alpha_{k}^{\vee}
$$

(see (2.4)). In Subsection 2.3, the numbers $q_{i}$ are given in an explicit form.

A group of transformations of the Euclidean space $E_{n}$ generated by the reflections $r_{0}, r_{\alpha_{1}}, \ldots$, $r_{\alpha_{n}}$ is called the affine Weyl group of the root system $R$ and is denoted by $W^{\text {aff }}$ or by $W_{R}^{\text {aff }}$ (if is necessary to indicate the initial root system).

\subsection{Properties of affine Weyl groups}

Adjoining the reflection $r_{0}$ to the Weyl group $W$ completely changes the properties of the group $W^{\text {aff }}$.

If $r_{\xi}$ is the reflection with respect to the hyperplane $X_{n-1}$, then due to (5.2) for any $x \in E_{n}$ we have

$$
r_{0} r_{\xi} x=r_{0}\left(r_{\xi} x\right)=\xi^{\vee}+r_{\xi} r_{\xi} x=x+\xi^{\vee} .
$$

Clearly, $\left(r_{0} r_{\xi}\right)^{k} x=x+k \xi^{\vee}, k=0, \pm 1, \pm 2, \ldots$, that is, the set of elements $\left(r_{0} r_{\xi}\right)^{k}, k=$ $0, \pm 1, \pm 2, \ldots$, is an infinite commutative subgroup of $W^{\text {aff }}$. This means that (unlike to the Weyl group $W) W^{\text {aff }}$ is an infinite group.

Recall that $r_{0} 0=\xi^{\vee}$. For any $w \in W$ we have

$$
w r_{0} 0=w \xi^{\vee}=\xi_{w}^{\vee}
$$

where $\xi_{w}^{\vee}$ is a coroot of the same length as the coroot $\xi^{\vee}$. For this reason, $w r_{0}$ is the reflection with respect to the $(n-1)$-hyperplane perpendicular to the root $\xi_{w}^{\vee}$ and containing the point $\xi_{w}^{\vee} / 2$. Moreover,

$$
\left(w r_{0}\right) r_{\xi_{w}} x=x+\xi_{w}^{\vee}
$$

We also have $\left(\left(w r_{0}\right) r_{\xi_{w}}\right)^{k} x=x+k \xi_{w}^{\vee}, k=0, \pm 1, \pm 2, \ldots$. Since $w$ is any element of $W$, then the set $w \xi^{\vee}, w \in W$, coincides with the set of coroots of $R^{\vee}$, corresponding to all long roots of root system $R$. Thus, the following proposition is true:

Proposition 5. The set $W^{\text {aff }} \cdot 0$ coincides with the lattice $Q_{l}^{\vee}$ generated by coroots $\alpha^{\vee}$ taken for all long roots $\alpha$ from $R$.

It is easy to see from explicit forms of the root systems $Q$ and $Q^{\vee}$ that each coroot $\xi_{s}^{\vee}$ for a short root $\xi_{s}$ of $R$ is a linear combination of coroots $w \xi^{\vee} \equiv \xi_{w}, w \in W$, with integral coefficients, that is, $Q^{\vee}=Q_{l}^{\vee}$. Therefore, from Proposition 5 we obtain the following corollary:

Corollary. The set $W^{\text {aff }} \cdot 0$ coincides with the coroot lattice $Q^{\vee}$ of $R$.

Let $\hat{Q}^{\vee}$ be the subgroup of $W^{\text {aff }}$ generated by the elements

$$
\left(w r_{0}\right) r_{w}, \quad w \in W
$$

where $r_{w} \equiv r_{\xi_{w}}$ for $w \in W$ (see (5.4)). Since elements (5.5) pairwise commute with each other (since they are shifts), $\hat{Q}^{\vee}$ is a commutative group. The subgroup $\hat{Q}^{\vee}$ can be identified with the coroot lattice $Q^{\vee}$. Namely, if for $g \in \hat{Q}^{\vee}$ we have $g \cdot 0=\gamma \in Q^{\vee}$ (that is, $g$ is a shift by $\gamma$ ), then $g$ is identified with $\gamma$. This correspondence is one-to-one.

The subgroups $W$ and $\hat{Q}^{\vee}$ generate $W^{\text {aff }}$ since a subgroup of $W^{\text {aff }}$, generated by $W$ and $\hat{Q}^{\vee}$, contains the element $r_{0}$. From the other side, $W \cup \hat{Q}^{\vee}=\{1\}$, since $W$ does not contain shifts. 
Moreover, $\hat{Q}^{\vee}$ is an invariant subgroup of $W^{\text {aff }}$ since for any element $\left(w r_{0}\right) r_{w}$ from (5.5) and for any element $w^{\prime} \in W$ we have

$$
w^{\prime}\left(w r_{0} r_{w}\right) w^{\prime-1} x=w^{\prime}\left(w r_{0} r_{w}\right)\left(w^{\prime-1} x\right)=w^{\prime}\left(w^{\prime-1} x+\xi_{w}^{\vee}\right)=x+w^{\prime} \xi_{w}^{\vee},
$$

where $w^{\prime} \xi_{w}^{\vee} \in Q^{\vee}$, that is, $w^{\prime}\left(w r_{0} r_{w}\right) w^{\prime-1}$ is an element $g^{\prime}$ of $\hat{Q}^{\vee}$ such that $g^{\prime} x=x+w^{\prime} \xi_{w}^{\vee}$. Thus, we have proved the following proposition:

Proposition 6. The group $W^{\text {aff }}$ is a semidirect product of its subgroups $W$ and $\hat{Q}^{\vee}$, where $\hat{Q}^{\vee}$ is an invariant subgroup.

It follows from this proposition that each element of $W^{\text {aff }}$ can be represented as a product $w g$, $w \in W, g \in \hat{Q}^{\vee}$, or as a product $g w, w \in W, g \in \hat{Q}^{\vee}$.

\subsection{Fundamental domain}

An open connected simply connected set $D \subset E_{n}$ is called a fundamental domain for the group $W^{\text {aff }}$ (for the group $W$ ) if it does not contain equivalent points (that is, points $x$ and $x^{\prime}$ such that $x^{\prime}=w x$ ) and if its closure contains at least one point from each $W^{\text {aff }}$-orbit (from each $W$-orbit). It is evident that the dominant Weyl chamber (without the walls of this chamber) is a fundamental domain for the Weyl group $W$. Recall that this domain consists of all points $x=a_{1} \omega_{1}+\cdots+a_{n} \omega_{n} \in E_{n}$ for which

$$
a_{i}=\left\langle x, \alpha_{i}^{\vee}\right\rangle>0, \quad i=1,2, \ldots, n .
$$

Let us describe the fundamental domain of the group $W^{\text {aff }}$. Since $W \subset W^{\text {aff }}$, it can be chosen as a subset of the dominant Weyl chamber of $W$.

We have seen that the element $r_{0} \in W^{\text {aff }}$ is a reflection with respect to the hyperplane $X_{n-1}+\xi^{\vee} / 2$, orthogonal to the root $\xi$ and containing the point $\xi^{\vee} / 2$. This hyperplane is given by the equation (5.3). This equation shows that the hyperplane $X_{n-1}+\xi^{\vee} / 2$ intersects the axes, determined by the orbits $\omega_{i}$, in the points $\omega_{i} / q_{i}, i=1,2, \ldots, n$, where $q_{i}$ are such as in (5.3). We create the simplex with $n+1$ vertices in the points

$$
0, \frac{\omega_{1}}{q_{1}}, \ldots, \frac{\omega_{n}}{q_{n}}
$$

By the definition, this simplex consists of all points $y$ of the dominant Weyl chamber for which $\langle y, \xi\rangle \leq 1$. Clearly, the interior $F$ of this simplex belongs to the dominant Weyl chamber.

Theorem 1. The set $F$ is a fundamental domain for the affine Weyl group $W^{\text {aff }}$.

Proof. The set $\bar{F}$ coincides with the set of points $y$ of the dominant Weyl chamber, for which $\langle y, \xi\rangle \leq 1$. Then

$$
\langle y, \alpha\rangle \leq 1, \quad \alpha \in R_{l},
$$

where $R_{l}$ is a subset of the root set $R$ consisting of all long roots. Indeed, for any $\alpha \in R_{l}$ we have $\alpha=\xi-\sum_{i} a_{i} \alpha_{i}$, where $\alpha_{i}$ are simple roots and $a_{i}$ are non-negative integers. Then $\langle y, \alpha\rangle=\langle y, \xi\rangle-\sum_{i} a_{i}\left\langle y, \alpha_{i}\right\rangle \leq 1$, since $\left\langle y, \alpha_{i}\right\rangle \geq 0$ for all $y \in \bar{F}$. Acting upon $\bar{F}$ by elements of the Weyl group $W$ we obtain the domain $\mathcal{D} \subset E_{n}$ determined by the equations

$$
\langle y, \alpha\rangle \leq 1, \quad \alpha \in R_{l} .
$$

The hyperplanes $\langle y, \alpha\rangle=1, \alpha \in R_{l}$, determine walls of the domain $\mathcal{D}$. Since $\left\langle\alpha^{\vee}, \alpha\right\rangle=2$, for each fixed root $\alpha \in R_{l}$ a distance between the opposite hyperplanes $\langle y, \alpha\rangle=1$ and $\langle y,-\alpha\rangle=1$ 
is $\alpha^{\vee}$. Thus, nontrivial elements of $W$ do not map elements of $F$ inside of $F$ and nontrivial elements of $\hat{Q}^{\vee}$ do not map elements of $\mathcal{D}$ inside of $\mathcal{D}$. From the other side, successive shifts of $\mathcal{D}$ by coroots cover the whole space $E_{n}$.

Thus, we have $\cup_{w \in W \text { aff }} w \bar{F}=E_{n}$. Clearly, we cannot remove from $F$ any point $x$ since then $F$ is not simply connected. Therefore, $F$ is a fundamental domain for the group $W^{\text {aff }}$. The theorem is proved.

For the rank 2 cases the fundamental domain is a simplex with the following vertices:

$$
\begin{aligned}
& A_{2}:\left\{0, \omega_{1}, \omega_{2}\right\}, \\
& C_{2}:\left\{0, \omega_{1}, \omega_{2}\right\}, \\
& G_{2}:\left\{0, \frac{\omega_{1}}{2}, \omega_{2}\right\} .
\end{aligned}
$$

\section{Orbit functions}

\subsection{Definition}

The exponential functions $e^{2 \pi \mathrm{i}\langle m, x\rangle}, x \in E_{n}$, with fixed $m=\left(m_{1}, m_{2}, \ldots, m_{n}\right), m_{i} \in \mathbb{R}$, determine the Fourier transform on $E_{n}$. Orbit functions are a symmetrized (with respect to a Weyl group) version of exponential functions. Correspondingly, they determine a symmetrized version of the Fourier transform.

Orbit functions are defined as follows. Let $W$ be a Weyl group of transformations of the Euclidean space $E_{n}$. To each element $\lambda \in E_{n}$ from the dominant Weyl chamber (including its walls) there corresponds an orbit function $\phi_{\lambda}$ on $E_{n}$, which is given by the formula

$$
\phi_{\lambda}(x)=\sum_{\mu \in O(\lambda)} e^{2 \pi \mathrm{i}\langle\mu, x\rangle}, \quad x \in E_{n}
$$

where $O(\lambda)$ is the $W$-orbit of the element $\lambda$. It is also called a C-function (since for the case $A_{1}$ it coincides with the cosine). The number of summands in (6.1) is equal to the size $|O(\lambda)|$ of the orbit $O(\lambda)$ which coincides with the number $|W| /\left|W_{\lambda}\right|$. Clearly, $\phi_{\lambda}(0)=|W| /\left|W_{\lambda}\right|$.

Sometimes (see, for example, [30] and [31]), it is convenient to use a modified definition of orbit functions:

$$
\hat{\phi}_{\lambda}(x)=\left|W_{\lambda}\right| \phi_{\lambda}(x) .
$$

Then for all orbit functions $\hat{\phi}_{\lambda}$ we have $\hat{\phi}_{\lambda}(0)=|W|$.

We are mainly interested in orbit functions $\phi_{\lambda}$ for which $\lambda \in P_{+}$. Namely, such orbit functions determine a symmetrized Fourier series expansion which will be studied in the next section.

Example: Orbit functions for $A_{1}$. In this case, there exists only one simple (positive) root $\alpha$. We have $\langle\alpha, \alpha\rangle=2$. Therefore, the relation $2\langle\omega, \alpha\rangle /\langle\alpha, \alpha\rangle=1$ means that $\langle\omega, \alpha\rangle=1$. This means that $\omega=\alpha / 2$ and $\langle\omega, \omega\rangle=1 / 2$. Elements of $P_{+}$coincide with $m \omega, m \in \mathbb{Z}_{+}$. We identify points $x$ of $E_{1} \equiv \mathbb{R}$ with $\theta \omega$. Since the Weyl group $W\left(A_{1}\right)$ consists of two elements 1 and $r_{\alpha}$, and

$$
r_{\alpha} x=x-\frac{2\langle\theta \omega, \alpha\rangle}{\langle\alpha, \alpha\rangle} \alpha=x-\theta \alpha=x-2 x=-x,
$$

orbit functions $\phi_{\lambda}(x), \lambda=m \omega$, are given by the formula

$$
\phi_{\lambda}(x)=e^{2 \pi \mathrm{i}\langle m \omega, \theta \omega\rangle}+e^{2 \pi \mathrm{i}\langle m \omega,-\theta \omega\rangle}=e^{\pi \mathrm{i} m \theta}+e^{-\pi \mathrm{i} m \theta}=2 \cos (\pi m \theta) \text {, }
$$

where $m \neq 0$. If $m=0$, then $\phi_{\lambda}(x) \equiv 1$. 


\subsection{Orbit functions of $A_{2}$}

Let $\lambda=a \omega_{1}+b \omega_{2} \equiv(a b)$, with $a>b>0$. Then taking into account the results of Subsection 2.5, for $\phi_{\lambda}(x) \equiv \phi_{(a b)}(x)$ we have that

$$
\begin{aligned}
\phi_{(a b)}(x)= & e^{2 \pi i\langle(a b), x\rangle}+e^{2 \pi i\langle(-a a+b), x\rangle}+e^{2 \pi i\langle(a+b-b), x\rangle} \\
& +e^{2 \pi i\langle(b-a-b), x\rangle}+e^{2 \pi i\langle(-a-b a), x\rangle}+e^{2 \pi i\langle(-b-a), x\rangle} .
\end{aligned}
$$

Using the representation $x=\varphi_{1} \alpha_{1}^{\vee}+\varphi_{2} \alpha_{2}^{\vee}$, one obtains

$$
\begin{aligned}
\phi_{(a b)}(x)= & e^{2 \pi i\left(a \varphi_{1}+b \varphi_{2}\right)}+e^{2 \pi i\left(-a \varphi_{1}+(a+b) \varphi_{2}\right)}+e^{2 \pi i\left((a+b) \varphi_{1}-b \varphi_{2}\right)} \\
& +e^{2 \pi i\left(b \varphi_{1}-(a+b) \varphi_{2}\right)}+e^{2 \pi i\left((-a-b) \varphi_{1}+a \varphi_{2}\right)}+e^{2 \pi i\left(-b \varphi_{1}-a \varphi_{2}\right)} .
\end{aligned}
$$

The actual expression for $\phi_{(a, b)}(x)$ depends on the choice of coordinate systems for $\lambda$ and $x$. Setting $x=\theta_{1} \omega_{1}+\theta_{2} \omega_{2}$ and $\lambda$ as before, after using formula (2.6) we get

$$
\begin{aligned}
\phi_{(a b)}(x)= & e^{\frac{2 \pi i}{3}\left((2 a+b) \theta_{1}+(a+2 b) \theta_{2}\right)}+e^{\frac{2 \pi i}{3}\left((-a+b) \theta_{1}+(a+2 b) \theta_{2}\right)} \\
& +e^{\frac{2 \pi i}{3}\left((2 a+b) \theta_{1}+(a-b) \theta_{2}\right)}+e^{-\frac{2 \pi i}{3}\left((a-b) \theta_{1}+(2 a+b) \theta_{2}\right)} \\
& +e^{-\frac{2 \pi i}{3}\left((a+2 b) \theta_{1}+(-a+b) \theta_{2}\right)}+e^{-\frac{2 \pi i}{3}\left((a+2 b) \theta_{1}+(2 a+b) \theta_{2}\right)} .
\end{aligned}
$$

Note that the orbit function $\phi_{(a \text { a }}(x)$ is real valued for all $a \in \mathbb{Z}^{\geq 0}$ and

$$
\begin{aligned}
\phi_{(a a)}(x) & =2\left\{\cos 2 \pi a\left(\varphi_{1}+\varphi_{2}\right)+\cos 2 \pi a\left(2 \varphi_{2}-\varphi_{1}\right)+\cos 2 \pi a\left(2 \varphi_{1}-\varphi_{2}\right)\right\} \\
& =2\left\{\cos 2 \pi a\left(\theta_{1}+\theta_{2}\right)+\cos 2 \pi a \theta_{1}+\cos 2 \pi a \theta_{2}\right\} .
\end{aligned}
$$

Similarly one finds $\phi_{(a)}(x)$ and $\left.\phi_{(0} b\right)(x)$ :

$$
\begin{aligned}
& \phi_{(a 0)}(x)=e^{\frac{2 \pi i}{3} a\left(2 \theta_{1}+\theta_{2}\right)}+e^{\frac{2 \pi i}{3} a\left(-\theta_{1}+\theta_{2}\right)}+e^{\frac{2 \pi i}{3} a\left(-\theta_{1}-2 \theta_{2}\right)}, \\
& \phi_{(0 b)}(x)=e^{\frac{2 \pi i}{3} b\left(\theta_{1}+2 \theta_{2}\right)}+e^{\frac{2 \pi i}{3} b\left(\theta_{1}-\theta_{2}\right)}+e^{\frac{2 \pi i}{3} b\left(-2 \theta_{1}-\theta_{2}\right)} .
\end{aligned}
$$

Note that the pairs $\phi_{(a b)}(x)+\phi_{(b a)}(x)$ are always real functions.

\subsection{Orbit functions of $C_{2}$ and $G_{2}$}

Let $\lambda=a \omega_{1}+b \omega_{2}=(a b)$ and use the matrices $S$ from (2.6) which are of the form

$$
S\left(C_{2}\right)=\frac{1}{2}\left(\begin{array}{ll}
1 & 1 \\
1 & 2
\end{array}\right), \quad S\left(G_{2}\right)=\frac{1}{3}\left(\begin{array}{ll}
6 & 3 \\
3 & 2
\end{array}\right) .
$$

Then if $x=\left(\theta_{1} \omega_{1}+\theta_{2} \omega_{2}\right)$ for $C_{2}$ and $x=\frac{1}{2}\left(\theta_{1} \omega_{1}+\theta_{2} \omega_{2}\right)$ for $G_{2}$ we find that the orbit functions for $C_{2}$ and $G_{2}$ are of the form

$$
\begin{aligned}
C_{2}: \quad \phi_{(a b)}(x)= & 2 \cos \pi\left((a+b) \theta_{1}+(a+2 b) \theta_{2}\right)+2 \cos \pi\left(b \theta_{1}+(a+2 b) \theta_{2}\right) \\
& +2 \cos \pi\left((a+b) \theta_{1}+a \theta_{2}\right)+2 \cos \pi\left(b \theta_{1}-a \theta_{2}\right), \\
\phi_{(a b)}(x)= & 2 \cos \pi a\left(\theta_{1}+\theta_{2}\right)+2 \cos \pi a \theta_{2}, \\
\phi_{(0 \quad b)}(x)= & 2 \cos \pi b\left(\theta_{1}+2 \theta_{2}\right)+2 \cos \pi b \theta_{1}, \\
G_{2}: \quad \phi_{(a b)}(x)= & \left.2 \cos \pi(2 a+b) \theta_{1}+\left(a+\frac{2}{3} b\right) \theta_{2}\right)+2 \cos \pi\left((a+b) \theta_{1}+\left(a+\frac{2}{3} b\right) \theta_{2}\right) \\
& +2 \cos \pi\left((2 a+b) \theta_{1}+\left(a+\frac{1}{3} b\right) \theta_{2}\right)+2 \cos \pi\left((a+b) \theta_{1}+\frac{1}{3} b \theta_{2}\right) \\
& +2 \cos \pi\left(a \theta_{1}+\left(a+\frac{1}{3} b\right) \theta_{2}\right)+2 \cos \pi\left(a \theta_{1}-\frac{1}{3} b \theta_{2}\right), \\
\phi_{(a b)}(x)= & 2 \cos \pi a\left(2 \theta_{1}+\theta_{2}\right)+2 \cos \pi a\left(\theta_{1}+\theta_{2}\right)+2 \cos \pi a \theta_{1}, \\
\phi_{(0 \quad b)}(x)= & 2 \cos \pi b\left(\theta_{1}+\frac{2}{3} \theta_{2}\right)+2 \cos \pi b\left(\theta_{1}+\frac{1}{3} \theta_{2}\right)+2 \cos \pi \frac{1}{3} b \theta_{2} .
\end{aligned}
$$

As we see, orbit functions for $C_{2}$ and $G_{2}$ are real. 


\subsection{Orbit functions of $A_{n}$}

It is difficult to write down an explicit form for the orbit functions of $A_{n}, B_{n}, C_{n}$ and $D_{n}$ in coordinates with respect to the $\omega$ - and $\alpha$-bases. Thus, in these particular cases we use the orthogonal coordinate systems described in Section 3.

Let $\lambda=\left(m_{1}, m_{2}, \ldots, m_{n+1}\right)$ be an element of $P_{+}\left(A_{n}\right)$ represented in the orthogonal coordinates described in Subsection 3.1. Then $m_{1} \geq m_{2} \geq \cdots \geq m_{n+1}$. The Weyl group in this case coincides with the symmetric group $S_{n+1}$. We denote by $S_{\lambda} \equiv W_{\lambda}$ the subgroup of $W\left(A_{n}\right) \equiv S_{n+1}$ consisting of elements $w \in S_{n+1}$ such that $w \lambda=\lambda$. Then the orbit $O(\lambda)$ consists of points $w \lambda, w \in W / W_{\lambda} \equiv S_{n+1} / S_{\lambda}$. If we represent the points $x \in E_{n+1}$ in the orthogonal coordinate system as well, $x=\left(x_{1}, x_{2}, \ldots, x_{n+1}\right)$, and if we use the formula (6.1) we find that

$$
\begin{aligned}
\phi_{\lambda}(x) & =\sum_{w \in S_{n+1} / S_{\lambda}} e^{2 \pi \mathrm{i}\left\langle w\left(m_{1}, \ldots, m_{n+1}\right),\left(x_{1}, \ldots, x_{n+1}\right)\right\rangle} \\
& =\sum_{w \in S_{n+1} / S_{\lambda}} e^{2 \pi \mathrm{i}\left((w \lambda)_{1} x_{1}+\cdots+(w \lambda)_{n+1} x_{n+1}\right)},
\end{aligned}
$$

where $\left((w \lambda)_{1},(w \lambda)_{2}, \ldots,(w \lambda)_{n+1}\right)$ are orthogonal coordinates of $w \lambda$ if $\lambda=\left(m_{1}, m_{2}, \ldots, m_{n+1}\right)$.

Note that $-\left(m_{n+1}, m_{n}, \ldots, m_{1}\right) \in P_{+}$if $\left(m_{1}, m_{2}, \ldots m_{n+1}\right) \in P_{+}$. In the Weyl group $W\left(A_{n}\right)$ there exists an element $w_{0}$ such that

$$
w_{0}\left(m_{1}, m_{2}, \ldots, m_{n+1}\right)=\left(m_{n+1}, m_{n}, \ldots, m_{1}\right) .
$$

It follows from here that in the expressions for $\phi_{\left(m_{1}, m_{2}, \ldots, m_{n+1}\right)}(x)$ and $\phi_{-\left(m_{n+1}, m_{n}, \ldots, m_{1}\right)}(x)$ there are summands

$$
e^{2 \pi \mathrm{i}\left\langle w_{0} \lambda, x\right\rangle}=e^{2 \pi \mathrm{i}\left(m_{n+1} x_{1}+\cdots+m_{1} x_{n+1}\right)} \quad \text { and } \quad e^{-2 \pi \mathrm{i}\left(m_{n+1} x_{1}+\cdots+m_{1} x_{n+1}\right)},
$$

respectively, which are complex conjugate to each other. Similarly, in the expressions (6.10) for $\phi_{\left(m_{1}, m_{2}, \ldots, m_{n+1}\right)}(x)$ and for $\phi_{-\left(m_{n+1}, m_{n}, \ldots, m_{1}\right)}(x)$ all other summands are pairwise complex conjugate. Therefore,

$$
\phi_{\left(m_{1}, m_{2}, \ldots, m_{n+1}\right)}(x)=\overline{\phi_{-\left(m_{n+1}, m_{n}, \ldots, m_{1}\right)}(x)} .
$$

If we use for $\lambda$ the coordinates $\lambda_{i}=\left\langle\lambda, \alpha_{i}^{\vee}\right\rangle$ in the $\omega$-basis instead of the orthogonal coordinates $m_{j}$, then this equation can be written as

$$
\phi_{\left(\lambda_{1}, \ldots, \lambda_{n}\right)}(x)=\overline{\phi_{\left(\lambda_{n}, \ldots, \lambda_{1}\right)}(x)} .
$$

According to (6.11), if

$$
\left(m_{1}, m_{2}, \ldots, m_{n+1}\right)=-\left(m_{n+1}, m_{n}, \ldots, m_{1}\right)
$$

(that is, the element $\lambda$ has in the $\omega$-basis the coordinates $\left(\lambda_{1}, \lambda_{2}, \ldots, \lambda_{2}, \lambda_{1}\right)$, then the orbit function $\phi_{\lambda}$ is real. Here, the orbit functions can be represented as sums of cosines of the corresponding angles (as in Subsection 6.2).

\subsection{Orbit functions of $B_{n}$}

Let $\lambda=\left(m_{1}, m_{2}, \ldots, m_{n}\right)$ be an element of $P_{+}\left(B_{n}\right)$ in the orthogonal coordinates described in Subsection 3.2. Then $m_{1} \geq m_{2} \geq \cdots \geq m_{n} \geq 0$. The Weyl group $W\left(B_{n}\right)$ consists of permutations of the orthogonal coordinates with sign alternations of some of them. We denote by $S_{\lambda}$ the subgroup of the permutation group $S_{n}$ consisting of elements $w$ such that $w \lambda=\lambda$. If 
we represent the points $x \in E_{n}$ in the orthogonal coordinate system, $x=\left(x_{1}, x_{2}, \ldots, x_{n}\right)$, and if we use formula (6.1) we find that

$$
\begin{aligned}
\phi_{\lambda}(x) & =\sum_{\varepsilon_{i}= \pm 1} \sum_{w \in S_{n} / S_{\lambda}} e^{2 \pi \mathrm{i}\left\langle w\left(\varepsilon_{1} m_{1}, \ldots, \varepsilon_{n} m_{n}\right),\left(x_{1}, \ldots, x_{n}\right)\right\rangle} \\
& =\sum_{\varepsilon_{i}= \pm 1} \sum_{w \in S_{n} / S_{\lambda}} e^{2 \pi \mathrm{i}\left((w(\varepsilon \lambda))_{1} x_{1}+\cdots+(w(\varepsilon \lambda))_{n} x_{n}\right)},
\end{aligned}
$$

where $\left((w(\varepsilon \lambda))_{1}, \ldots,(w(\varepsilon \lambda))_{n}\right)$ are the coordinates of $w(\varepsilon \lambda)$ if $\varepsilon \lambda=\left(\varepsilon_{1} m_{1}, \ldots, \varepsilon_{n} m_{n}\right)$. In $(6.13)$ the summation is over those $\varepsilon_{i}= \pm 1$ for which $m_{i} \neq-m_{i}$.

Since in $W\left(B_{n}\right)$ there exists an element that changes signs of all orthogonal coordinates, for each summand $e^{2 \pi \mathrm{i}\left((w(\varepsilon \lambda))_{1} x_{1}+\cdots+(w(\varepsilon \lambda))_{n} x_{n}\right)}$ in the expressions (6.13) for the orbit function $\phi_{\left(m_{1}, m_{2}, \ldots, m_{n}\right)}(x)$ there exists exactly one summand that is the complex conjugate of it, that is, the summand $e^{-2 \pi \mathrm{i}\left(\left((w(\varepsilon \lambda))_{1} x_{1}+\cdots+(w(\varepsilon \lambda))_{n} x_{n}\right)\right.}$. This means that all orbit functions of $B_{n}$ are real. Each orbit function of $B_{n}$ can be represented as a sum of cosines of the corresponding angles.

\subsection{Orbit functions of $C_{n}$}

Let $\lambda=\left(m_{1}, m_{2}, \ldots, m_{n}\right)$ be an element of $P_{+}\left(C_{n}\right)$ in the orthogonal coordinates described in Subsection 3.3. Then $m_{1} \geq m_{2} \geq \cdots \geq m_{n} \geq 0$. The Weyl group $W\left(C_{n}\right)$ consists of permutations of the coordinates with sign alternations of some of them. We denote by $S_{\lambda}$ the subgroup of $S_{n}$ consisting of elements $w$ such that $w \lambda=\lambda$. If we represent points $x \in E_{n}$ in the orthogonal coordinate system, $x=\left(x_{1}, x_{2}, \ldots, x_{n}\right)$, we find that

$$
\begin{aligned}
\phi_{\lambda}(x) & =\sum_{\varepsilon_{i}= \pm 1} \sum_{w \in S_{n} / S_{\lambda}} e^{2 \pi \mathrm{i}\left\langle w\left(\varepsilon_{1} m_{1}, \ldots, \varepsilon_{n} m_{n}\right),\left(x_{1}, \ldots, x_{n}\right)\right\rangle} \\
& =\sum_{\varepsilon_{i}= \pm 1} \sum_{w \in S_{n} / S_{\lambda}} e^{2 \pi \mathrm{i}\left((w(\varepsilon \lambda))_{1} x_{1}+\cdots+(w(\varepsilon \lambda))_{n} x_{n}\right)},
\end{aligned}
$$

where, as above, $\left((w(\varepsilon \lambda))_{1}, \ldots,(w(\varepsilon \lambda))_{n}\right)$ are the coordinates of the points $w(\varepsilon \lambda)$ if $\varepsilon \lambda=$ $\left(\varepsilon_{1} m_{1}, \ldots, \varepsilon_{n} m_{n}\right)$. In (6.14) the summation is over those $\varepsilon_{i}= \pm 1$ for which $m_{i} \neq-m_{i}$.

In the expressions $(6.14)$ for $\phi_{\left(m_{1}, m_{2}, \ldots, m_{n}\right)}(x)$, for each summand $e^{2 \pi \mathrm{i}\left((w(\varepsilon \lambda))_{1} x_{1}+\cdots+(w(\varepsilon \lambda))_{n} x_{n}\right)}$ there exists exactly one summand complex conjugate to it, that is, $e^{-2 \pi \mathrm{i}\left((w(\varepsilon \lambda))_{1} x_{1}+\cdots+(w(\varepsilon \lambda))_{n} x_{n}\right)}$. Therefore, all orbit functions of $C_{n}$ are real. Each orbit function of $C_{n}$ can be represented as a sum of cosines of the corresponding angles.

\subsection{Orbit functions of $D_{n}$}

Let $\lambda=\left(m_{1}, m_{2}, \ldots, m_{n}\right)$ be an element of $P_{+}\left(D_{n}\right)$ in the orthogonal coordinates described in Subsection 3.4. Then $m_{1} \geq m_{2} \geq \cdots \geq m_{n-1} \geq\left|m_{n}\right|$. The Weyl group $W\left(D_{n}\right)$ consists of permutations of the coordinates with sign alternations for an even number of them. Let $S_{\lambda}$ be the subgroup of the permutation group $S_{n}$ consisting of elements $w$ such that $w \lambda=\lambda$. Representing points $x \in E_{n}$ also in the orthogonal coordinate system, $x=\left(x_{1}, x_{2}, \ldots, x_{n}\right)$, and using formula (6.1) we find that

$$
\begin{aligned}
\phi_{\lambda}(x) & =\sum_{\varepsilon_{i}= \pm 1}^{\prime} \sum_{w \in S_{n} / S_{\lambda}} e^{2 \pi \mathrm{i}\left\langle w\left(\varepsilon_{1} m_{1}, \ldots, \varepsilon_{n} m_{n}\right),\left(x_{1}, \ldots, x_{n}\right)\right\rangle} \\
& =\sum_{\varepsilon_{i}= \pm 1}^{\prime} \sum_{w \in S_{n} / S_{\lambda}} e^{2 \pi \mathrm{i}\left((w(\varepsilon \lambda))_{1} x_{1}+\cdots+(w(\varepsilon \lambda))_{n} x_{n}\right)},
\end{aligned}
$$


where $\left((w(\varepsilon \lambda))_{1}, \ldots,(w(\varepsilon \lambda))_{n}\right)$ are the coordinates of the points $w(\varepsilon \lambda)$ and the prime at the sum sign means that the summation is over values of $\varepsilon_{i}$ with an even number of minus signs if $m_{n} \neq 0$ and, if $m_{n}=0$, over all the $\varepsilon_{i}= \pm 1$ for which $m_{i} \neq-m_{i}$.

Note that in the expressions (6.15) for the orbit function $\phi_{\left(m_{1}, m_{2}, \ldots, m_{n}\right)}(x)$ of $D_{2 k}$ for each summand $e^{2 \pi \mathrm{i}\left((w(\varepsilon \lambda))_{1} x_{1}+\cdots+(w(\varepsilon \lambda))_{n} x_{n}\right)}$ there exists exactly one summand complex conjugate to it. This means that all orbit functions of $D_{2 k}$ are real. Each orbit function of $D_{2 k}$ can be represented as a sum of cosines of the corresponding angles.

It also follows from (6.15) that for $D_{2 k+1}$ an orbit function $\phi_{\left(m_{1}, m_{2}, \ldots, m_{n}\right)}(x)$ is real if and only if the condition $m_{2 k+1}=0$ is fulfilled. The orbit functions $\phi_{\left(m_{1}, \ldots, m_{2 k}, m_{2 k+1}\right)}(x)$ and $\phi_{\left(m_{1}, \ldots, m_{2 k},-m_{2 k+1}\right)}(x)$ of $D_{2 k+1}$ are complex conjugates. If $m_{2 k+1}=0$, then the corresponding orbit function $\phi_{\left(m_{1}, m_{2}, \ldots, m_{n}\right)}(x)$ of $D_{2 k+1}$ can be represented as a sum of cosines of the appropriate angles.

\section{$7 \quad$ Properties of orbit functions}

\subsection{Invariance with respect to affine Weyl group}

Since the scalar product $\langle\cdot, \cdot\rangle$ in $E_{n}$ is invariant with respect to the Weyl group $W$, that is,

$$
\langle w x, w y\rangle=\langle x, y\rangle, \quad w \in W, \quad x, y \in E_{n},
$$

orbit functions $\phi_{\lambda}$ are invariant with respect to $W$ :

$$
\phi_{\lambda}(w x)=\phi_{\lambda}(x), \quad w \in W .
$$

Indeed,

$$
\phi_{\lambda}(w x)=\sum_{\mu \in O(\lambda)} e^{2 \pi \mathrm{i}\langle\mu, w x\rangle}=\sum_{\mu \in O(\lambda)} e^{2 \pi \mathrm{i}\left\langle w^{-1} \mu, x\right\rangle}=\sum_{\mu \in O(\lambda)} e^{2 \pi \mathrm{i}\langle\mu, x\rangle}=\phi_{\lambda}(x)
$$

since $w O(\lambda)=O(\lambda)$ for each $w \in W$.

Let us show that $\phi_{\lambda}$ with $\lambda \in P$ are invariant with respect to the affine Weyl group $W^{\text {aff }}$. Since $W^{\text {aff }}$ is generated by $r_{0}, r_{\alpha_{1}}, \ldots, r_{\alpha_{n}}$ (see Subsection 5.1), it is enough to show invariance of $\phi_{\lambda}$ with respect to $r_{0}: \phi_{\lambda}\left(r_{0} x\right)=\phi_{\lambda}(x)$. Due to (5.2), for $\mu \in P$ we have

$$
\left\langle\mu, r_{0} x\right\rangle=\left\langle\mu, \xi^{\vee}+r_{\xi} x\right\rangle=\frac{2\langle\mu, \xi\rangle}{\langle\xi, \xi\rangle}+\left\langle\mu, r_{\xi} x\right\rangle=\text { integer }+\left\langle r_{\xi} \mu, x\right\rangle
$$

since $r_{\xi}^{2}=1$. Hence,

$$
\phi_{\lambda}\left(r_{0} x\right)=\sum_{\mu \in O(\lambda)} e^{2 \pi \mathrm{i}\left\langle\mu, r_{0} x\right\rangle}=\sum_{\mu \in O(\lambda)} e^{2 \pi \mathrm{i}\left\langle r_{\xi} \mu, x\right\rangle}=\sum_{\mu \in O(\lambda)} e^{2 \pi \mathrm{i}\langle\mu, x\rangle}=\phi_{\lambda}(x)
$$

since $r_{\xi} O(\lambda)=O(\lambda)$.

Note that if $\lambda \notin P_{+}$, then $\phi_{\lambda}$ is not invariant with respect to $W^{\text {aff }}$. It is invariant only under $W$.

Due to the invariance of orbit functions $\phi_{\lambda}, \lambda \in P_{+}$, with respect to $W^{\text {aff }}$, it is enough to consider them only on the fundamental domain $F \equiv F\left(W^{\text {aff }}\right)$ of $W^{\text {aff }}$. Values of $\phi_{\lambda}$ on other points of $E_{n}$ are determined by using the action of $W^{\text {aff }}$ on $F$ or taking a limit. In particular, orbit functions $\phi_{\lambda}, \lambda \in P_{+}$, are invariant under the reflection of $F$ with respect to any $(n-1)$ dimensional wall of the fundamental domain $F$. 


\subsection{Continuity}

An orbit function $\phi_{\lambda}$ is a finite sum of exponential functions. Therefore it is continuous and has continuous derivatives of all orders in $E_{n}$.

The normal derivative of $\phi_{\lambda}(x)$ to the boundary of $F$ equals zero. It follows from the continuity of $\phi_{\lambda}(x)$ and of its derivatives, together with its invariance.

\subsection{Realness and complex conjugation}

The results formulated below and concerning orbit functions of the Coxeter-Dynkin diagrams $A_{n}, B_{n}, C_{n}$ and $D_{n}$ were proved in the previous section. Other cases can be proved, for example, by means of the representation theory of compact Lie groups; these proofs are omitted.

Orbit functions of the following Coxeter-Dynkin diagrams are real:

$$
A_{1}, B_{n}, C_{n}, D_{2 k}, E_{7}, E_{8}, F_{4}, G_{2} \text {. }
$$

The orbit functions $\phi_{\lambda}$ of the remaining Coxeter-Dynkin diagrams are real, provided the dominant weights $\lambda$ are invariant with respect to the symmetry transformation of the diagram. For that the coordinates $\lambda_{1}, \lambda_{2}, \ldots, \lambda_{n}, \lambda_{i}=\left\langle\lambda, \alpha_{i}^{\vee}\right\rangle$, of $\lambda$ have to be of the form

$$
\begin{aligned}
& \left(\begin{array}{llllll}
\lambda_{1} & \lambda_{2} & \cdots & \lambda_{2} & \lambda_{1}
\end{array}\right) \quad \text { for } A_{n} \quad(n \geq 2) \text {, } \\
& \left(\begin{array}{lllllll}
\lambda_{1} & \lambda_{2} & \cdots & \lambda_{2 k-1} & \lambda_{2 k} & \lambda_{2 k}
\end{array}\right) \text { for } D_{2 k+1} \quad(k \geq 2) \text {, } \\
& \left(\begin{array}{llllll}
\lambda_{1} & \lambda_{2} & \lambda_{3} & \lambda_{2} & \lambda_{1} & \lambda_{6}
\end{array}\right) \quad \text { for } E_{6} \text {. }
\end{aligned}
$$

Orbit functions corresponding to the following pairs of dominant weights are complex conjugate:

$$
\begin{aligned}
& \left(\begin{array}{llll}
\lambda_{1} & \lambda_{2} & \cdots & \lambda_{n}
\end{array}\right) \quad\left(\lambda_{n} \lambda_{n-1} \cdots \lambda_{1}\right) \quad \text { for } A_{n}, \\
& \left(\begin{array}{lllllllll}
\lambda_{1} & \lambda_{2} & \cdots & \lambda_{2 k-1} & \lambda_{2 k+1} & \lambda_{2 k}
\end{array}\right) \quad\left(\begin{array}{llllll}
\lambda_{1} & \lambda_{2} & \cdots & \lambda_{2 k-1} & \lambda_{2 k} & \lambda_{2 k+1}
\end{array}\right) \text { for } D_{2 k+1} \text {, } \\
& \left.\begin{array}{llllll}
\lambda_{1} & \lambda_{2} & \lambda_{3} & \lambda_{4} & \lambda_{5} & \lambda_{6}
\end{array}\right) \quad\left(\begin{array}{llllll}
\lambda_{5} & \lambda_{4} & \lambda_{3} & \lambda_{2} & \lambda_{1} & \lambda_{6}
\end{array}\right) \quad \text { for } E_{6} \text {. }
\end{aligned}
$$

\subsection{Scaling symmetry}

Let $O(\lambda)$ be an orbit of $\lambda \in E_{n}$. Since $w(c \lambda)=c w(\lambda)$ for any $c \in \mathbb{R}$ and for any $w \in W$, then $O(c \lambda)$ is the orbit consisting of the points $c w \lambda, w \in W$. Let $\phi_{\lambda}=\sum_{\mu \in O(\lambda)} e^{2 \pi \mathrm{i} \mu}$ be the orbit function for $\lambda \in E_{n}$. Then

$$
\phi_{c \lambda}(x)=\sum_{c \mu \in O(c \lambda)} e^{2 \pi \mathrm{i}\langle c \mu, x\rangle}=\sum_{\mu \in O(\lambda)} e^{2 \pi \mathrm{i}\langle\mu, c x\rangle}=\phi_{\lambda}(c x) .
$$

The equality $\phi_{c \lambda}(x)=\phi_{\lambda}(c x)$ expresses the scaling symmetry of orbit functions.

If we deal only with orbit functions $\phi_{\lambda}$, corresponding to $\lambda \in P_{+}$, then the scaling symmetry $\phi_{c \lambda}(x)=\phi_{\lambda}(c x)$ holds for values $c \in \mathbb{R} \backslash\{0\}$ such that $c \lambda \in P_{+}$.

\subsection{Duality}

The modified orbit functions $\hat{\phi}_{\lambda}(x)$ (see formula (6.2)) can be represented in the form

$$
\hat{\phi}_{\lambda}(x)=\sum_{w \in W} e^{2 \pi \mathrm{i}\langle w \lambda, x\rangle}
$$


Due to the invariance of the scalar product $\langle\cdot, \cdot\rangle$ with respect to the Weyl group $W,\langle w \mu, w y\rangle=$ $\langle\mu, y\rangle$, we have

$$
\hat{\phi}_{\lambda}(x)=\sum_{w \in W} e^{2 \pi \mathrm{i}\left\langle\lambda, w^{-1} x\right\rangle}=\sum_{w \in W} e^{2 \pi \mathrm{i}\langle\lambda, w x\rangle}=\hat{\phi}_{x}(\lambda) .
$$

The relation $\hat{\phi}_{\lambda}(x)=\hat{\phi}_{x}(\lambda)$ expresses the duality of orbit functions.

\subsection{Orthogonality}

Orbit functions $\phi_{\lambda}, \lambda \in P_{+}$, are orthogonal on $\bar{F}$ with respect to the Euclidean measure:

$$
|\bar{F}|^{-1} \int_{\bar{F}} \phi_{\lambda}(x) \overline{\phi_{\lambda^{\prime}}(x)} d x=|O(\lambda)| \delta_{\lambda \lambda^{\prime}}
$$

where the overbar denotes the complex conjugate. This relation directly follows from the orthogonality of the exponential functions (entering into the definition of orbit functions) for different weights $\mu$ and from the fact that a given weight $\nu \in P$ belongs to precisely one orbit function. In (7.4), $|F|$ means an area of the fundamental domain $F$.

It is evident that for modified orbit functions (6.2) the orthogonality relation is of the form

$$
|\bar{F}|^{-1} \int_{\bar{F}} \hat{\phi}_{\lambda}(x) \overline{\hat{\phi}_{\lambda^{\prime}}(x)} d x=\left|W_{\lambda}\right|^{2}|O(\lambda)| \delta_{\lambda \lambda^{\prime}}=\left|W_{\lambda}\right||W| \delta_{\lambda \lambda^{\prime}}
$$

\subsection{Properties of orbit functions of $A_{n}$}

In this subsection we consider properties of orbit functions of $A_{n}$ which follows from properties of symmetric polynomials (see [5]).

We represent orbit functions of $A_{n}$ in the orthogonal coordinate system as in formula (6.10). Let $\lambda=\left(m_{1}, \ldots, m_{n+1}\right)$ and $\lambda+\boldsymbol{m}=\left(m_{1}+m, \ldots, m_{n+1}+m\right)$, where $m$ is a fixed real number. If $x=\left(x_{1}, \ldots, x_{n+1}\right), x_{1}+\cdots+x_{n+1}=0$, and $w \in W$, then we have

$$
e^{2 \pi \mathrm{i}\langle\lambda+\boldsymbol{m}, w x\rangle}=e^{2 \pi \mathrm{i}\langle\lambda, w x\rangle} e^{2 \pi \mathrm{i}\langle 0+\boldsymbol{m}, w x\rangle}=e^{2 \pi \mathrm{i}\langle\lambda, w x\rangle} .
$$

It follows from this equality that

$$
\phi_{\lambda}(x)=\phi_{\lambda+\boldsymbol{m}}(x)
$$

where $\lambda=\left(m_{1}, \ldots, m_{n+1}\right)$ is given in the orthogonal coordinate system. This means that instead of $m_{i}, i=1,2, \ldots, n+1$, determined by formulas of Subsection 3.1, we may assume that $m_{1}, \ldots, m_{n+1}$ are integers such that $m_{1} \geq m_{2} \geq \cdots \geq m_{n+1} \geq 0$. We adopt this assumption in this subsection.

For simplicity we introduce the following notations:

$$
e^{2 \pi \mathrm{i} x_{j}}=y_{j}, \quad j=1,2, \ldots, n+1 .
$$

The element

$$
\lambda=\left(m_{1}, \ldots, m_{n+1}\right) \equiv(1,1, \ldots, 1,0, \ldots, 0)
$$

with $r$ units will be denoted by $\left(1^{r}\right)$ (it is the fundamental weight $\left.\omega_{r}\right)$. Then from formula (6.10) for orbit functions of $A_{n}$ one gets

$$
\phi_{1^{r}}(x)=\sum_{i_{1}<i_{2}<\cdots<i_{r}} y_{i_{1}} y_{i_{2}} \cdots y_{i_{r}} .
$$


It is easy to show using this formula that

$$
\mathrm{F}(x ; t):=\prod_{i=1}^{n+1}\left(1+y_{i} t\right)=\sum_{r=0}^{n+1} \phi_{1^{r}}(x) t^{r},
$$

that is, the function $\mathrm{F}(x ; t) \equiv \mathrm{F}\left(x_{1}, \ldots, x_{n+1} ; t\right)$ is a generating function for $n+1$ orbit functions $\phi_{1^{r}}(x)$.

Now we denote by $\Phi_{r}(x) \equiv \Phi_{r}\left(x_{1}, \ldots, x_{n+1}\right), r=1,2, \ldots$, the functions

$$
\Phi_{r}(x)=\sum_{|\lambda|=r} \phi_{\lambda}(x)
$$

where $|\lambda|=m_{1}+m_{2}+\cdots+m_{n+1}$ if $\lambda=\left(m_{1}, m_{2}, \ldots, m_{n+1}\right)$. Since $\left(1-y_{i} t\right)^{-1}=\sum_{k=0}^{\infty} y_{i}^{k} t^{k}$, then

$$
B(x ; t):=\prod_{i=1}^{n+1}\left(1-y_{i} t\right)^{-1}=\sum_{r=0}^{\infty} \Phi_{r}(x) t^{r},
$$

where $x_{i}$ and $y_{i}$ are related in the same manner as before. Thus, $B(x ; t)$ is a generating function for $\Phi_{r}(x)$. Since $\mathrm{F}(x ;-t) B(x ; t)=1$, after comparing coefficients at fixed $t^{s}$ we obtain the equality

$$
\sum_{r=0}^{s}(-1)^{r} \phi_{1^{r}}(x) \Phi_{r}(x)=0 .
$$

It follows from formula (6.10) that for $\lambda=(r, 0, \ldots, 0) \equiv(r, \mathbf{0}), r=0,1,2, \ldots$, we have

$$
\phi_{(r, \mathbf{0})}(x)=\sum_{i=1}^{n+1} y_{i}^{r}
$$

where, as before, $y_{i}=e^{2 \pi \mathrm{i} x_{i}}$. The relations

$$
P(x ; t):=\sum_{i=1}^{n+1} \frac{y_{i}}{1-y_{i} t}=\sum_{i=1}^{n+1} \sum_{r=1}^{\infty} y_{i}^{r} t^{r-1}=\sum_{r=1}^{\infty} \phi_{(r, \mathbf{0})}(x) t^{r-1}
$$

show that $P(x ; t)$ is a generating function for the orbit functions $\phi_{(r, \mathbf{0})}(x)$.

It is easy to show that

$$
P(x ; t)=B^{\prime}(x ; t) / B(x ; t), \quad P(x ;-t)=\mathrm{F}^{\prime}(x ; t) / \mathrm{F}(x ; t),
$$

where derivatives are taken with respect to variable $t$. Representing these equalities in the form $P(x ; t) B(x ; t)=B^{\prime}(x ; t)$ and $P(x ;-t) \mathrm{F}(x ; t)=\mathrm{F}^{\prime}(x ; t)$, substituting here the corresponding expressions in terms of orbit functions and then comparing coefficients at fixed $t^{s}$, one gets

$$
s \Phi_{s}(x)=\sum_{r=1}^{s} \phi_{(r, \mathbf{0})}(x) \Phi_{s-r}(x), \quad s \phi_{1^{s}}(x)=\sum_{r=1}^{s}(-1)^{r-1} \phi_{(r, \mathbf{0})}(x) \phi_{1^{s-r}}(x) .
$$

Proofs of the equalities

$$
\phi_{(r, \mathbf{0})}=\operatorname{det}\left(\begin{array}{ccccc}
\phi_{1^{1}} & 1 & 0 & \cdots & 0 \\
2 \phi_{1^{2}} & \phi_{1^{1}} & 1 & \cdots & 0 \\
\cdots & \cdots & \ldots & \ldots & \ldots \\
r \phi_{1^{r}} & \phi_{1^{r-1}} & \phi_{1^{r-2}} & \cdots & 1
\end{array}\right),
$$




$$
\phi_{1^{r}}=\frac{1}{r !} \operatorname{det}\left(\begin{array}{ccccc}
\phi_{(1, \mathbf{0})} & 1 & 0 & \cdots & 0 \\
\phi_{(2, \mathbf{0})} & \phi_{(1, \mathbf{0})} & 2 & \cdots & 0 \\
\cdots & \cdots & \cdots & \cdots & \cdots \\
\phi_{(r-1, \mathbf{0})} & \phi_{(r-2, \mathbf{0})} & \phi_{(r-3, \mathbf{0})} & \cdots & r-1 \\
\phi_{(r, \mathbf{0})} & \phi_{(r-1, \mathbf{0})} & \phi_{(r-2, \mathbf{0})} & \cdots & \phi_{(1, \mathbf{0})}
\end{array}\right)
$$

are more complicated and we omit them (the corresponding proofs for symmetric polynomials see in $[5])$.

\subsection{Operations with orbit functions}

According to formula (7.4), orbit functions are orthogonal on the fundamental domain. As we shall see in next section, they form an orthogonal basis in the Hilbert space of squared integrable functions on $F$. Therefore, we may expand functions on $F$ as sums of orbit functions. In particular, products of orbit functions can be uniquely decomposed in orbit functions, corresponding to the same Weyl group. For such decomposition we need to use the results on decomposition of products of orbits (see Section 4).

Let elements $\lambda$ and $\lambda^{\prime}$ be dominant, and let the product of the orbits $O(\lambda)$ and $O\left(\lambda^{\prime}\right)$ decompose as

$$
O(\lambda) \otimes O\left(\lambda^{\prime}\right)=\bigcup_{\nu} O(\nu)
$$

Then for the product of the corresponding orbit functions we have

$$
\phi_{\lambda}(x) \phi_{\lambda^{\prime}}(x)=\sum_{\nu} \phi_{\nu}(x)
$$

where the summation is the same as in (7.6). Indeed, since according to the definition (6.1) we have $\phi_{\lambda}(x)=\sum_{\mu \in O(\lambda)} e^{2 \pi \mathrm{i}\langle\mu, x\rangle}$, then due to $(7.6)$

$$
\begin{aligned}
\phi_{\lambda}(x) \phi_{\lambda^{\prime}}(x) & =\sum_{\mu \in O(\lambda)} e^{2 \pi \mathrm{i}\langle\mu, x\rangle} \sum_{\mu^{\prime} \in O\left(\lambda^{\prime}\right)} e^{2 \pi \mathrm{i}\left\langle\mu^{\prime}, x\right\rangle} \\
& =\sum_{\mu \in O(\lambda)} \sum_{\mu^{\prime} \in O\left(\lambda^{\prime}\right)} e^{2 \pi \mathrm{i}\left\langle\mu+\mu^{\prime}, x\right\rangle}=\sum_{\nu} \sum_{\sigma_{\nu} \in O(\nu)} e^{2 \pi \mathrm{i}\left\langle\sigma_{\nu}, x\right\rangle}=\sum_{\nu} \phi_{\nu}(x) .
\end{aligned}
$$

Each of Propositions 1-4 of Subsection 4.1 can be formulated in terms of the corresponding orbit functions. For example, Proposition 1 says that the product $\phi_{\lambda}(x) \phi_{\mu}(x)$ is decomposed into orbits of the form $\phi_{|w \lambda+\mu|}(x), w \in W / W_{\lambda}$. Proposition 2 states that if all elements $w \lambda+\mu$, $w \in W / W_{\lambda}$, are strictly dominant, then

$$
\phi_{\lambda}(x) \phi_{\mu}(x)=\sum_{w \in W / W_{\lambda}} \phi_{w \lambda+\mu}(x) .
$$

According to Proposition 3, if all elements $w \lambda+\mu, w \in W / W_{\lambda}$, are dominant, then

$$
\phi_{\lambda}(x) \phi_{\mu}(x)=\sum_{w \in W / W_{\lambda}} n_{w \lambda+\mu} \phi_{w \lambda+\mu}(x)
$$

where $n_{w \lambda+\mu}=\left|W_{w \lambda+\mu}\right|$. Proposition 4 can also be easily formulated in terms of orbit functions.

In the cases $A_{2}, B_{2}$ and $G_{2}$, products $\phi_{\lambda}(x) \phi_{\mu}(x)$ are decomposed as sums of orbit functions by using results of Subsection 4.2. For example, for orbit functions of $G_{2}$ we have

$$
\phi_{(a 0)}(x) \phi_{(b 0)}(x)=\phi_{(a+b 0)}(x)+\phi_{(b-a 3 a)}(x)+\phi_{(2 a-b 3 b-3 a)}(x)+\phi_{(b-a 0)}(x)
$$


if $a<b<2 a$ and

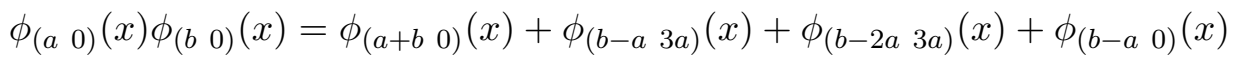

if $b>2 a$.

Decomposition of $W$-orbits into $W^{\prime}$-orbits (see Subsection 4.3) can also be applied in the decomposition of orbit functions. Let $R$ be a root system with a Weyl group $W$, and let $R^{\prime}$ be another root system which is a subsystem of $R$. Let $R$ span a linear space $E_{n}$ and $R^{\prime}$ span its subspace $E_{m}\left(E_{m}\right.$ can coincide with $\left.E_{n}\right)$. If $\phi_{\lambda}(x)$ is a $W$-orbit function and $E_{m} \neq E_{n}$, then we may restrict this function onto the subspace $E_{m}$. Then the $W$-orbit function $\phi_{\lambda}(x)$, considered as a function on $E_{m}$, can be expanded into a sum of $W^{\prime}$-orbit functions. Namely, if $O_{W}(\lambda)=\cup_{j=1}^{s} O_{W^{\prime}}\left(\mu_{j}\right)$, then

$$
\phi_{\lambda}^{(W)}(x)=\sum_{i=1}^{s} \phi_{\mu_{j}}^{\left(W^{\prime}\right)}(x), \quad x \in E_{m} .
$$

In particular, all results of Subsections 4.4-4.9 can be formulated in terms of the corresponding orbit functions. For example, for $W\left(A_{n}\right)$ - and $W\left(A_{n-1}\right)$-orbit functions we have

$$
\phi_{\left(m_{1}, \ldots, m_{n+1}\right)}^{\left(W\left(A_{n}\right)\right)}(x)=\sum_{i=1}^{n+1} \phi_{\left(m_{1}, \ldots, m_{i-1} \hat{m}_{i}, m_{i+1}, \ldots, m_{n+1}\right)}^{\left(W\left(A_{n-1}\right)\right)}(x), \quad x \in E_{n},
$$

where the notations of Subsection 4.4 is used.

The results of Subsection 4.5 give the following decomposition of orbit functions of $A_{n-1}$ as a sum of orbit functions of $A_{p-1} \times A_{q-1}$ with $p+q=n$ :

$$
\phi_{\left(m_{1}, \ldots, m_{n}\right)}^{\left(W\left(A_{n-1}\right)\right)}(x)=\sum_{\left(m_{i_{1}}, \ldots, m_{i_{p}}\right) \in \Sigma} \phi_{\left(m_{i_{1}}, \ldots, m_{i_{p}}\right)}^{\left(W\left(A_{p-1}\right)\right)}(y) \phi_{\left(m_{j_{1}}, \ldots, m_{j_{q}}\right)}^{\left(W\left(A_{q-1}\right)\right)}(z),
$$

where $\Sigma$ is defined in Subsection $4.5,\left(m_{j_{1}}, \ldots, m_{j_{q}}\right)$ is a supplement of the set $\left(m_{i_{1}}, \ldots, m_{i_{p}}\right)$ in $\left(m_{1}, m_{2}, \ldots, m_{n}\right)$, and $y \in E_{p}, z \in E_{q}, x=(y, z)$.

If $E_{m}=E_{n}$, then each $W$-orbit function, as a function on $E_{n}$, can be represented as a sum of $W^{\prime}$-orbit functions. Namely, if $O_{W}(\lambda)=\cup_{i=1}^{s} O_{W^{\prime}}\left(w_{i} \lambda\right)$ (see Subsection 4.3), then

$$
\phi_{\lambda}^{(W)}(x)=\sum_{i=1}^{s} \phi_{w_{i} \lambda}^{\left(W^{\prime}\right)}(x), \quad x \in E_{n} .
$$

\subsection{Congruence classes of orbit functions}

The set of all $W$-orbit functions $\phi_{\lambda}(x)$ can be sorted out into meaningful subsets according to a number of criteria which may be imposed on either of the two variables $\lambda$ or $x$. We point out here such example related to the notion of congruence classes of weights $\lambda$.

Congruence classes of $\lambda \in P$ were introduced in [32]. Each $\lambda \in P$ belongs to a single congruence class. That is, to each $\lambda$ one associates a congruence number $c(\lambda)$ which is a nonnegative integer.

For example, the congruence number $c(\lambda)$ of a point $\lambda=m \omega$ of the weight lattice of $A_{1}$, is given by $c(m \omega)=m \bmod 2$. For the rank 2 cases, we have $\lambda=a \omega_{1}+b \omega_{2}$. Then for $\lambda \in P$ the congruence number $c(\lambda)$ is

$$
c\left(a \omega_{1}+b \omega_{2}\right)= \begin{cases}2 a+b \bmod 3 & \text { for } A_{2}, \\ a \bmod 2 & \text { for } C_{2}, \\ 0 & \text { for } G_{2} .\end{cases}
$$


All the roots of a fixed root system $R$ have congruence number 0 . All weights in a $W$-orbit $O(\lambda), \lambda \in P_{+}$, have the same congruence number. Under multiplication of orbits $O(\lambda)$ and $O(\mu)$ of congruence numbers $c(\lambda)$ and $c(\mu)$, respectively, we obtain a set of weights of the same congruence number $c(\lambda)+c(\mu)$. Therefore, the product $O(\lambda) \otimes O(\mu), \lambda \in P_{+}, \mu \in P_{+}$, is decomposed into orbits with the same congruence number.

If $\lambda \in P_{+}$is of a congruence class $c(\lambda)$, then we say that the orbit function $\phi_{\lambda}(x)$ is of congruence class $c(\lambda)$.

We associate with an orbit function $\phi_{\lambda}, \lambda \in P_{+}$, the congruence number $c(\lambda)$. Then, as in the case of product of orbits, under multiplication of orbit functions their congruence numbers are added up. Moreover, all orbit functions in the decomposition of a product of orbit functions carry the same congruence number, namely $c(\lambda)+c\left(\lambda^{\prime}\right)$.

\section{Orbit function transform}

The exponential functions $e^{2 \pi \mathrm{i}\langle p, x\rangle}, p=\left(p_{1}, p_{2}, \ldots, p_{n}\right), p_{i} \in \mathbb{Z}$, given on $E_{n}$, determine the Fourier series. As we have seen, symmetrization of exponential functions leads to orbit functions. The last functions determine symmetrized Fourier transform, which is also called an orbit function transform, and is a generalization of the decomposition into cos-functions [22]. In order to see this, we first consider a relation of orbit functions to characters of irreducible representations of compact Lie groups.

\subsection{Orbit functions and representation characters}

To each Coxeter-Dynkin diagram there corresponds a connected compact semisimple Lie group $G$. Let us fix a Coxeter-Dynkin diagram and, therefore, a connected compact Lie group $G$. A complex valued function $f(g)$ on $G$ satisfying the condition

$$
f(g)=f\left(h g h^{-1}\right), \quad h \in G,
$$

is called a class function. It is constant on classes of conjugate elements.

For simplicity, we assume that $G$ is realized by matrices such that the set of its diagonal matrices constitutes a Cartan subgroup, which will be denoted by $H$. This subgroup can be identified with the $n$-dimensional torus T, where $n$ is the rank of the group $G$. The subgroup $H$ can be represented as $H=\exp (i \mathfrak{h})$, where $\mathfrak{h}$ is the real form of an appropriate Cartan subalgebra of the complex semisimple Lie algebra, determined by the Coxeter-Dynkin diagram.

It is well-known that each element $g$ of $G$ is conjugate to some element of $H$, that is, class functions are uniquely determined by their values on $H$.

There exists a one-to-one correspondence between irreducible unitary representations of the group $G$ and integral highest weights $\lambda \in P_{+}$, where $P_{+}$is determined by the Coxeter-Dynkin diagram (see, for example, [19] and [33]). The irreducible representation, corresponding to a highest weight $\lambda \in P_{+}$, will be denoted by $T_{\lambda}$. The representation $T_{\lambda}$ and its properties are determined by its character $\chi_{\lambda}(g)$, which is defined as the trace of $T_{\lambda}(g)$ :

$$
\chi_{\lambda}(g)=\operatorname{Tr} T_{\lambda}(g), \quad g \in G .
$$

Since $\operatorname{Tr} T_{\lambda}\left(g^{\prime} g g^{-1}\right)=\operatorname{Tr} T_{\lambda}(g), g^{\prime} \in G$, the character $\chi_{\lambda}$ is a class function, that is, it is uniquely determined by its values on the subgroup $H$.

All the operators $T_{\lambda}(h), h \in H$, are diagonal with respect to an appropriate basis of the representation space (this basis is called a weight basis) and their diagonal matrix elements are of the form $e^{2 \pi \mathrm{i}\langle\mu, x\rangle}$, where $\mu \in P$ is a weight of the representation $T_{\lambda}, x=\left(x_{1}, x_{2}, \ldots, x_{n}\right)$ are coordinates of an element $t$ of the Cartan subalgebra $\mathfrak{h}$ in an appropriate coordinate system 
(they are coordinates on the torus $\mathrm{T}$ ) and $\langle\cdot, \cdot\rangle$ is an appropriate bilinear form, which can be chosen coinciding with the scalar product on $E_{n}$, considered above. Then the character $\chi_{\lambda}(h)$ is a linear combination of the diagonal matrix elements:

$$
\chi_{\lambda}(h)=\sum_{\mu \in D(\lambda)} c_{\lambda}^{\mu} e^{2 \pi i\langle\mu, x\rangle}, \quad h \in H,
$$

where $D(\lambda)$ is the set of all weights of the irreducible representation $T_{\lambda}$ and $c_{\lambda}^{\mu}$ is a multiplicity of the weight $\mu \in D(\lambda)$ in the representation $T_{\lambda}$. It is known from representation theory that the weight system $D(\lambda)$ of $T_{\lambda}$ is invariant with respect to the Weyl group $W$, corresponding to the Coxeter-Dynkin diagram, and $c_{\lambda}^{w \mu}=c_{\lambda}^{\mu}, w \in W$, for each $\mu \in D(\lambda)$. This means that the character $\chi_{\lambda}(h)$ can be represented as

$$
\chi_{\lambda}(h)=\sum_{\mu \in D_{+}(\lambda)} c_{\lambda}^{\mu} \phi_{\mu}(x),
$$

where $D_{+}(\lambda)$ is the set of all dominant weights in $D(\lambda)$ and $\phi_{\mu}(x)$ is the orbit function, corresponding to the weight $\mu \in D_{+}(\lambda)$. Representing $\chi_{\lambda}(h)$ as $\chi_{\lambda}(x)$, where $x=\left(x_{1}, x_{2}, \ldots, x_{n}\right)$ are coordinates, corresponding to the element $t \in \mathfrak{h}$ such that $h=\exp (2 \pi \mathrm{i} t)$, we can make an analytic continuation of both sides of (8.2) to the $n$-dimensional Euclidean space $E_{n}$. Since the right hand side of (8.2) is invariant under transformations from the affine Weyl group $W^{\text {aff }}$, corresponding to the Weyl group $W$, the function $\chi_{\lambda}(x)$ is also invariant under the affine Weyl group $W^{\text {aff }}$. That is, it is enough to define $\chi_{\lambda}(x)$ only on the fundamental domain $F$ of the group $W^{\text {aff }}$. To this fundamental domain $F$ there corresponds a fundamental domain (we denote it by $\tilde{F}$ ) in the subgroup $H$ (and in the torus $\mathrm{T}$ ).

Many properties of orbit functions follow from properties of characters $\chi_{\lambda}$, which we consider known from representation theory.

As one of the reasons, why characters are rarely used in extensive applications, one may bring forward the need to know the multiplicities $c_{\lambda}^{\mu}$ in (8.1). They can be calculated using a laborious recursive algorithm, starting from the highest weight $\lambda$. In many situations it is practical to read off their values from the tables (see [23] and [34]).

\subsection{Orbit function transform on $\bar{F}$}

Let $f(g)$ be a continuous class function on $G$. It defines a continuous function on $H$. We assume that this function on $H$ has continuous partial derivatives of all orders with respect to analytic parameters on $H$. Such function $f$ can be decomposed in characters of irreducible unitary representations of $G$ :

$$
f(h)=\sum_{\lambda \in P_{+}} a_{\lambda} \chi_{\lambda}(h) .
$$

We see from this decomposition that each class function is uniquely determined by its values on the fundamental domain $\tilde{F}$. Moreover, we can state that each continuous function with continuous derivatives on $\tilde{F}$ can be decomposed into series in characters. In particular, we may decompose an orbit function (as a continuous function with continuous derivatives) into series in characters of irreducible representations of $G$ :

$$
\phi_{\mu}(x)=\sum_{\lambda \in P_{+}} a_{\lambda}^{\mu} \chi_{\lambda}(x) .
$$

In real, orbit functions are finite linear combinations of irreducible characters. Indeed, the decomposition (8.2) is the following decomposition

$$
\chi_{\lambda}(x)=\sum_{0 \leq \mu \leq \lambda} c_{\lambda}^{\mu} \phi_{\mu}(x), \quad c_{\lambda}^{\lambda} \neq 0,
$$


where $\mu \leq \lambda$ means that $\lambda-\mu$ belongs to the positive root lattice $Q_{+}$or $\lambda=\mu$, and $\mu \geq 0$ means that $\mu$ is dominant. Let $\mu_{1}, \mu_{2}, \ldots, \mu_{s}$ be the set of all dominant integral weights such that $\mu_{i} \leq \lambda$. Then the characters $\chi_{\mu_{i}}(x), i=1,2, \ldots, s$, are linearly independent. The orbit functions $\phi_{\mu_{i}}(x), i=1,2, \ldots, s$, are also linearly independent since they are pairwise orthogonal. Due to (8.4) the set $\chi_{\mu_{i}}(x), i=1,2, \ldots, s$, is a span of $\phi_{\mu_{i}}(x), i=1,2, \ldots, s$. Besides, the set $\phi_{\mu_{i}}(x), i=1,2, \ldots, s$, and the set $\chi_{\mu_{i}}(x), i=1,2, \ldots, s$, span finite dimensional linear spaces of functions on $F$ of the same dimension. Therefore, these spaces coincide and each $\phi_{\mu_{i}}(x)$ is a linear combination of $\chi_{\mu_{j}}(x), j=1,2, \ldots, s$. Thus, our assertion is proved.

We conclude that each continuous function on $\bar{F}$ with continuous derivatives can be expanded into orbit functions $\phi_{\lambda}, \lambda \in P_{+}$:

$$
f(x)=\sum_{\lambda \in P_{+}} c_{\lambda} \phi_{\lambda}(x)
$$

Due to the orthogonality relation (7.4) for orbit functions, the coefficients $c_{\lambda}$ in this decomposition are determined by the formula

$$
c_{\lambda}=|O(\lambda)|^{-1}|\bar{F}|^{-1} \int_{\bar{F}} f(x) \overline{\phi_{\lambda}(x)} d x
$$

Moreover, the Plancherel formula

$$
\sum_{\lambda \in P_{+}}|O(\lambda)|\left|c_{\lambda}\right|^{2}=|\bar{F}|^{-1} \int_{\bar{F}}|f(x)|^{2} d x
$$

holds. Formula (8.6) is the symmetrized Fourier transform of the function $f(x)$. Formula (8.5) gives an inverse transform. Formulas (8.5) and (8.6) give the orbit function transforms.

For the modified orbit functions (6.2) the relations (8.5)-(8.7) can be written as

$$
\begin{aligned}
& f(x)=\sum_{\lambda \in P_{+}} c_{\lambda}^{\prime} \hat{\phi}_{\lambda}(x), \\
& c_{\lambda}^{\prime}=\left|W_{\lambda}\right|^{-1}|W|^{-1}|\bar{F}|^{-1} \int_{\bar{F}} f(x) \overline{\hat{\phi}_{\lambda}(x)} d x, \\
& \sum_{\lambda \in P_{+}}\left|W_{\lambda}\right||W|\left|c_{\lambda}^{\prime}\right|^{2}=|\bar{F}|^{-1} \int_{\bar{F}}|f(x)|^{2} d x,
\end{aligned}
$$

where $c_{\lambda}^{\prime}=c_{\lambda} /\left|W_{\lambda}\right|$.

Let $\mathcal{L}^{2}(\bar{F})$ denote the Hilbert space of functions on the closure $\bar{F}$ of the fundamental domain $\bar{F}$ with the scalar product

$$
\left\langle f_{1}, f_{2}\right\rangle=|\bar{F}|^{-1} \int_{\bar{F}} f_{1}(x) \overline{f_{2}(x)} d x .
$$

The set of continuous functions on $\bar{F}$ with continuous derivatives is dense in $\mathcal{L}^{2}(\bar{F})$. Therefore, the formulas (8.5)-(8.7) can be continued to functions of $\mathcal{L}^{2}(\bar{F})$. These formulas show that the set of orbit functions $\phi_{\lambda}, \lambda \in P_{+}$, form an orthogonal basis of $\mathcal{L}^{2}(\bar{F})$.

\subsection{Orbit function transform on the dominant Weyl chamber}

The expansion (8.5) of functions on the fundamental domain $F$ is an expansion in the orbit functions $\phi_{\lambda}(x), \lambda \in P_{+}$. Other orbit functions $\phi_{\lambda}(x), \lambda \in E_{n}$, are not invariant with respect to the corresponding affine Weyl group $W^{\text {aff }}$. They are invariant only with respect to the Weyl 
group $W$. A fundamental domain of $W$ coincides with the dominant Weyl chamber $D_{+}$. For this reason, the orbit functions $\phi_{\lambda}(x), \lambda \in E_{n}$, determine another orbit function transform (a transform on $D_{+}$).

Let us start with the usual Fourier transforms on $\mathbb{R}^{n}$ :

$$
\begin{aligned}
\tilde{f}(\lambda) & =\int_{-\infty}^{\infty} f(x) e^{2 \pi \mathrm{i}\langle\lambda, x\rangle} d x, \\
f(x) & =\int_{-\infty}^{\infty} \tilde{f}(\lambda) e^{-2 \pi \mathrm{i}\langle\lambda, x\rangle} d \lambda .
\end{aligned}
$$

Let the function $f(x)$ be invariant with respect to a Weyl group $W$. It is easy to check that the function $\tilde{f}(\lambda)$ is also $W$-invariant. Replace in (8.8) $\lambda$ by $w \lambda, w \in W$, and sum both side of (8.8) over $w \in W$. Then instead of (8.8) we obtain

$$
\tilde{f}(\lambda)=\int_{D_{+}} f(x) \hat{\phi}_{\lambda}(x) d x
$$

where we have taken into account that both $f(x)$ and $\hat{\phi}_{\lambda}(x)$ are $W$-invariant. Note that $\hat{\phi}_{\lambda}(x)$ are modified orbit functions defined by (6.2).

Similarly, starting from (8.9), we obtain the inverse formula:

$$
f(x)=\int_{D_{+}} \tilde{f}(\lambda) \overline{\hat{\phi}_{\lambda}(x)} d \lambda .
$$

For the transforms (8.10) and (8.11) the Plancherel formula

$$
\int_{D_{+}}|f(x)|^{2} d x=\int_{D_{+}}|\tilde{f}(\lambda)|^{2} d \lambda
$$

holds.

\section{$9 \quad$ Finite orbit function transform}

Along with the usual Fourier transform there exists a finite Fourier transform. Similarly, it is possible to introduce a finite orbit function transform. A finite orbit function transform in the one-dimensional case is well known and widely used, it is called the discrete cosine transform (see [35] and references therein). The basis for construction of the finite orbit function transform was given in [9].

This transform is used (see $[15,30]$ and [31]) to find an approximate values of a function $f(x)$ on the whole space $E_{n}$, if its values on some finite set is known.

In order to describe the finite orbit function transform we first consider the finite Fourier transform.

\subsection{Finite Fourier transform}

Let us fix a positive integer $N$ and consider the numbers

$$
e_{m n}:=N^{-1 / 2} \exp (2 \pi \mathrm{i} m n / N), \quad m, n=1,2, \ldots, N .
$$

The matrix $\left(e_{m n}\right)_{m, n=1}^{N}$ is unitary, that is,

$$
\sum_{k} e_{m k} \overline{e_{n k}}=\delta_{m n}, \quad \sum_{k} e_{k m} \overline{e_{k n}}=\delta_{m n}
$$


Indeed, according to the formula for a sum of a geometric progression we have

$$
\begin{aligned}
t^{a}+t^{a+1}+\cdots+t^{a+r} & =(1-t)^{-1} t^{a}\left(1-t^{r+1}\right), \quad t \neq 1, \\
t^{a}+t^{a+1}+\cdots+t^{a+r} & =r+1, \quad t=1 .
\end{aligned}
$$

Setting $t=\exp (2 \pi \mathrm{i}(m-n) / N), a=1$ and $r=N-1$, we prove (9.2).

Let $f(n)$ be a function of $n \in\{1,2, \ldots, N\}$. We may consider the transform

$$
\sum_{n=1}^{N} f(n) e_{m n} \equiv N^{-1 / 2} \sum_{n=1}^{N} f(n) \exp (2 \pi \mathrm{i} m n / N)=\tilde{f}(m) .
$$

Then, since the matrix $\left(e_{m n}\right)_{m, n=1}^{N}$ is unitary, we express $f(n)$ as a linear combination of functions (9.1):

$$
f(n)=N^{-1 / 2} \sum_{m=1}^{N} \tilde{f}(m) \exp (-2 \pi \mathrm{i} m n / N) .
$$

The function $\tilde{f}(m)$ is a finite Fourier transform of $f(n)$. The finite Fourier transform is a linear map. The formula (9.4) gives an inverse transform. The Plancherel formula

$$
\sum_{m=1}^{N}|\tilde{f}(m)|^{2}=\sum_{n=1}^{N}|f(n)|^{2}
$$

holds for transforms (9.3) and (9.4).

The finite Fourier transform on the $r$-dimensional linear space $E_{r}$ is defined in a similar way. We again fix a positive integer $N$. Let $\boldsymbol{m}=\left(m_{1}, m_{2}, \ldots, m_{r}\right)$ be an $r$-tuple of integers such that each $m_{i}$ runs over the integers $1,2, \ldots, N$. Then the finite Fourier transform on $E_{r}$ is given by the kernel

$$
e_{\boldsymbol{m n}}:=e_{m_{1} n_{1}} e_{m_{2} n_{2}} \cdots e_{m_{r} n_{r}}=N^{-r / 2} \exp (2 \pi \mathrm{i} \boldsymbol{m} \cdot \boldsymbol{n} / N)
$$

where $\boldsymbol{m} \cdot \boldsymbol{n}=m_{1} n_{1}+m_{2} n_{2}+\cdots+m_{r} n_{r}$. If $F(\boldsymbol{m})$ is a function of $r$-tuples $\boldsymbol{m}, m_{i} \in\{1,2, \ldots, N\}$, then the finite Fourier transform of $F$ is given by

$$
\tilde{F}(\boldsymbol{n})=N^{-r / 2} \sum_{\boldsymbol{m}} F(\boldsymbol{m}) \exp (2 \pi \mathrm{i} \boldsymbol{m} \cdot \boldsymbol{n} / N) .
$$

The inverse transform is

$$
F(\boldsymbol{m})=N^{-r / 2} \sum_{\boldsymbol{n}} \tilde{F}(\boldsymbol{n}) \exp (-2 \pi \mathrm{i} \boldsymbol{m} \cdot \boldsymbol{n} / N) .
$$

The corresponding Plancherel formula is of the form $\sum_{\boldsymbol{m}}|F(\boldsymbol{m})|^{2}=\sum_{\boldsymbol{n}}|\tilde{F}(\boldsymbol{n})|^{2}$.

\subsection{W-invariant lattices}

In order to determine an analogue of the finite Fourier transform, based on orbit functions, we need a symmetrized analogue of the set

$$
\left\{\boldsymbol{m}=\left\{m_{1}, \ldots, m_{r}\right\} \mid m_{i} \in\{1,2, \ldots, N\}\right\},
$$

used for multidimensional finite Fourier transform. Such a set has to be invariant with respect to the Weyl group $W$. It was constructed in [9]. Let us briefly describe it. 
The lattice $Q^{\vee}$ is a discrete $W$-invariant subset of $E_{n}$. Clearly, the set $\frac{1}{m} Q^{\vee}$ is also $W$-invariant, where $m$ is a fixed positive integer. Then the set

$$
T_{m}=\frac{1}{m} Q^{\vee} / Q^{\vee}
$$

is finite and $W$-invariant. If $\alpha_{1}, \alpha_{2}, \ldots, \alpha_{n}$ is the set of simple roots for the Weyl group $W$, then $T_{m}$ can be identified with the set of elements

$$
\frac{1}{m} \sum_{i=1}^{n} d_{i} \alpha_{i}^{\vee}, \quad d_{i}=0,1,2, \ldots, m-1 .
$$

We need to select from $T_{m}$ a set of elements which belongs to the closure $\bar{F}$ of the fundamental domain $F$. These elements lie in the collection $\frac{1}{m} Q^{\vee} \cap \bar{F}$.

Let $\mu \in \frac{1}{m} Q^{\vee} \cap \bar{F}$ be an element determining an element of $T_{m}$ and let $M$ be the least positive integer such that $M \mu \in P^{\vee}$. (Then there exists a least positive integer $N$ such that $N \mu \in Q^{\vee}$. One has $M \mid N$ and $N \mid m$; see [9].)

The collection of points of $T_{m}$ which belong to $\bar{F}$ (we denote the set of these points by $F_{M}$ ) can be derived from the results of V. Kac in [36]. It coincides with the set of elements

$$
s=\frac{s_{1}}{M} \omega_{1}^{\vee}+\cdots+\frac{s_{n}}{M} \omega_{n}^{\vee}, \quad \omega_{i}^{\vee}:=\frac{2 \omega_{i}}{\left\langle\alpha_{i}, \alpha_{i}\right\rangle},
$$

where $s_{1}, s_{2}, \ldots, s_{n}$ run over the values from $\mathbb{Z}^{\geq}$which satisfy the following condition: there exists a non-negative integer $s_{0}$ such that

$$
s_{0}+\sum_{i=1}^{n} s_{i} m_{i}=M,
$$

where $m_{1}, \ldots, m_{n}$ are positive integers taken from the formula (2.8). (In Subsection 2.3 one can find values of $m_{i}$ for all simple Lie algebras.)

Indeed, the fundamental domain consists of all points $y$ from the dominant Weyl chamber

for which $\langle y, \xi\rangle \leq 1$, where $\xi$ is the highest (long) root, $\xi=\sum_{i=1}^{n} m_{i} \alpha_{i}$. Since $\left\langle\alpha_{i}, \hat{\omega}_{j}\right\rangle=\delta_{i j}$ and for elements $s$ of (9.6) one has $s_{i} / M \geq 0$ and

$$
\langle s, \xi\rangle=\frac{1}{M} \sum_{i=1}^{n} s_{i} m_{i}=\frac{1}{M}\left(M-s_{0}\right) \leq 1,
$$

then $s \in \bar{F}$. The converse reasoning shows that points of $\frac{1}{m} Q^{\vee} \cap \bar{F}$ must be of the form (9.6).

The numbers $s_{0}, s_{1}, s_{2}, \ldots, s_{n}$ can be viewed as attached to the corresponding nodes of the extended Coxeter-Dynkin diagram.

To every positive integer $M$ there corresponds a grid $F_{M}$ of points $(9.6)$ in $\bar{F}$. This grid is related to some set $T_{m}$ such that $M \mid m$. The precise relation between $M$ and $m$ can be defined by the grid $F_{M}$ (see [9]) . Acting upon the grid $F_{M}$ by elements of the Weyl group $W$ we obtain the whole set $T_{m}$.

Remark that for fulfilling decompositions in orbit functions on a finite set we need a grid $F_{M}$ and do not need the number $m$. This number is needed for proving the corresponding results.

\subsection{Expending in orbit functions through finite sets}

The aim of this subsection is to give an analogue of the finite Fourier transform when, instead of exponential functions, we use orbit functions. This analogue is not so simple as the finite 
Fourier transform. For this reason, we consider some weak form of the transform. In fact, we consider this weak form in order to be able to recover the decomposition $f(x)=\sum_{\lambda} a_{\lambda} \phi_{\lambda}(x)$ for all values $x \in E_{n}$ by values of $f(x)$ on a finite set of point.

When considering the finite Fourier transform of Subsection 9.1, we restrict the exponential function to a finite set. Similarly, in order to determine finite orbit function transform we have to restrict orbit functions $\phi_{\lambda}(x)$ to an appropriate finite set of values of $x$. Candidates for such finite sets are sets $T_{m}$. However, orbit functions $\phi_{\lambda}(x), \lambda \in P_{+}$, are invariant with respect to the affine Weyl group $W^{\text {aff }}$. For this reason, we consider these orbit functions $\phi_{\lambda}(x)$ on grids $F_{M}$.

On the other hand, we also have to choose a finite number of orbit functions, that is, a finite number of dominant elements $\lambda \in P_{+}$. The best choice is when the number of orbit functions coincides with $\left|F_{M}\right|$. These orbit functions must be selected in such a way that the matrix

$$
\left(\phi_{\lambda_{i}}\left(x_{j}\right)\right)_{\lambda_{i} \in \Omega, x_{j} \in F_{M}}
$$

(where $\Omega$ is our finite set of dominant elements $\lambda \in P_{+}$) is not singular. In order to have nonsingularity of this matrix some conditions must be satisfied. In general, they are not known. For this reason, we consider some weaker form of the transform (when $|\Omega| \geq\left|F_{M}\right|$ ) and then explain how the set $|\Omega|$ of $\lambda \in P_{+}$can be chosen in such a way that $|\Omega|=\left|F_{M}\right|$.

Let $O(\lambda)$ and $O(\mu)$ be two different $W$-orbits. We say that the group $T_{m}$ separates $O(\lambda)$ and $O(\mu)$ if for any two different elements $\lambda_{1} \in O(\lambda)$ and $\mu_{1} \in O(\mu)$ there exists an element $x \in T_{m}$ such that $\exp \left(2 \pi \mathrm{i}\left\langle\lambda_{1}, x\right\rangle\right) \neq \exp \left(2 \pi \mathrm{i}\left\langle\mu_{1}, x\right\rangle\right)$. Note that $\lambda$ may coincides with $\mu$.

Let $f_{1}$ and $f_{2}$ be two functions on $E_{n}$ which are finite linear combinations of orbit functions. We introduce a $T_{m}$-scalar product by the formula

$$
\left\langle f_{1}, f_{2}\right\rangle_{T_{m}}=\sum_{x \in T_{m}} f_{1}(x) \overline{f_{2}(x)}
$$

Proposition 7. If $T_{m}$ separates $O(\lambda)$ and $O(\mu)$, then

$$
\left\langle\phi_{\lambda}, \phi_{\mu}\right\rangle_{T_{m}}=m^{n}|O(\lambda)| \delta_{\lambda \mu}
$$

Proof. We have

$$
\begin{aligned}
\left\langle\phi_{\lambda}, \phi_{\mu}\right\rangle_{T_{m}} & =\sum_{x \in T_{m}} \sum_{\sigma \in O(\lambda)} \sum_{\tau \in O(\mu)} \exp (2 \pi \mathrm{i}\langle\sigma-\tau, x\rangle) \\
& =\sum_{\sigma \in O(\lambda)} \sum_{\tau \in O(\mu)}\left(\sum_{x \in T_{m}} \exp (2 \pi \mathrm{i}\langle\sigma-\tau, x\rangle)\right) .
\end{aligned}
$$

Since $T_{m}$ separates $O(\lambda)$ and $O(\mu)$, then none of the non-zero differences $\sigma-\tau$ in the last sum vanishes on $T_{m}$. Since $T_{m}$ is a group and $\left|T_{m}\right|=m^{n}$, one has

$$
\sum_{x \in T_{m}} \exp (2 \pi \mathrm{i}\langle\sigma-\tau, x\rangle)=m^{n} \delta_{\sigma \tau}
$$

Then it follows from (9.10) that $\left\langle\phi_{\lambda}, \phi_{\mu}\right\rangle_{T_{m}}=m^{n}|O(\lambda)| \delta_{\lambda \mu}$. The proposition is proved.

Let $f$ be a $W^{\text {aff }}$-invariant function on $E_{n}$ which is a finite linear combination of orbit functions:

$$
f(x)=\sum_{\lambda_{j} \in P_{+}} a_{\lambda_{j}} \phi_{\lambda_{j}}(x) .
$$

Our aim is to determine $f(x), x \in E_{n}$, by its values on a finite subset of $E_{n}$, namely, on $T_{m}$. 
We suppose that $T_{m}$ separates orbits on the right hand side of (9.11). Then taking the $T_{m^{-}}$ scalar product of both sides of (9.11) with $\phi_{\lambda_{i}}$ and taking into account the relation (9.9) we obtain

$$
a_{\lambda_{i}}=\left(m^{n}\left|O\left(\lambda_{i}\right)\right|\right)^{-1}\left\langle f, \phi_{\lambda_{i}}\right\rangle_{T_{m}} .
$$

Let $s^{(1)}, s^{(2)}, \ldots, s^{(h)}$ be all elements of $\bar{F} \cap \frac{1}{m} Q^{\vee}$. As before, by $W_{s^{(i)}}$ we denote the subgroup of $W$ whose elements leave $s^{(i)}$ invariant. Then

$$
\begin{aligned}
a_{\lambda_{j}} & =m^{-n}\left|O\left(\lambda_{j}\right)\right|^{-1} \sum_{x \in T_{m}} f(x) \overline{\phi_{\lambda_{j}}(x)}=m^{-n} \frac{\left|W_{\lambda_{j}}\right|}{|W|} \sum_{i=1}^{h} \frac{|W|}{\left|W_{s^{(i)}}\right|} f\left(s^{(i)}\right) \overline{\phi_{\lambda_{j}}\left(s^{(i)}\right)} \\
& =m^{-n}\left|W_{\lambda_{j}}\right| \sum_{i=1}^{h}\left|W_{s^{(i)}}\right|^{-1} f\left(s^{(i)}\right) \overline{\phi_{\lambda_{j}}\left(s^{(i)}\right)},
\end{aligned}
$$

where $W_{\lambda_{j}}$ is a stabilizer subgroup of $\lambda_{j}$ in $W$.

Thus, a finite number of values $f\left(s^{(i)}\right), i=1,2, \ldots, h$, of the function $f(x)$ determines the coefficients $a_{\lambda_{j}}$ and, therefore, the function $f(x)$ on the whole space $E_{n}$.

This means that we can reconstruct a $W^{\text {aff }}$-invariant function $f(x)$ on the whole space $E_{n}$ by its values on the finite set $F_{M}$ under an appropriate value of $M$. Namely, we have to expand this function, taken on $F_{M}$, into the series (9.11) by means of the coefficients $a_{\lambda_{j}}$, determined by formula (9.12), and then to continue analytically the expansion (9.11) to the whole fundamental domain $\bar{F}$ (and, therefore, to the whole space $E_{n}$ ), that is, to consider the decomposition (9.11) for all $x \in E_{n}$.

We have assumed that the function $f(x)$ is a finite linear combination of orbit functions. If $f(x)$ expands into infinite sum of orbit functions, then for applying the above procedure we have to approximate the function $f(x)$ by taking a finite number of terms in this infinite sum and then apply the procedure. That is, in this case we obtain an approximate expression of the function $f(x)$ by using a finite number of its values.

At last, we explain how to choose a set $\Omega$ in formula (9.8). The set $F_{M}$ consists of the points (9.6). This set determines the set of points

$$
\lambda=s_{1} \omega_{1}+\cdots+s_{n} \omega_{n}
$$

where $s_{1}, \ldots, s_{n}$ run over the same values as for the set $F_{M}$. The set of these weights can be taken as the set $\Omega$. The corresponding considerations for rank 2 cases can be seen in [30] and [31].

\subsection{Orbit functions at rational points}

In the fundamental domain $F$ there exist a finite number of points (we denote them $x_{j}$ ) such that all orbit functions take integer values at these points:

$$
\phi_{\lambda}\left(x_{j}\right) \in \mathbb{Z} \quad \text { for all } \quad \lambda \in P .
$$

Elements of a Lie group, corresponding to these points (see Subsection 8.1), are called rational [37].

More generally, in each compact simple Lie group there exist few conjugacy classes of elements, which have all integer-valued characters (hence also integer-valued orbit functions). Points of these conjugacy classes are rational elements. Their orders $M$ are relatively low in the Lie group. The points of the fundamental domain, representing such elements, are listed 
in Tables 1-6 for compact simple Lie groups of rank 2 and 3. Generally such points are not found in the literature except for the group $E_{8}$, see [38].

The simplest example of a rational element in a compact simple Lie group is the identity element of the group. The character at the identity element is equal to the dimension of the corresponding representation; the orbit function at the identity element is equal to the size of the corresponding Weyl group orbit.

Lines of Tables 1-6 identify (conjugacy classes of) rational elements of the Lie group. The first entry $M$ at each line is the adjoint order of the element, i.e. its order in the adjoint representation of the group or, more generally, in any representation where the center of the Lie group coalesces to identity.

The second entry $N$ on a line is the full order of the element, that is its order in representations where the center of the Lie group is faithfully represented. $M$ always divides $N$ and $1 \leq \frac{N}{M} \leq|Z|$, where $|Z|$ is the order of the center of the compact simple Lie group.

There is an interesting general one-to-one correspondence between rational elements of $A_{2 k-1}$ and of $A_{2 k}$, which we illustrate in Tables 1 and 4 .

Table 1. Rational elements in $A_{2}$ and in $A_{1}$, their adjoint orders $M$ and full orders $N$.

\begin{tabular}{|c|c||c|c||c|c||c|c|}
\hline$M$ & $N$ & {$\left[s_{0}, s_{1}, s_{2}\right]$} & $\left(\frac{s_{1}}{M}, \frac{s_{2}}{M}\right)$ & $M$ & $N$ & {$\left[s_{0}, s_{1}\right]$} & $\left(\frac{s_{1}}{M}\right)$ \\
\hline \hline 1 & 1 & {$[1,0,0]$} & $(0,0)$ & 1 & 1 & {$[1,0]$} & $(0)$ \\
\hline 2 & 2 & {$[0,1,1]$} & $\left(\frac{1}{2}, \frac{1}{2}\right)$ & 1 & 2 & {$[0,1]$} & $\left(\frac{1}{2}\right)$ \\
\hline 3 & 3 & {$[1,1,1]$} & $\left(\frac{1}{3}, \frac{1}{3}\right)$ & 3 & 3 & {$[1,2]$} & $\left(\frac{2}{3}\right)$ \\
\hline 4 & 4 & {$[2,1,1]$} & $\left(\frac{1}{4}, \frac{1}{4}\right)$ & 2 & 4 & {$[1,1]$} & $\left(\frac{1}{4}\right)$ \\
\hline 6 & 6 & {$[4,1,1]$} & $\left(\frac{1}{6}, \frac{1}{6}\right)$ & 3 & 6 & {$[2,1]$} & $\left(\frac{1}{6}\right)$ \\
\hline
\end{tabular}

Table 2. Rational elements in $C_{2}$, their adjoint order $M=s_{0}+2 s_{1}+s_{2}$ and full order $N$.

\begin{tabular}{|c|c||c|c|}
\hline$M$ & $N$ & {$\left[s_{0}, s_{1}, s_{2}\right]$} & $\left(\frac{s_{1}}{M}, \frac{s_{2}}{M}\right)$ \\
\hline \hline 1 & 1 & {$[1,0,0]$} & $(0,0)$ \\
\hline 1 & 2 & {$[0,0,1]$} & $(0,1)$ \\
\hline \hline 2 & 2 & {$[0,1,0]$} & $\left(\frac{1}{2}, 0\right)$ \\
\hline 2 & 4 & {$[1,0,1]$} & $\left(0, \frac{1}{2}\right)$ \\
\hline \hline 3 & 3 & {$[1,0,2]$} & $\left(0, \frac{2}{3}\right)$ \\
\hline 3 & 6 & {$[2,0,1]$} & $\left(0, \frac{1}{3}\right)$ \\
\hline 3 & 6 & {$[0,1,1]$} & $\left(\frac{1}{3}, \frac{1}{3}\right)$ \\
\hline \hline 4 & 4 & {$[2,1,0]$} & $\left(\frac{1}{4}, 0\right)$ \\
\hline 4 & 4 & {$[0,1,2]$} & $\left(\frac{1}{4}, \frac{1}{2}\right)$ \\
\hline 4 & 8 & {$[1,1,1]$} & $\left(\frac{1}{4}, \frac{1}{4}\right)$ \\
\hline \hline 5 & 5 & {$[1,1,2]$} & $\left(\frac{1}{5}, \frac{2}{5}\right)$ \\
\hline 5 & 10 & {$[2,1,1]$} & $\left(\frac{1}{5}, \frac{1}{5}\right)$ \\
\hline \hline 6 & 6 & {$[4,1,0]$} & $\left(\frac{1}{6}, 0\right)$ \\
\hline 6 & 6 & {$[2,1,2]$} & $\left(\frac{1}{6}, \frac{1}{3}\right)$ \\
\hline 6 & 6 & {$[0,1,4]$} & $\left(\frac{1}{6}, \frac{2}{3}\right)$ \\
\hline 6 & 12 & {$[1,2,1]$} & $\left(\frac{1}{3}, \frac{1}{6}\right)$ \\
\hline \hline 12 & 12 & {$[6,1,4]$} & $\left(\frac{1}{12}, \frac{1}{3}\right)$ \\
\hline 12 & 12 & {$[4,1,6]$} & $\left(\frac{1}{12}, \frac{1}{2}\right)$ \\
\hline
\end{tabular}


Table 3. Rational elements in $G_{2}$, their adjoint order $M=s_{0}+2 s_{1}+3 s_{2}$ and the full order $N$.

\begin{tabular}{|c|c||c|c|}
\hline$M$ & $N$ & {$\left[s_{0}, s_{1}, s_{2}\right]$} & $\left(\frac{s_{1}}{M}, \frac{s_{2}}{M}\right)$ \\
\hline \hline 1 & 1 & {$[1,0,0]$} & $(0,0)$ \\
\hline \hline 2 & 2 & {$[0,1,0]$} & $\left(\frac{1}{2}, 0\right)$ \\
\hline \hline 3 & 3 & {$[1,1,0]$} & $\left(\frac{1}{3}, 0\right)$ \\
\hline 3 & 3 & {$[0,0,1]$} & $\left(0, \frac{1}{3}\right)$ \\
\hline \hline 4 & 4 & {$[2,1,0]$} & $\left(\frac{1}{4}, 0\right)$ \\
\hline 4 & 4 & {$[1,0,1]$} & $\left(0, \frac{1}{4}\right)$ \\
\hline \hline 6 & 6 & {$[4,1,0]$} & $\left(\frac{1}{6}, 0\right)$ \\
\hline 6 & 6 & {$[3,0,1]$} & $\left(0, \frac{1}{6}\right)$ \\
\hline 6 & 6 & {$[1,1,1]$} & $\left(\frac{1}{6}, \frac{1}{6}\right)$ \\
\hline \hline 7 & 7 & {$[2,1,1]$} & $\left(\frac{1}{7}, \frac{1}{7}\right)$ \\
\hline \hline 8 & 8 & {$[3,1,1]$} & $\left(\frac{1}{8}, \frac{1}{8}\right)$ \\
\hline 8 & 8 & {$[1,2,1]$} & $\left(\frac{1}{4}, \frac{1}{8}\right)$ \\
\hline \hline 12 & 12 & {$[3,3,1]$} & $\left(\frac{1}{4}, \frac{1}{12}\right)$ \\
\hline 12 & 12 & {$[1,4,1]$} & $\left(\frac{1}{3}, \frac{1}{12}\right)$ \\
\hline
\end{tabular}

Table 4. Rational elements in $A_{3}$ and in $A_{4}$, their adjoint orders $M$ and the full orders $N$.

\begin{tabular}{|c|c||c|c||c|c||c|c|}
\hline$M$ & $N$ & {$\left[s_{0}, s_{1}, s_{2}, s_{3}\right]$} & $\left(\frac{s_{1}}{M}, \frac{s_{2}}{M}, \frac{s_{3}}{M}\right)$ & $M$ & $N$ & {$\left[s_{0}, s_{1}, s_{2}, s_{3}, s_{4}\right]$} & $\left(\frac{s_{1}}{M}, \frac{s_{2}}{M}, \frac{s_{3}}{M}, \frac{s_{2}}{M}\right)$ \\
\hline \hline 1 & 1 & {$[1,0,0,0]$} & $(0,0,0)$ & 1 & 1 & {$[1,0,0,0,0]$} & $(0,0,0,0)$ \\
\hline 1 & 2 & {$[0,0,1,0]$} & $(0,1,0)$ & 2 & 2 & {$[0,0,1,1,0]$} & $\left(0, \frac{1}{2}, \frac{1}{2}, 0\right)$ \\
\hline \hline 2 & 2 & {$[0,1,0,1]$} & $\left(\frac{1}{2}, 0, \frac{1}{2}\right)$ & 2 & 2 & {$[0,1,0,0,1]$} & $\left(\frac{1}{2}, 0,0, \frac{1}{2}\right)$ \\
\hline 2 & 4 & {$[1,0,1,0]$} & $\left(0, \frac{1}{2}, 0\right)$ & 4 & 4 & {$[2,0,1,1,0]$} & $\left(0, \frac{1}{4}, \frac{1}{4}, 0\right)$ \\
\hline \hline 3 & 3 & {$[1,0,2,0]$} & $\left(0, \frac{2}{3}, 0\right)$ & 3 & 3 & {$[1,0,1,1,0]$} & $\left(0, \frac{1}{3}, \frac{1}{3}, 0\right)$ \\
\hline 3 & 3 & {$[1,1,0,1]$} & $\left(\frac{1}{3}, 0, \frac{1}{3}\right)$ & 3 & 3 & {$[1,1,0,0,1]$} & $\left(\frac{1}{3}, 0,0, \frac{1}{3}\right)$ \\
\hline 3 & 6 & {$[2,0,1,0]$} & $\left(0, \frac{1}{3}, 0\right)$ & 6 & 6 & {$[4,0,1,1,0]$} & $\left(0, \frac{1}{6}, \frac{1}{6}, 0\right)$ \\
\hline 3 & 6 & {$[0,1,1,1]$} & $\left(\frac{1}{3}, \frac{1}{3}, \frac{1}{3}\right)$ & 6 & 6 & {$[0,2,1,1,2]$} & $\left(\frac{1}{3}, \frac{1}{6}, \frac{1}{6}, \frac{1}{3}\right)$ \\
\hline \hline 4 & 4 & {$[2,1,0,1]$} & $\left(\frac{1}{4}, 0, \frac{1}{4}\right)$ & 4 & 4 & {$[2,1,0,0,1]$} & $\left(\frac{1}{4}, 0,0, \frac{1}{4}\right)$ \\
\hline 4 & 4 & {$[0,1,2,1]$} & $\left(\frac{1}{4}, \frac{1}{2}, \frac{1}{4}\right)$ & 4 & 4 & {$[0,1,1,1,1]$} & $\left(\frac{1}{4}, \frac{1}{4}, \frac{1}{4}, \frac{1}{4}\right)$ \\
\hline 4 & 8 & {$[1,1,1,1]$} & $\left(\frac{1}{4}, \frac{1}{4}, \frac{1}{4}\right)$ & 8 & 8 & {$[2,2,1,1,2]$} & $\left(\frac{1}{4}, \frac{1}{8}, \frac{1}{8}, \frac{1}{4}\right)$ \\
\hline \hline 5 & 5 & {$[1,1,2,1]$} & $\left(\frac{1}{5}, \frac{2}{5}, \frac{1}{5}\right)$ & 5 & 5 & {$[1,1,1,1,1]$} & $\left(\frac{1}{5}, \frac{1}{5}, \frac{1}{5}, \frac{1}{5}\right)$ \\
\hline 5 & 10 & {$[2,1,1,1]$} & $\left(\frac{1}{5}, \frac{1}{5}, \frac{1}{5}\right)$ & 10 & 10 & {$[4,2,1,1,2]$} & $\left(\frac{1}{5}, \frac{1}{10}, \frac{1}{10}, \frac{1}{5}\right)$ \\
\hline \hline 6 & 6 & {$[4,1,0,1]$} & $\left(\frac{1}{6}, 0, \frac{1}{6}\right)$ & 6 & 6 & {$[4,1,0,0,1]$} & $\left(\frac{1}{6}, 0,0, \frac{1}{6}\right)$ \\
\hline 6 & 6 & {$[2,1,2,1]$} & $\left(\frac{1}{6}, \frac{1}{3}, \frac{1}{6}\right)$ & 6 & 6 & {$[2,1,1,1,1]$} & $\left(\frac{1}{6}, \frac{1}{6}, \frac{1}{6}, \frac{1}{6}\right)$ \\
\hline 6 & 6 & {$[0,1,4,1]$} & $\left(\frac{1}{6}, \frac{2}{3}, \frac{1}{6}\right)$ & 6 & 6 & {$[0,1,2,2,1]$} & $\left(\frac{1}{6}, \frac{1}{3}, \frac{1}{3}, \frac{1}{6}\right)$ \\
\hline 6 & 12 & {$[1,2,1,2]$} & $\left(\frac{1}{3}, \frac{1}{6}, \frac{1}{3}\right)$ & 12 & 12 & {$[2,4,1,1,4]$} & $\left(\frac{1}{3}, \frac{1}{12}, \frac{1}{12}, \frac{1}{3}\right)$ \\
\hline \hline 12 & 12 & {$[6,1,4,1]$} & $\left(\frac{1}{12}, \frac{1}{3}, \frac{1}{12}\right)$ & 12 & 12 & {$[6,1,2,2,1]$} & $\left(\frac{1}{12}, \frac{1}{6}, \frac{1}{6}, \frac{1}{12}\right)$ \\
\hline 12 & 12 & {$[4,1,6,1]$} & $\left(\frac{1}{12}, \frac{1}{2}, \frac{1}{12}\right)$ & 12 & 12 & {$[4,1,3,3,1]$} & $\left(\frac{1}{12}, \frac{1}{4}, \frac{1}{4}, \frac{1}{12}\right)$ \\
\hline \hline
\end{tabular}


Table 5. Rational elements in $B_{3}$, their adjoint order $M=s_{0}+s_{1}+2 s_{2}+2 s_{3}$ and the full order $N$.

\begin{tabular}{|c|c|c|c|}
\hline$M$ & $N$ & {$\left[s_{0}, s_{1}, s_{2}, s_{3}\right]$} & $\left(\frac{s_{1}}{M}, \frac{s_{2}}{M}, \frac{s_{3}}{M}\right)$ \\
\hline 1 & 1 & {$[1,0,0,0]$} & $(0,0,0)$ \\
\hline 1 & 2 & {$[0,1,0,0]$} & $(1,0,0)$ \\
\hline 2 & $\overline{2}$ & {$[0,0,1,0]$} & $\left(0, \frac{1}{2}, 0\right)$ \\
\hline 2 & 4 & {$[1,1,0,0]$} & $\left(\frac{1}{2}, 0,0\right)$ \\
\hline 2 & 4 & {$[0,0,0,1]$} & $\left(0,0, \frac{1}{2},\right)$ \\
\hline 3 & 3 & {$[1,2,0,0]$} & $\left(\frac{2}{3}, 0,0\right)$ \\
\hline 3 & 3 & {$[1,0,1,0]$} & $\left(0, \frac{1}{3}, 0\right.$ \\
\hline 3 & 3 & {$[0,1,0,1]$} & $\left(\frac{1}{3}, 0, \frac{1}{3}\right)$ \\
\hline 3 & 6 & {$[2,1,0,0]$} & $\left(\frac{1}{3}, 0,0\right)$ \\
\hline 3 & 6 & {$[0,1,1,0]$} & $\left(\frac{1}{3}, \frac{1}{3}, 0\right)$ \\
\hline 3 & 6 & {$[1,0,0,1]$} & $\left(0,0, \frac{1}{3}\right)$ \\
\hline 4 & 4 & {$[2,0,1,0]$} & $\left(0, \frac{1}{4}, 0\right)$ \\
\hline 4 & 4 & {$[0,2,1,0]$} & $\left(\frac{1}{2}, \frac{1}{4}, 0\right)$ \\
\hline 4 & 4 & {$[1,1,0,1]$} & $\left(\frac{1}{4}, 0, \frac{1}{4}\right)$ \\
\hline 4 & 8 & {$[1,1,1,0]$} & $\left(\frac{1}{4}, \frac{1}{4}, 0\right)$ \\
\hline 4 & 8 & {$[0,0,1,1]$} & $\left(0, \frac{1}{4}, \frac{1}{4}\right)$ \\
\hline 5 & 5 & {$[1,2,1,0]$} & $\left(\frac{2}{5}, \frac{1}{5}, 0\right)$ \\
\hline 5 & 10 & {$[2,1,1,0]$} & $\left(\frac{1}{5}, \frac{1}{5}, 0\right)$ \\
\hline 6 & 6 & {$[4,0,1,0]$} & $\left(0, \frac{1}{6}, 0\right)$ \\
\hline 6 & 6 & {$[2,2,1,0]$} & $\left(\frac{1}{3}, \frac{1}{6}, 0\right)$ \\
\hline 6 & 6 & {$[0,4,1,0]$} & $\left(\frac{2}{3}, \frac{1}{6}, 0\right)$ \\
\hline 6 & 6 & {$[3,1,0,1]$} & $\left(\frac{1}{6}, 0, \frac{1}{6}\right)$ \\
\hline 6 & 6 & {$[1,3,0,1]$} & $\left(\frac{1}{2}, 0, \frac{1}{6}\right)$ \\
\hline 6 & 6 & {$[1,1,1,1]$} & $\left(\frac{1}{6}, \frac{1}{6}, \frac{1}{6}\right)$ \\
\hline 6 & 6 & {$[0,0,1,2]$} & $\left(0, \frac{1}{6}, \frac{1}{3}\right)$ \\
\hline 6 & 12 & {$[1,1,2,0]$} & $\left(\frac{1}{6}, \frac{1}{3}, 0\right)$ \\
\hline 6 & 12 & {$[2,2,0,1]$} & $\left(\frac{1}{3}, 0, \frac{1}{6}\right)$ \\
\hline 6 & 12 & {$[0,0,2,1]$} & $\left(0, \frac{1}{3}, \frac{1}{6}\right)$ \\
\hline 6 & 12 & {$[1,1,0,2]$} & $\left(\frac{1}{6}, 0, \frac{1}{3}\right)$ \\
\hline 7 & 7 & {$[2,1,1,1]$} & $\left(\frac{1}{7}, \frac{1}{7}, \frac{1}{7}\right)$ \\
\hline 7 & 14 & {$[1,2,1,1]$} & $\left(\frac{2}{7}, \frac{1}{7}, \frac{1}{7}\right)$ \\
\hline 8 & 8 & {$[3,1,1,1]$} & $\left.\frac{1}{3}, \frac{1}{8}, \frac{1}{8}\right)$ \\
\hline 8 & 8 & {$[1,3,1,1]$} & $\left.\frac{1}{2}, \frac{1}{8}\right)$ \\
\hline 8 & 8 & {$[1,1,2,1]$} & $\left.\frac{1}{8}\right)$ \\
\hline 9 & 9 & {$[2,3,1,1]$} & $\left(\frac{1}{3}, \frac{1}{9}, \frac{1}{9}\right)$ \\
\hline 9 & 18 & {$[3,2,1,1]$} & $\left(\frac{2}{9}, \frac{1}{9}, \frac{1}{9}\right)$ \\
\hline 10 & 20 & {$[2,2,2,1]$} & $\left.\frac{1}{5}, \frac{1}{5}, \frac{1}{10}\right)$ \\
\hline 10 & 20 & {$[1,1,2,2]$} & $\left(\frac{1}{10}, \frac{1}{5}, \frac{1}{5}\right)$ \\
\hline 12 & 12 & {$[6,4,1,0]$} & $\left(\frac{1}{3}, \frac{1}{12}, 0\right)$ \\
\hline 12 & 12 & {$[4,6,1,0]$} & $\left.\frac{1}{2}, \frac{1}{12}, 0\right)$ \\
\hline 12 & 12 & {$[3,1,3,1]$} & $\left(\frac{1}{12}, \frac{1}{4}, \frac{1}{12}\right)$ \\
\hline 12 & 12 & {$[1,3,3,1]$} & $\left(\frac{1}{4}, \frac{1}{4}, \frac{1}{12}\right)$ \\
\hline 12 & 12 & {$[1,1,4,1]$} & $\left(\frac{1}{12}, \frac{1}{3}, \frac{1}{12}\right)$ \\
\hline 12 & 12 & {$[5,1,0,3]$} & $\left(\frac{1}{12}, 0, \frac{1}{4}\right)$ \\
\hline 12 & 12 & {$[1,5,0,3]$} & $\left(\frac{5}{12}, 0, \frac{1}{4}\right)$ \\
\hline 12 & 24 & {$[3,3,1,2]$} & $\left(\frac{1}{4}, \frac{1}{12}, \frac{1}{6}\right)$ \\
\hline 12 & 24 & {$[2,2,1,3]$} & $\left(\frac{1}{6}, \frac{1}{12}, \frac{1}{4}\right)$ \\
\hline$\overline{15}$ & $\overline{15}$ & {$[4,1,2,3]$} & $\left.\frac{2}{15}, \frac{1}{5}\right)$ \\
\hline 15 & 30 & {$[1,4,2,3]$} & $\left(\frac{4}{15}, \frac{2}{15}, \frac{1}{5}\right)$ \\
\hline
\end{tabular}


Table 6. Rational elements in $C_{3}$, their adjoint order $M=s_{0}+2 s_{1}+2 s_{2}+s_{3}$ and the full order $N$.

\begin{tabular}{|c|c|c|c|}
\hline$M$ & $N$ & {$\left[s_{0}, s_{1}, s_{2}, s_{3}\right]$} & $\left(\frac{s_{1}}{M}, \frac{s_{2}}{M}, \frac{s_{3}}{M}\right)$ \\
\hline 1 & 1 & {$[1,0,0,0]$} & $(0,0,0)$ \\
\hline 1 & 2 & {$[0,0,0,1]$} & $(0,0,1)$ \\
\hline 2 & 2 & {$[0,1,0,0]$} & $\left(\frac{1}{2}, 0,0\right)$ \\
\hline 2 & 2 & {$[0,0,1,0]$} & $\left(0, \frac{1}{2}, 0\right)$ \\
\hline 2 & 4 & {$[1,0,0,1]$} & $\left(0,0, \frac{1}{2},\right)$ \\
\hline 3 & 3 & {$[1,1,0,0]$} & $\left(\frac{1}{3}, 0,0\right)$ \\
\hline 3 & 3 & {$[1,0,1,0]$} & $\left(0, \frac{1}{3}, 0\right.$, \\
\hline 3 & 3 & {$[1,0,0,2]$} & $\left(0,0, \frac{2}{3}\right)$ \\
\hline 3 & 6 & {$[2,0,0,1]$} & $\left(0,0, \frac{1}{3}\right)$ \\
\hline 3 & 6 & {$[0,1,0,1]$} & $\left(\frac{1}{3}, 0, \frac{1}{3}\right)$ \\
\hline 3 & 6 & {$[0,0,1,1]$} & $\left(0, \frac{1}{3}, \frac{1}{3}\right)$ \\
\hline 4 & 4 & {$[2,1,0,0]$} & $\left(\frac{1}{4}, 0,0\right)$ \\
\hline 4 & 4 & {$[2,0,1,0]$} & $\left(0, \frac{1}{4}, 0\right)$ \\
\hline 4 & 4 & {$[0,1,1,0]$} & $\left(\frac{1}{4}, \frac{1}{4}, 0\right)$ \\
\hline 4 & 4 & {$[0,1,0,2]$} & $\left(\frac{1}{4}, 0, \frac{1}{2}\right)$ \\
\hline 4 & 4 & {$[0,0,1,2]$} & $\left(0, \frac{1}{4}, \frac{1}{2}\right)$ \\
\hline 5 & 5 & {$[1,1,1,0]$} & $\left(\frac{1}{5}, \frac{1}{5}, 0\right)$ \\
\hline 5 & 10 & {$[0,1,1,1]$} & $\left(\frac{1}{5}, \frac{1}{5}, \frac{1}{5}\right)$ \\
\hline 6 & 6 & {$[4,1,0,0]$} & $\left(\frac{1}{6}, 0,0\right)$ \\
\hline 6 & 6 & {$[4,0,1,0]$} & $\left(0, \frac{1}{6}, 0\right)$ \\
\hline 6 & 6 & {$[2,1,1,0]$} & $\left(\frac{1}{6}, \frac{1}{6}, 0\right)$ \\
\hline 6 & 6 & {$[0,2,1,0]$} & $\left(\frac{1}{3}, \frac{1}{6}, 0,\right)$ \\
\hline 6 & 6 & {$[0,1,2,0]$} & $\left.\frac{1}{3}, 0, \quad\right)$ \\
\hline 6 & 6 & {$[2,1,0,2]$} & $\left(\frac{1}{6}, 0, \frac{1}{3}\right)$ \\
\hline 6 & 6 & {$[2,0,1,2]$} & $\left(0, \frac{1}{6}, \frac{1}{3}\right)$ \\
\hline 6 & 6 & {$[0,1,1,2]$} & $\left(\frac{1}{6}, \frac{1}{6}, \frac{1}{3}\right)$ \\
\hline 6 & 6 & {$[0,1,0,4]$} & $\left(\frac{1}{6}, 0, \frac{2}{3}\right)$ \\
\hline 6 & 6 & {$[0,0,1,4]$} & $\left(0, \frac{1}{6}, \frac{2}{3}\right)$ \\
\hline 6 & 12 & {$[1,1,1,1]$} & $\left(\frac{1}{6}, \frac{1}{6}, \frac{1}{6}\right)$ \\
\hline 7 & 7 & {$[1,1,1,2]$} & $\left(\frac{1}{7}, \frac{1}{7}, \frac{2}{7}\right)$ \\
\hline 7 & 14 & {$[2,1,1,1]$} & $\left(\frac{1}{7}, \frac{1}{7}, \frac{1}{7}\right)$ \\
\hline 8 & 8 & {$[2,2,1,0]$} & $\left(\frac{1}{4}, \frac{1}{8}, 0\right)$ \\
\hline 8 & 8 & {$[2,1,1,2]$} & $\left.\frac{1}{3}, \frac{1}{4}\right)$ \\
\hline 8 & 8 & {$[0,1,2,2]$} & $\left(\frac{1}{8}, \frac{1}{4}, \frac{1}{4}\right)$ \\
\hline 9 & 9 & {$[1,2,1,2]$} & $\left(\frac{2}{9}, \frac{1}{9}, \frac{2}{9}\right)$ \\
\hline 9 & 18 & {$[2,1,2,1]$} & $\left(\frac{1}{9}, \frac{2}{9}, \frac{1}{9}\right)$ \\
\hline 10 & 10 & {$[4,2,1,0]$} & $\left(\frac{1}{5}, \frac{1}{10}, 0\right)$ \\
\hline 10 & 10 & {$[0,1,2,4]$} & $\left(\frac{1}{10}, \frac{1}{5}, \frac{2}{5}\right)$ \\
\hline 12 & 12 & {$[2,4,1,0]$} & $\left(\frac{1}{3}, \frac{1}{12}, 0\right)$ \\
\hline 12 & 12 & {$[6,1,2,0]$} & $\left(\frac{1}{12}, \frac{1}{6}, 0\right)$ \\
\hline 12 & 12 & {$[4,1,3,0]$} & $\left(\frac{1}{12}, \frac{1}{4}, 0\right)$ \\
\hline 12 & 12 & {$[2,3,1,2]$} & $\left(\frac{1}{4}, \frac{1}{12}, \frac{1}{6}\right)$ \\
\hline 12 & 12 & {$[2,1,3,2]$} & $\left(\frac{1}{12}, \frac{1}{4}, \frac{1}{6}\right)$ \\
\hline 12 & 12 & {$[0,1,4,2]$} & $\left(\frac{1}{12}, \frac{1}{3}, \frac{1}{6}\right)$ \\
\hline 12 & 12 & {$[6,1,0,4]$} & $\left(\frac{1}{12}, 0, \frac{1}{3}\right)$ \\
\hline 12 & 12 & {$[6,0,1,4]$} & $\left(0, \frac{1}{12}, \frac{1}{3}\right)$ \\
\hline 12 & 12 & {$[4,1,1,4]$} & $\left(\frac{1}{12}, \frac{1}{12}, \frac{1}{3}\right)$ \\
\hline 12 & 12 & {$[0,3,1,4]$} & $\left(\frac{1}{4}, \frac{1}{12}, \frac{1}{3}\right)$ \\
\hline 12 & 12 & {$[4,1,0,6]$} & $\left(\frac{1}{12}, 0, \frac{1}{2}\right)$ \\
\hline
\end{tabular}


Table 6. Continuation.

\begin{tabular}{|c|c||c|c|}
\hline$M$ & $N$ & {$\left[s_{0}, s_{1}, s_{2}, s_{3}\right]$} & $\left(\frac{s_{1}}{M}, \frac{s_{2}}{M}, \frac{s_{3}}{M}\right)$ \\
\hline \hline 12 & 12 & {$[4,0,1,6]$} & $\left(0, \frac{1}{12}, \frac{1}{2}\right)$ \\
\hline 12 & 12 & {$[0,2,1,6]$} & $\left(\frac{1}{6}, \frac{1}{12}, \frac{1}{2}\right)$ \\
\hline \hline 15 & 15 & {$[3,1,2,6]$} & $\left(\frac{1}{15}, \frac{2}{15}, \frac{6}{15}\right)$ \\
\hline 15 & 30 & {$[6,2,1,3]$} & $\left(\frac{2}{15}, \frac{1}{15}, \frac{1}{5}\right)$ \\
\hline \hline 20 & 20 & {$[8,1,3,4]$} & $\left(\frac{1}{20}, \frac{3}{20}, \frac{1}{5}\right)$ \\
\hline 20 & 20 & {$[4,3,1,8]$} & $\left(\frac{3}{20}, \frac{1}{20}, \frac{2}{5}\right)$ \\
\hline \hline 24 & 24 & {$[6,5,1,6]$} & $\left(\frac{5}{24}, \frac{1}{24}, \frac{1}{4}\right)$ \\
\hline 24 & 24 & {$[6,1,5,6]$} & $\left(\frac{1}{24}, \frac{5}{24}, \frac{1}{4}\right)$ \\
\hline \hline 30 & 30 & {$[10,1,6,6]$} & $\left(\frac{1}{30}, \frac{1}{5}, \frac{1}{5}\right)$ \\
\hline 30 & 30 & {$[6,6,1,10]$} & $\left(\frac{1}{5}, \frac{1}{30}, \frac{1}{3}\right)$ \\
\hline \hline
\end{tabular}

\section{Solutions of the Neumann boundary value problem on $n$-dimensional simplexes}

\subsection{The case of $n$-dimensional simplexes related to $A_{n}, B_{n}, C_{n}$ and $D_{n}$}

Let $F$ be the fundamental domain of one of the affine Weyl groups $W^{\text {aff }}\left(A_{n}\right), W^{\text {aff }}\left(B_{n}\right)$, $W^{\text {aff }}\left(C_{n}\right), W^{\text {aff }}\left(D_{n}\right)$. We use the orthogonal coordinates $x_{1}, x_{2}, \ldots, x_{n+1}$ in $F$ in the case of $W^{\text {aff }}\left(A_{n}\right)$ and the orthogonal coordinates $x_{1}, x_{2}, \ldots, x_{n}$ in other cases (see Section 3 ). Thus the fundamental domain $F$ for $W^{\text {aff }}\left(A_{n}\right)$ is placed in the hyperplane $x_{1}+x_{2}+\cdots+x_{n+1}=0$.

We consider the Laplace operator

$$
\Delta=\frac{\partial^{2}}{\partial x_{1}^{2}}+\frac{\partial^{2}}{\partial x_{2}^{2}}+\cdots+\frac{\partial^{2}}{\partial x_{r}^{2}}
$$

on $F$, where $r=n+1$ for $A_{n}$ and $r=n$ for $B_{n}, C_{n}$ and $D_{n}$. Let us take a summand from the expression (6.13) for the orbit function $\phi_{\lambda}(x)$ of $B_{n}$ and act upon it by the operator $\Delta$. We get

$$
\begin{aligned}
& \Delta e^{2 \pi \mathrm{i}\left((w(\varepsilon \lambda))_{1} x_{1}+\cdots+(w(\varepsilon \lambda))_{n} x_{n}\right)}=-4 \pi^{2}\left[\left(\varepsilon_{1} m_{1}\right)^{2}+\cdots+\left(\varepsilon_{n} m_{n}\right)^{2}\right] e^{2 \pi \mathrm{i}\left((w(\varepsilon \lambda))_{1} x_{1}+\cdots+(w(\varepsilon \lambda))_{n} x_{n}\right)} \\
& =-4 \pi^{2}\left(m_{1}^{2}+\cdots+m_{n}^{2}\right) e^{2 \pi \mathrm{i}\left((w(\varepsilon \lambda))_{1} x_{1}+\cdots+(w(\varepsilon \lambda))_{n} x_{n}\right)} \\
& =-4 \pi^{2}\langle\lambda, \lambda\rangle e^{2 \pi \mathrm{i}\left((w(\varepsilon \lambda))_{1} x_{1}+\cdots+(w(\varepsilon \lambda))_{n} x_{n}\right)}
\end{aligned}
$$

where $\lambda=\left(m_{1}, m_{2}, \ldots, m_{n}\right)$ is the weight, determining $\phi_{\lambda}(x)$, in the orthogonal coordinates and $w \in S_{n} / S_{\lambda}$. Since this action does not depend on a summand from (6.13), we have

$$
\Delta \phi_{\lambda}(x)=-4 \pi^{2}\langle\lambda, \lambda\rangle \phi_{\lambda}(x) \text {. }
$$

For $A_{n}, C_{n}$ and $D_{n}$ this formula also holds and the corresponding proofs are the same. Remark that in the case $A_{n}$ the scalar product $\langle\lambda, \lambda\rangle$ is equal to

$$
\langle\lambda, \lambda\rangle=m_{1}^{2}+m_{2}^{2}+\cdots+m_{n+1}^{2} .
$$

Thus, orbit functions are eigenfunctions of the Laplace operator on the fundamental domain $F$ satisfying the Neumann boundary condition

$$
\left.\frac{\partial \phi_{\lambda}(x)}{\partial m}\right|_{\partial F}=0, \quad \lambda \in P_{+},
$$

where $\partial F$ is the $(n-1)$-dimensional boundary of $F$ and $m$ is the normal to the boundary. 


\subsection{The Laplace operator in the $\omega$-basis}

Now we parametrize elements of $F$ by the coordinates in the $\omega$-basis: $x=\theta_{1} \omega_{1}+\cdots+\theta_{2} \omega_{2}$. Denoting by $\partial_{k}$ the partial derivative with respect to $\theta_{k}$, we have the Laplace operator $\Delta$ in the form

$$
\Delta=\sum_{i, j=1}^{n}\left\langle\alpha_{i} \mid \alpha_{i}\right\rangle^{-1} M_{i j} \partial_{i} \partial_{j},
$$

where $\left(M_{i j}\right)$ is the corresponding Cartan matrix. One can see that it is indeed the Laplace operator as follows. The matrix $\left(S_{i j}\right)=\left(\left\langle\alpha_{j} \mid \alpha_{j}\right\rangle M_{i j}\right)$ is symmetric with respect to transposition and its determinant is positive. Hence it can be diagonalized, so that $\Delta$ becomes a sum of second derivatives (with respect to new variables) with no mixed derivative terms.

\subsection{Rank two and three special cases}

Problems in solving the Neumann boundary value problem on $F$ is most often encountered in dimensions 2 and 3. We write down the explicit form of the Laplace operators in coordinates relative to the $\omega$-basis for ranks 2 and 3 derived from formula (10.3). For rank two the operator $\Delta$ is of the form

$$
\begin{array}{ll}
A_{2}:\left(\partial_{1}^{2}-\partial_{1} \partial_{2}+\partial_{2}^{2}\right) \phi=-\frac{4 \pi^{2}}{3}\left(a^{2}+a b+b^{2}\right) \phi, & F=\left\{0, \omega_{1}, \omega_{2}\right\}, \\
C_{2}:\left(2 \partial_{1}^{2}-2 \partial_{1} \partial_{2}+\partial_{2}^{2}\right) \phi=-2 \pi^{2}\left(a^{2}+4 a b+4 b^{2}\right) \phi, & F=\left\{0, \omega_{1}, \omega_{2}\right\}, \\
G_{2}:\left(\partial_{1}^{2}-3 \partial_{1} \partial_{2}+3 \partial_{2}^{2}\right) \phi=-\frac{4 \pi^{2}}{3}\left(3 a^{2}+3 a b+b^{2}\right) \phi, & F=\left\{0, \frac{\omega_{1}}{2}, \omega_{2}\right\} .
\end{array}
$$

Here, in order to simplify the notation, $\phi$ stands for $\phi_{\lambda}(x), \lambda=(a b)$ and $x=\left(\theta_{1} \theta_{2}\right)$. Although the same symbols are used for analogous objects in the three cases, their geometric meaning is very different. It is given by the appropriate matrix $M$ in (2.1). In particular, the vertices of $F$ form an equilateral triangle in case of $A_{2}$, for $C_{2}$ the triangle is half of a square, and it is a half of an equilateral triangle for $G_{2}$. In the semisimple case $A_{1} \times A_{1}$ one has $M=2\left(\begin{array}{ll}1 & 0 \\ 0 & 1\end{array}\right)$, therefore $\Delta=2 \partial_{1}^{2}+2 \partial_{2}^{2}$, and $\phi_{\lambda}(x)$ is the product of two orbit functions, one from each $A_{1}$. The fundamental domain is the square.

There are three 3 -dimensional cases to consider, namely $A_{3}, B_{3}$, and $C_{3}$. In addition there are four cases involving non-simple groups of the same rank. For $A_{3}, B_{3}$, and $C_{3}$ the result can be represented by the formulas

$$
\begin{aligned}
& A_{3}: \quad \Delta=\partial_{1}^{2}+\partial_{2}^{2}+\partial_{3}^{2}-\partial_{1} \partial_{2}-\partial_{2} \partial_{3}, \\
& B_{3}: \quad \Delta=\partial_{1}^{2}+\partial_{2}^{2}+2 \partial_{3}^{2}-\partial_{1} \partial_{2}-2 \partial_{2} \partial_{3}, \\
& C_{3}: \quad \Delta=2 \partial_{1}^{2}+2 \partial_{2}^{2}+\partial_{3}^{2}-2 \partial_{1} \partial_{2}-2 \partial_{2} \partial_{3} .
\end{aligned}
$$

\subsection{Orbit functions as eigenfunctions of other operators}

Orbit functions are eigenfunctions of many other operators. We consider examples of such operators.

With each $y \in E_{n}$ we associate the shift operator $T_{y}$ which acts on the exponential functions $e^{2 \pi \mathrm{i}\langle\lambda, x\rangle}$ as

$$
T_{y} e^{2 \pi \mathrm{i}\langle\lambda, x\rangle}=e^{2 \pi \mathrm{i}\langle\lambda, x+y\rangle}=e^{2 \pi \mathrm{i}\langle\lambda, y\rangle} e^{2 \pi \mathrm{i}\langle\lambda, x\rangle} .
$$

We define an action of elements of the Weyl group $W$ on functions, given on $E_{n}$, as $w f(x)=$ $f(w x)$. Now for each $y \in E_{n}$ we define an operator acting on orbit functions by the formula

$$
D_{y}=\sum_{w \in W} w T_{y} .
$$


Then

$$
\begin{aligned}
D_{y} \phi_{\lambda}(x) & =D_{y} \sum_{w \in W / W_{\lambda}} e^{2 \pi \mathrm{i}\langle w \lambda, x\rangle}=\sum_{w^{\prime} \in W} \sum_{w \in W / W_{\lambda}} e^{2 \pi \mathrm{i}\langle w \lambda, y\rangle} e^{2 \pi \mathrm{i}\left\langle w \lambda, w^{\prime} x\right\rangle} \\
& =\sum_{w \in W / W_{\lambda}} e^{2 \pi \mathrm{i}\langle w \lambda, y\rangle} \sum_{w^{\prime} \in W} e^{2 \pi \mathrm{i}\left\langle w \lambda, w^{\prime} x\right\rangle} \\
& =\sum_{w \in W / W_{\lambda}} e^{2 \pi \mathrm{i}\langle w \lambda, y\rangle} \sum_{w^{\prime} \in W} e^{2 \pi \mathrm{i}\left\langle w^{\prime-1} w \lambda, x\right\rangle} \\
& =\left|W_{\lambda}\right| \sum_{w \in W / W_{\lambda}} e^{2 \pi \mathrm{i}\langle w \lambda, y\rangle} \phi_{\lambda}(x)=\left|W_{\lambda}\right| \phi_{\lambda}(y) \phi_{\lambda}(x),
\end{aligned}
$$

that is, $\phi_{\lambda}(x)$ is an eigenfunction of the operator $D_{y}$ with eigenvalue $\left|W_{\lambda}\right| \phi_{\lambda}(y)$.

It is shown similarly that in the cases of $A_{n}, B_{n}, C_{n}, D_{n}$ orbit functions $\phi_{\lambda}(x)$ are eigenfunctions of the operators

$$
\sum_{w \in W} w \frac{\partial^{2}}{\partial^{2} x_{i}}, \quad i=1,2, \ldots, r,
$$

where $x_{1}, x_{2}, \ldots, x_{r}$ are orthogonal coordinates of the point $x, r=n+1$ for $A_{n}$ and $r=n$ for other cases. In fact, these operators are multiple to the Laplace operator $\Delta$.

It is easy to show that in the cases of $A_{n}, B_{n}, C_{n}$, and also $D_{n}$ with even $n$, orbit functions $\phi_{\lambda}(x)$ are solutions of the equations

$$
\sum_{w \in W} w \frac{\partial}{\partial x_{i}} f=0, \quad i=1,2, \ldots, r .
$$

\section{Orbit functions and symmetric polynomials}

\subsection{Orbit functions and monomial symmetric polynomials}

As is mentioned in Introduction, orbit functions are a certain modification of monomial symmetric polynomials $m_{\lambda}(y), \lambda \in P_{+}$. For simplicity, we restrict ourselves to the case of root systems and Weyl groups of $A_{n-1}, B_{n}, C_{n}$ and $D_{n}$. We use for $x \in E_{n}$ and for elements $\lambda \in P$ the orthogonal coordinate systems described in Section 3 (moreover we assume that orthogonal coordinates $m_{1}, m_{2}, \ldots, m_{n}$ of $\lambda \in P$ take only integral values in the cases of $A_{n-1}$ and $B_{n}$ ). Then elements of $W$ have a natural description in term of permutations and changes of signs.

In the expression for orbit functions $\phi_{\lambda}(x)=\sum_{\mu \in O(\lambda)} e^{2 \pi \mathrm{i} \sum_{i} x_{i} \mu_{i}}, \lambda \in P_{+}$, we replace each $e^{2 \pi \mathrm{i} x_{j}}$ by $y_{j}$. Then orbit functions $\phi_{\lambda}(x)$ turn into the Laurent polynomials (that is, polynomials in $\left.y_{1}, y_{2}, \ldots, y_{n}, y_{1}^{-1}, y_{2}^{-1}, \ldots, y_{n}^{-1}\right)$

$$
m_{\lambda}(y)=\sum_{\mu \in O(\lambda)} y^{\mu} \equiv \sum_{\mu \in O(\lambda)} y_{1}^{\mu_{1}} y_{2}^{\mu_{2}} \cdots y_{n}^{\mu_{n}}
$$

where $\mu_{1}, \mu_{2}, \ldots, \mu_{n}$ are orthogonal coordinates of $\mu \in P$. They are called monomial symmetric polynomials. They are very useful for studying symmetric (with respect to $W$ ) Laurent polynomials, which constitute orthogonal bases of the space $\mathbb{C}\left[y_{1}, y_{2}, \ldots, y_{n}\right]^{W}$ of all symmetric (under the Weyl group $W$ ) Laurent polynomials in $y_{1}, y_{2}, \ldots, y_{n}$ with respect to some scalar products.

For studying symmetric orthogonal polynomials in $\mathbb{C}\left[y_{1}, y_{2}, \ldots, y_{n}\right]^{W}$ one usually replaces $y^{\mu}$ by $e^{\mu}$, where $e^{\mu}$ is considered as a function on $E_{n}$ :

$$
e^{\mu}(x)=e^{\langle\mu, x\rangle}=e^{\mu_{1} x_{1}+\cdots+\mu_{n} x_{n}} .
$$


Then instead of the orbit functions $\phi_{\lambda}(x)$ considered above we obtain the modified orbit functions

$$
\tilde{\phi}_{\lambda}(x)=\sum_{\mu \in O(\lambda)} e^{\langle\mu, x\rangle}=\sum_{\mu \in O(\lambda)} e^{\mu_{1} x_{1}+\cdots+\mu_{n} x_{n}} .
$$

Usually in the theory of symmetric polynomials the functions (11.2) are denoted by $m_{\lambda}(x)$ (see, for example, [7]). We used the symbol $m_{\lambda}$ for polynomials (11.1). In order to be closer to the notations of the theory of symmetric polynomials, we denote functions (11.2), which are Laurent polynomials in $e^{x_{j}}, j=1,2, \ldots, n$, by $\hat{m}_{\lambda}(x)$. It is shown in the same way as in Section 9 that the functions $\hat{m}_{\lambda}(x)$ are eigenfunctions of the Laplace operator $\Delta=\frac{\partial^{2}}{\partial x_{1}^{2}}+\frac{\partial^{2}}{\partial x_{2}^{2}}+\cdots+\frac{\partial^{2}}{\partial x_{n}^{2}}$, namely,

$$
\Delta \hat{m}_{\lambda}(x)=\langle\lambda, \lambda\rangle \hat{m}_{\lambda}(x)=\left(\lambda_{1}^{2}+\lambda_{2}^{2}+\cdots+\lambda_{n}^{2}\right) \hat{m}_{\lambda}(x) .
$$

As a rule, different types of orthogonal symmetric Laurent polynomials in $e^{x_{j}}, j=1,2, \ldots, n$, are eigenfunctions of operators, which are obtained from $\Delta$ by adding some terms. We shall see this below. We shall also see how monomial symmetric polynomials $\hat{m}_{\lambda}$ are used for construction of such eigenfunctions.

Note that if we take integral orthogonal coordinates $m_{1}, m_{2}, \ldots, m_{n}$ in the $A_{n}$ case in such a way that $m_{1} \geq m_{2} \geq \cdots \geq m_{n} \geq 0$, then Laurent polynomials $m_{\lambda}(y)$ and $\hat{m}_{\lambda}(x)$ turn into usual (not Laurent) polynomials.

\subsection{Jacobi symmetric polynomials}

Jacobi polynomials in one variable are well-known orthogonal polynomials of the theory of special functions of mathematical physics. Jacobi polynomials of many variables are symmetric (Laurent) polynomials which are defined by means of polynomials $\hat{m}_{\lambda}, \lambda \in P_{+}$. We fix for every root $\alpha \in R$ a positive integer $k_{\alpha}$ such that $k_{w \alpha}=k_{\alpha}$ for each $w \in W$. Since there exist only one or two $W$-orbits of roots in $R$, we have one or two numbers $k$, respectively. We introduce the notation

$$
\rho_{k}=\frac{1}{2} \sum_{\alpha \in R} k_{\alpha} \alpha .
$$

Next we construct the operator

$$
M_{2}=\Delta-\sum_{\alpha \in R} k_{\alpha} \frac{1+e^{\alpha}}{1-e^{\alpha}} \partial_{\alpha},
$$

where $\partial_{\alpha}$ is the derivative in direction of the root $\alpha$ and $e^{\alpha}$ is the function on $E_{n}$, defined in the previous subsection. The following properties of the operator $M_{2}$ are proved in [39, 40]:

(i) $M_{2}$ preserves the space $\mathbb{C}\left[e^{x_{1}}, e^{x_{2}}, \ldots, e^{x_{n}}\right]^{W}$.

(ii) The action of $M_{2}$ on functions $\hat{m}_{\lambda}(x)$ is triangular:

$$
M_{2} \hat{m}_{\lambda}(x)=\left\langle\lambda, \lambda+2 \rho_{k}\right\rangle \hat{m}_{\lambda}(x)+\sum_{\mu<\lambda} \hat{m}_{\mu}(x)
$$

where, as before, $\mu<\lambda$ means that $\lambda-\mu \in Q_{+}$and $\mu \neq \lambda$.

The following theorem is crucial in the definition of Jacobi symmetric polynomials, proof of which can be found in [39] and [40]. 
Theorem 2. To every $\lambda \in P_{+}$there corresponds a unique polynomial $J_{\lambda} \in \mathbb{C}\left[e^{x_{1}}, \ldots, e^{x_{n}}\right]^{W}$ such that

$$
\begin{aligned}
& J_{\lambda}(x)=\hat{m}_{\lambda}(x)+\text { lower order terms, } \\
& M_{2} J_{\lambda}(x)=\left\langle\lambda, \lambda+2 \rho_{k}\right\rangle J_{\lambda}(x)
\end{aligned}
$$

where under lower order terms linear combinations of functions $\hat{m}_{\mu}(x), \mu \in P_{+}$, with $\mu<\lambda$ are understood.

Replacing $e^{x_{j}}$ by $y_{j}, j=1,2, \ldots, n$, in $J_{\lambda}$ we obtain symmetric (Laurent) polynomials which are called Jacobi polynomials of many variables. They are orthogonal with respect to a certain positive measure which will be given in the next subsection.

Replacing $y_{j}$ by $e^{2 \pi \mathrm{i} x_{j}}, j=1,2, \ldots, n$, in Jacobi polynomials we obtain functions of $x_{1}, x_{2}, \ldots$, $x_{n}$ which are linear combinations of orbit functions and, therefore, are invariant with respect to the affine Weyl group $W^{\text {aff }}$. This means that, as in the case of orbit functions, they are uniquely determined by their values on the fundamental domain of the group $W^{\text {aff }}$.

\subsection{Macdonald symmetric polynomials}

Macdonald symmetric (Laurent) polynomials are also constructed by means of monomial symmetric polynomials. They are a quantum analogue of Jacobi symmetric polynomials, considered in the previous subsection.

We introduce a variable $q$ and with every root $\alpha \in R$ associate a variable $t_{\alpha}$ such that $t_{\alpha}=t_{w \alpha}, w \in W$ (therefore, there exist one or two variables $\left.t\right)$. Let $\mathbb{C}_{q, t_{\alpha}} \equiv \mathbb{C}\left(q, t_{\alpha}\right)$ be the field of rational functions in $q$ and $t_{\alpha}$. If $e^{x_{1}}, e^{x_{2}}, \ldots, e^{x_{n}}$ are such as in the previous subsection, then

$$
\mathbb{C}_{q, t_{\alpha}}\left[e^{x_{1}}, e^{x_{2}}, \ldots, e^{x_{n}}\right]
$$

will denote the set of (Laurent) polynomials in $e^{x_{1}}, e^{x_{2}}, \ldots, e^{x_{n}}$ with coefficients from $\mathbb{C}\left(q, t_{\alpha}\right)$.

For each function $\sum_{\lambda} a_{\lambda} e^{\lambda}$ on $E_{n}$ we define the constant term $\left[\sum_{\lambda} a_{\lambda} e^{\lambda}\right]_{0}$ coinciding with

$$
\left[\sum_{\lambda} a_{\lambda} e^{\lambda}\right]_{0}=a_{0}
$$

Now one can determine an inner product $\langle\cdot, \cdot\rangle_{q, t_{\alpha}}$ on $\mathbb{C}_{q, t_{\alpha}}\left[e^{x_{1}}, e^{x_{2}}, \ldots, e^{x_{n}}\right]$ by the formula

$$
\left\langle p_{1}, p_{2}\right\rangle_{q, t_{\alpha}}=|W|^{-1}\left[p_{1} \overline{p_{2}} \Delta_{q, t_{\alpha}}\right]_{0},
$$

where the bar over $p_{2}$ denotes the linear involution which is uniquely determined by $\overline{e^{\lambda}}=e^{-\lambda}$, and

$$
\Delta_{q, t_{\alpha}}=\prod_{\alpha \in R} \prod_{i=1}^{\infty} \frac{1-q^{2 i} e^{\alpha}}{1-t_{\alpha}^{2} q^{2 i} e^{\alpha}} .
$$

Here $\Delta_{q, t_{\alpha}}$ must be considered as a Laurent series in $q$ and $t_{\alpha}$ with coefficients from the space $\mathbb{C}\left[e^{x_{1}}, e^{x_{2}}, \ldots, e^{x_{n}}\right]$. The inner product (11.5) is non-degenerate and invariant with respect to $W$. I. Macdonald [7] proved the following theorem:

Theorem 3. There exists a unique family $P_{\lambda} \in \mathbb{C}_{q, t_{\alpha}}\left[e^{x_{1}}, e^{x_{2}}, \ldots, e^{x_{n}}\right]^{W}, \lambda \in P_{+}$, satisfying the conditions

$$
\begin{aligned}
& P_{\lambda}=\hat{m}_{\lambda}+\sum_{\mu<\lambda} a_{\lambda}^{\mu} \hat{m}_{\mu}, \quad a_{\lambda}^{\mu} \in \mathbb{C}_{q, t_{\alpha}}, \\
& \left\langle P_{\lambda}, P_{\mu}\right\rangle_{q, t_{\alpha}}=0, \quad \text { if } \quad \lambda \neq \mu \text {. }
\end{aligned}
$$


Replacing $e^{x_{j}}$ by $y_{j}, j=1,2, \ldots, n$, in $P_{\lambda}$ we obtain (for each fixed values of $q$ and $t_{\alpha}$ ) orthogonal symmetric polynomials which are called Macdonald symmetric polynomials.

Replacing $y_{j}$ by $e^{2 \pi \mathrm{i} x_{j}}, j=1,2, \ldots, n$, in Macdonald polynomials we obtain orthogonal functions which are finite linear combinations of orbit functions and are invariant with respect to the affine Weyl group $W^{\text {aff }}$. This means that, as in the case of orbit functions, they are uniquely determined by their values on the fundamental domain of $W^{\text {aff }}$.

For some special values of $q$ and $t_{\alpha}$ (see [7]) Macdonald polynomials reduce to more simple sets of polynomials:

(a) If $t_{\alpha}=1$, then $P_{\lambda}=\hat{m}_{\lambda}$ independently of $q$.

(b) If $t_{\alpha}=q$ for all $\alpha \in R$, then $P_{\lambda}=\chi_{\lambda}$, where $\chi_{\lambda}$ are characters of finite dimensional irreducible representations of the corresponding simple Lie groups.

(c) If $q, t_{\alpha} \rightarrow 1$ in such a way that $t_{\alpha}=q^{k_{\alpha}}, k_{\alpha} \in \mathbb{Z}_{+}$are fixed, then $P_{\lambda} \rightarrow J_{\lambda}$, where $J_{\lambda}$ are Jacobi symmetric polynomials. In this case the inner product for Macdonald polynomials turns into the scalar product

$$
\left\langle p_{1}, p_{2}\right\rangle=|W|^{-1}\left[p_{1} \overline{p_{2}} \delta^{k} \overline{\delta^{k}}\right]_{0}
$$

with respect to which Jacobi polynomials are orthogonal. Here $\delta^{k}=\prod_{\alpha \in R_{+}}\left(e^{\alpha / 2}-e^{-\alpha / 2}\right)^{k_{\alpha}}$.

(d) If $q=0$ and $t_{\alpha}=1 / p$, where $p$ is a prime integer, then $P_{\lambda}$ are zonal spherical polynomials for the corresponding $p$-adic group.

(e) For the root system $A_{1}$, Macdonald polynomials reduce to continuous $q$-ultraspherical orthogonal polynomials of one variable (for definition and properties of these polynomials see [41]).

\section{Acknowledgements}

The first author (AK) acknowledges CRM of University of Montreal for hospitality when this paper was under preparation. We are grateful for partial support for this work to the National Research Council of Canada and to MITACS.

[1] Patera J., Orbit functions of compact semisimple Lie groups as special functions, in Proceedinds of Fifth International Conference "Symmetry in Nonlinear Mathematical Physics" (June 23-29, 2003, Kyiv), Editors A.G. Nikitin, V.M. Boyko, R.O. Popovych and I.A. Yehorchenko, Proceedings of Institute of Mathematics, Kyiv, 2004, V.50, Part 3, 1152-1160.

[2] Vilenkin N.Ja., Klimyk A.U., Representations of Lie groups and special functions, Vols. 1-3, Dordrecht, Kluwer, 1991-1993.

[3] Miller W., Lie theory and special functions, New York, Academic Press, 1968.

[4] Vilenkin N.Ja., Special functions and the theory of group representations, Providence RI, Amer. Math. Soc., 1968.

[5] Macdonald I.G., Symmetric functions and Hall polynomials, 2nd ed., Oxford, Oxford Univ. Press, 1995.

[6] Macdonald I.G., A new class of symmetric functions, Publ. I.R.M.A. Strasbourg, 372/S-20, Actes 20, 1988, Séminaire Lotharingien, 131-171.

[7] Macdonald I.G., Orthogonal polynomials associated with root systems, Séminaire Lotharingien de Combinatoire, Actes B45a, Strasbourg, 2000.

[8] Vilenkin N.Ja., Klimyk A.U., Representations of Lie groups and special functions: recent advances, Dordrecht, Kluwer, 1995.

[9] Moody R.V., Patera J., Computation of character decompositions of class functions on compact semisimple Lie groups, Math. Comp., 1987, V.48, 799-827.

[10] Moody R.V., Patera J., Elements of finite order in Lie groups and their applications, XIII Int. Colloq. on Group Theoretical Methods in Physics, Editor W. Zachary, Singapore, World Scientific Publishers, 1984, 308-318.

[11] McKay W.G., Moody R.V., Patera J., Tables of $E_{8}$ characters and decomposition of plethysms, in Lie Algebras and Related Topics, Editors D.J. Britten, F.W. Lemire and R.V. Moody, Providence R.I., Amer. Math. Society, 1985, 227-264. 
[12] McKay W.G., Moody R.V., Patera J., Decomposition of tensor products of $E_{8}$ representations, Algebras Groups Geom., 1986, V.3, 286-328.

[13] Patera J., Sharp R.T., Branching rules for representations of simple Lie algebras through Weyl group orbit reduction, J. Phys. A: Math. Gen., 1989, V.22, 2329-2340.

[14] Grimm S., Patera J., Decomposition of tensor products of the fundamental representations of $E_{8}$, $C R M$ Proc. Lecture Notes, 1997, V.11, 329-355.

[15] Atoyan A., Patera J., Properties of continuous Fourier extension of the discrete cosine transform and its multidimensional generalization, J. Math. Phys., 2004, V.45, 2468-2491; math-ph/0309039.

[16] Rao K.R., Yip P., Discrete cosine transform — algorithms, advantages, applications, New York, Academic Press, 1990.

[17] Kane R., Reflection groups and invariants, New York, Springer, 2002.

[18] Humphreys J.E., Reflection groups and Coxeter groups, Cambridge, Cambridge Univ. Press, 1990.

[19] Humphreys J.E., Introduction to Lie Algebras and Representation Theory, New York, Springer, 1972.

[20] Pinsky M.A., The Eigenvalues of an Equilateral Triangle, SIAM J. Math. Anal., 1980, V. 11, 819-827.

[21] Patera J., Algebraic solution of the Neumann boundary problems on fundamental regions of a compact semisimple Lie group, CRM Preprint, Montreal, 2003.

[22] Patera J., Compact simple Lie groups and their $C$-, $S$-, and E-transforms, SIGMA, 2005, V.1, paper 025, 6 pages; math-ph/0512029.

[23] Bremner M.R., Moody R.V., Patera J., Tables of dominant weight multiplicities for representations of simple Lie algebras, New York, Marcel Dekker, 1985.

[24] McKay W.G., Patera J., Rand D.W., Tables of representations of simple Lie algebras, Montreal, CRM, 1990.

[25] Champagne B., Kjiri M., Patera J., Sharp R.T., Description of reflection generated polytopes using decorated Coxeter diagrams, Can. J. Phys., 1995, V.73, 566-584.

[26] Moody R.V., Patera J., Voronoi and Delaunay cells of root lattices: classification of their faces and facets by Coxeter-Dynkin diagrams, J. Phys. A: Math. Gen., 1992, V.25, 5089-5134.

[27] McKay W.G., Patera J., Sannikoff D., The computation of branching rules for representations of semisimple Lie algebras, in Computers in Nonassociative Rings and Algebras, Editors R.E. Beck and B. Kolman, New York, Academic Press, 1977, 235-278.

[28] Gingras F., Patera J., Sharp R.T., Orbit-orbit branching rules between simple low-rank algebras and equalrank subalgebras, J. Math. Phys., 1992, V.33, 1618-1626.

[29] Patera J., Sharp R.T., Branching rules for representations of simple Lie algebras through Weyl group orbits reduction, J. Phys. A: Math. Gen., 1989, V.22, 2329-2340.

[30] Patera J., Zaratsyan A., Discrete and continuous cosine transform generalized to Lie groups $S U(2) \times S U(2)$ and $O(5)$, J. Math. Phys., 2005, V.46, 053514, 25 pages.

[31] Patera J., Zaratsyan A., Discrete and continuous cosine transform generalized to Lie groups $S U(2)$ and $G_{2}$, J. Math. Phys., 2005, V.46, 113506, 17 pages.

[32] Lemire F.W., Patera J., Congruence number, a generalisation of SU(3) triality, J. Math. Phys., 1980, V.21, 2026-2027.

[33] Zhelobenko D.P., Compact Lie groups and their representations, Moscow, Nauka, 1970.

[34] McKay W.G., Patera J., Tables of dimensions, indices and branching rules for representations of simple Lie algebras, New York, Marcel Dekker, 1981.

[35] Strang G., The discrete cosine transform, SIAM Review, 1999, V.41, 135-147.

[36] Kac V., Automorphisms of finite order of semisimple Lie algebras, J. Funct. Anal. Appl., 1969, V.3, 252-255.

[37] Moody R.V., Patera J., Characters of elements of finite order in simple Lie groups, SIAM J. Algebraic Discrete Methods, 1984, V.5, 359-383.

[38] McKay W.G., Moody R.V., Patera J., Pianzola A., The 785 conjugacy classes of rational elements of finite

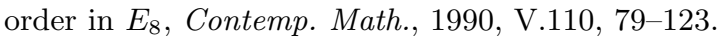

[39] Heckman G.J., Opdam E.M., Root systems and hypergeometric functions. I, Compos. Math., 1987, V.64, 329-352.

[40] Heckman G.J., Root systems and hypergeometric functions. II, Compos. Math., 1987, V.64, 353-373.

[41] Gasper G., Rahman M., Basic hypergeometric functions, Cambridge, Cambridge Univ. Press, 1990. 